\title{
Categorization of Boranes Into Clan Series
}

\author{
Enos Masheija Rwantale Kiremire ${ }^{1}$, Ivan Lule ${ }^{1}$ \\ ${ }^{1}$ National Planning Authority, Plot 17B $2^{\text {nd }}$ Floor, Clement Hill Road, P. O. Box 21434, Kampala, Uganda. \\ Correspondence: Enos Masheija Rwantale Kiremire, National Planning Authority, Plot 17B $2^{\text {nd }}$ Floor, Clement Hill Road, \\ P. O. Box 21434, Kampala, Uganda. E-mail: emkiremire@gmail.com
}

Received: May 21, 2019 Accepted: Feb. 17, 2020 Online Published: Apr. 3, 2020

doi:10.5539/ijc.v12n1p107

URL: https://doi.org/10.5539/ijc.v12n1p107

\begin{abstract}
Boranes, despite their instability in nature, can be regarded as hydrocarbon relatives since a $[\mathrm{BH}]$ fragment corresponds to a carbon $[\mathrm{C}]$ skeletal element in terms of the number of valence electrons. The borane formula which can be expressed as $\mathrm{B}_{\mathrm{n}} \mathrm{H}_{\mathrm{m}}$ usually appears in such a way that when (n) is even, then (m) is even and when (n) is odd, (m) is odd as well. Through the study of cluster series, it appears that the cluster number $\mathrm{K}$ which represents skeletal linkages is usually a whole number. This inherent characteristic confers unique order within borane clusters with nodal connectivity of 5 and the polyhedral nature of the borane clusters. The orderliness of the borane clusters is reflected by the ease of their categorization into clan series and their readily constructed geometrical isomeric structures. The cluster valence electrons can easily be calculated using one of the six recently discovered fundamental equations.
\end{abstract}

Keywords: isomeric structures, polyhedral triangles, valence equations, clusters, boranes, skeletal nodes

\section{Introduction}

Borane hydride clusters have fascinated and intrigued scientists since their discovery in 1912 due to their unique characteristic features of polyhedral shapes, apparent strange stoichiometries and bonding (Stock, 1933; Lipscomb, 1963; Wade, 1971, 1976; Jemmis, et al, 2001a-b; Mingos,1984, Kiremire, 2014). Furthermore, some applications of borane clusters have been discovered and the field is expanding (Hawthorne, et al, 1990). Arising from their polyhedral shapes, certain names such as closo, nido, arachno, hypho and klapo (Housecroft and Sharpe, 2005; Meissler, et al, 2014) were associated with characteristic shapes. In addition, attempts to explain their structures have given rise to concepts such as Wade-Mingos rules (Mingos, 1972; Welch, 2013), Jemmis mno rules (Jemmis, et al, 2001b), styx numbers (Lipscomb, 1963), and topological theories (Teo, et al,1984), among others. Closer analysis of clusters revealed that the skeletal elements and clusters from the main group and transition elements can be analyzed and categorized more extensively using the $4 \mathrm{~N}$ series method and that they strictly obey the law of skeletal numbers and their valences (Kiremire, 2017ab). In this paper, selected sample of boranes will be categorized according to their CLAN SERIES, $\mathrm{D}^{\mathrm{z}}$ where $\mathrm{D}^{\mathrm{z}}$ originates from the categorization cluster parameter $\mathrm{K}^{*}=\mathrm{C}^{\mathrm{y}}+\mathrm{D}^{\mathrm{z}}$ (Kiremire, 2019a-c), the $\mathrm{K}(\mathrm{n}$ ) parameter will be utilized to construct the polyhedral isomers of borane clusters and derive their cluster formulas and valence electrons. The skeletal numbers of elements and ligands discovered and utilized earlier are provided in appendix 1 and 2 for ease of reference and recognition as the concept is still new (Kiremire, 2017a).

\section{Results and Discussion}

\subsection{The Skeletal Numbers and Skeletal Linkages}

The skeletal elements of the periodic table particularly the main group and transition metals do possess intrinsic skeletal numbers $(\mathrm{K})$ (Kiremire, 2016). These simply indicate the number of electron pairs a given skeletal element needs for it to achieve the 8-electron configuration (octet rule) for main group elements or the 18-electron configuration in the case of transition elements(2017c). When the skeletal number is doubled, then we get the number of skeletal linkages that can be attached to the element or the number of single electron donation the element requires to attain the respective 8 or 18 electron-rule. In the case of boron, $\mathrm{K}=2.5$ which means it requires 2.5 electron pairs $(\mathrm{V}=2 \times 2.5=5 \mathrm{e})$ so as to achieve the octet rule $=3+5=8$. Thus, since the boron atom has 3 electrons, when it receives 5 others, it achieves the noble gas configuration of 8 . In principle, the boron skeletal element can be regarded as portraying 5 skeletal linkages in its cluster compounds. The skeletal numbers of other elements can be found in published articles (Kiremire 2019a-c). 


\subsection{Skeletal Elements as Nodes and Boron Exerting a Skeletal Valence of Five}

In the case of boranes, we can regard the boron skeletal element as a node which can have 5 linkages around it since it has a skeletal number $\mathrm{K}=2.5$ and $\mathrm{V}=2 \mathrm{~K}=5$ ). We know that a hydrogen element carries one electron and hence it is regarded as a single electron donor or ligand. In principle, we expect a boron skeletal element to form a simple mono-skeletal cluster with the formula $\mathrm{BH}_{5}$. But what is normally encountered is the cluster $\mathrm{BH}_{4}{ }^{-}$. According to the $4 \mathrm{~N}$ series approach, $(-1)$ charge is equivalent to a single $\mathrm{H}$ element. Therefore, we can consider $\mathrm{BH}_{4}{ }^{-}$as being derived from $\mathrm{BH}_{5}$ by replacing $(\mathrm{H})$ with $(-1)$ charge. If the replacement process is continued, we end up with the ion $\mathrm{B}^{5-}$. The possible species that can be generated are shown in Figure 1. According to the $4 \mathrm{~N}$ series approach all the generated species are equivalent. That is, $\mathrm{BH}_{5} \equiv \mathrm{BH}_{4}{ }^{-} \equiv \mathrm{BH}_{3}{ }^{2-} \equiv \mathrm{BH}_{2}{ }^{3-} \equiv \mathrm{BH}^{4-} \equiv \mathrm{B}^{5-}$. When the cluster has two or more boron skeletal elements or NODES, each of the nodes will exert 5 linkages. This is shown in Figures 2 for $\mathrm{B}_{2} \mathrm{H}_{8}$ and its isomers and 3 for $\mathrm{B}_{2} \mathrm{H}_{6}$ and its bridged isomer. The $\mathrm{B}_{2} \mathrm{H}_{7}-$ was identified and an IR studies conducted (Matsui \& Taylor, 989). The diborane molecule $\mathrm{B}_{2} \mathrm{H}_{6}$ is well known and has two bridging $\mathrm{H}$ ligands. The bridging structure can be viewed as a rearrangement of the 5-coordinated skeletal nodes. More than 60 isomeric graphical structures have been sketched according to the skeletal valence $(\mathrm{V}=5=$ five skeletal or nodal linkages) of boron. Clearly, the concept of skeletal number and valence make a lot of sense in rationalizing the cluster formulas of many complexes.

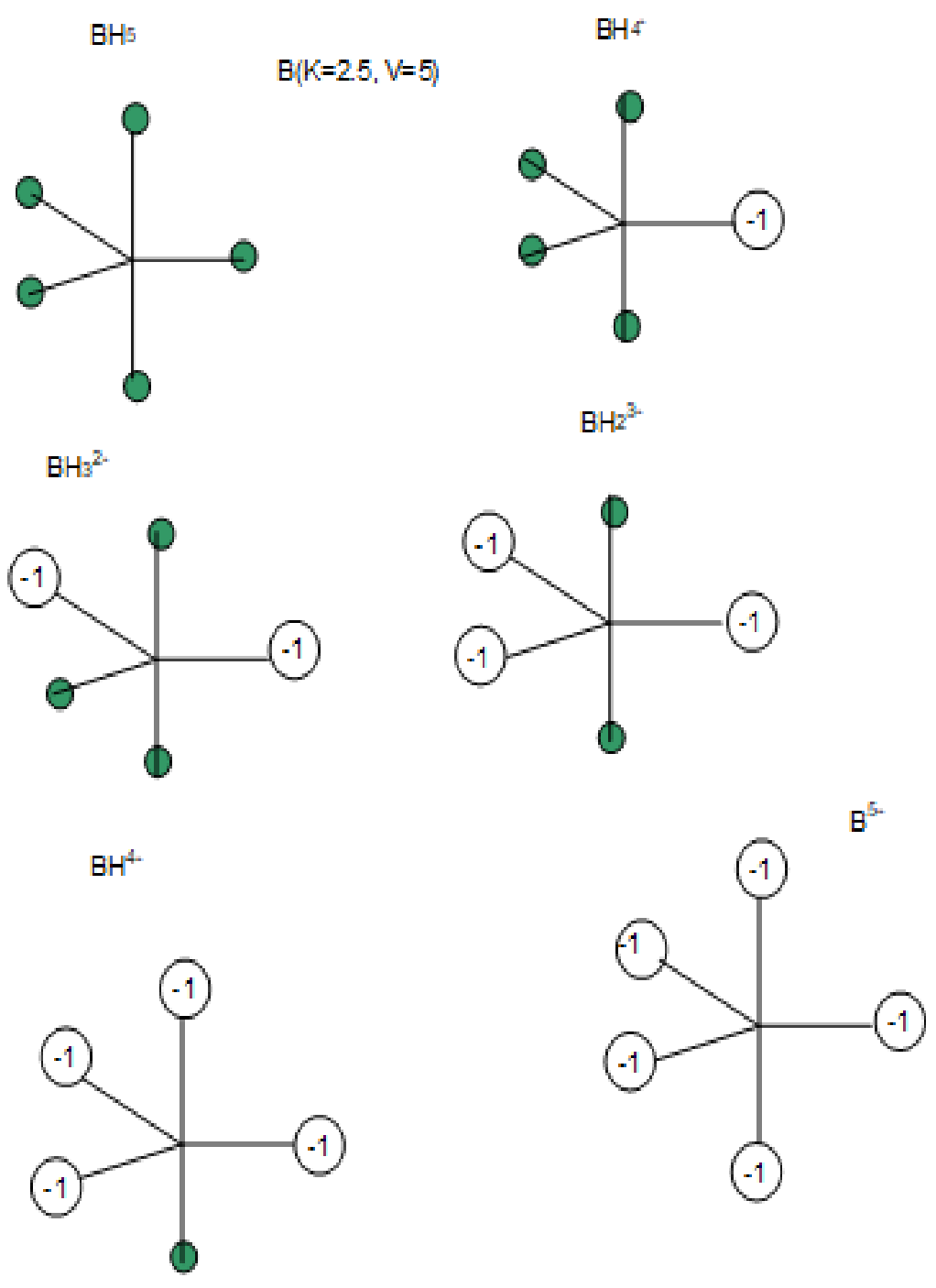

Figure 1. Equivalent isomeric structures of boron skeletal element 
$\mathrm{EX}-1 . \mathrm{B}_{2} \mathrm{H}_{8}$

$\mathrm{K}=2[2.5]-8[0.5]=1, \mathrm{n}=2$

$\mathrm{K}(\mathrm{n})=1(2)$

2[2]-1=3

$S=4 n+6$

$\mathrm{K}=2 \mathrm{n}-3$

$\mathrm{Kp}=\mathrm{C}^{-2} \mathrm{C}[\mathrm{M} 4]$

$\mathrm{K}^{\star}=\mathrm{C}^{-2}+\mathrm{D}^{4}$

$\mathrm{y}=-2, \mathrm{z}=4$

$\mathrm{VE} 0=2 \mathrm{Z}+2=2[4]+2=10$

$\mathrm{VE}=\mathrm{VE} 0+2 \mathrm{n}=10+2[2]=14$

$\mathrm{VF}=2[3]+8=14$

VED $z=4 z+2=4[4]+2=18$

$\mathrm{VE}=\mathrm{VED} z+2 \mathrm{y}=18+2[-2]=14$

$\mathrm{VE}=2 \mathrm{y}+4 \mathrm{z}+2=2[-2]+4[4]+2=14$

$\mathrm{VE}=8 \mathrm{n}-2 \mathrm{~K}=8[2]-2[1]=14$

$\mathrm{VE}=4 n+6=4[2]+6=14$

$\mathrm{VE}=\mathrm{VE} 0+2 \mathrm{y}+2 \mathrm{z}=10+2[-2]+2[4]=14$

$\mathrm{B}_{2} \mathrm{H}_{8}: \mathrm{K}=1$, the 2 boron skeletal elements are linked by one bond.

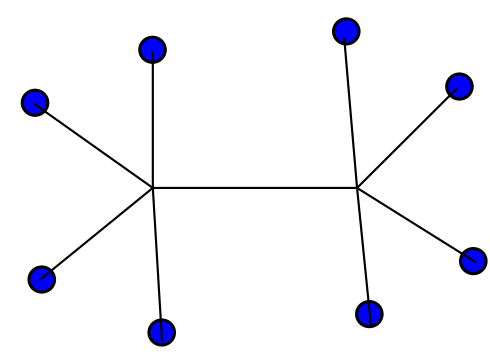

Figure 2A. Isomeric graphical structure of $\mathrm{B}_{2} \mathrm{H}_{8}$
$\mathrm{B}(\mathrm{K}=2.5, \mathrm{~V}=2 \mathrm{~K}=5$; Boron skeletal element has 5 skeletal linkages)

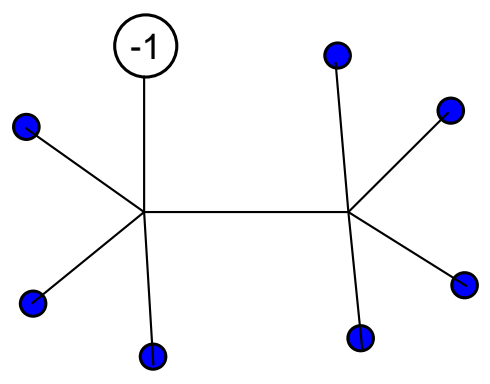

Figure 2B. Isomeric graphical structure of $\mathrm{B}_{2} \mathrm{H}_{7}$

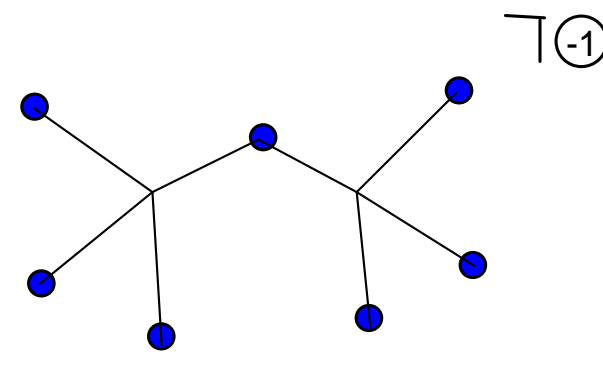

Figure 2C. Rearranement of 2B

Boron skeletal element strictly conforms to a skeletal valence of 5 

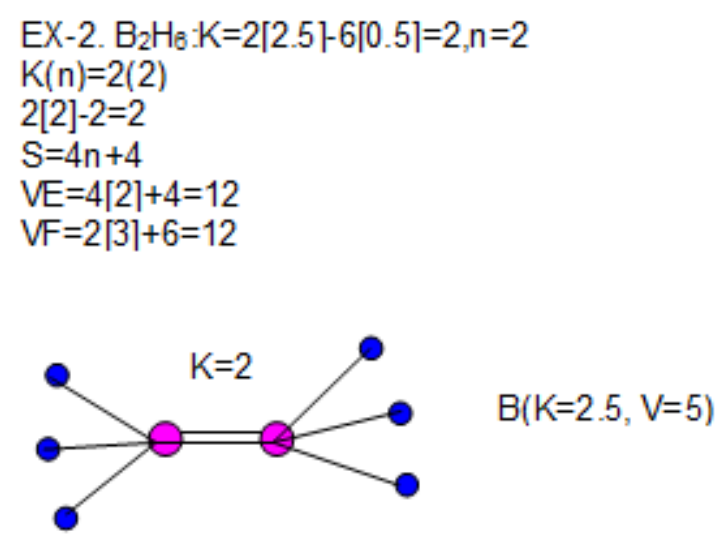

Figure 3. Isomeric graphical structure of $\mathrm{B}_{2} \mathrm{H}_{6}$

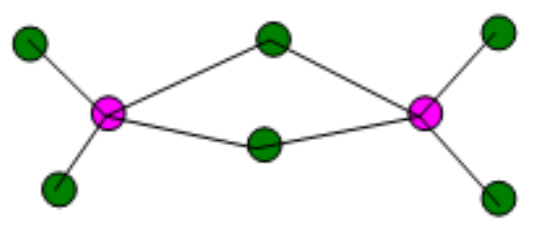

Figure 4. Rarrangement of Figure 3

\subsection{The Six Equations for Calculating the Cluster Valence Electrons}

Some or all the six equations have been used in the analysis of many clusters (Kiremire, 2019b) are being applied here in the analysis of the selected borane clusters. They all give the same result of the cluster valence electrons (VE) as the one calculated from the cluster formula (VF). This underpins the validity of the developed cluster valence equations. They are derived from the series equations and the capping principle of the $4 \mathrm{~N}$ series method. These equations are:

i. $\quad \mathrm{K}(\mathrm{n}) ; \mathrm{VE}=8 \mathrm{n}-2 \mathrm{~K}$

ii. $\mathrm{S}=4 \mathrm{n}+\mathrm{q} ; \mathrm{VE}=4 \mathrm{n}+\mathrm{q}$

$\mathrm{K}=\mathrm{C}^{\mathrm{y}}+\mathrm{D}^{\mathrm{z}} ; \mathrm{VE} 0=2 \mathrm{z}+2, \mathrm{VEDz}=4 \mathrm{z}+2, \mathrm{y}+\mathrm{z}=\mathrm{n}=$ the number of skeletal elements.

iii. $\mathrm{VE}=\mathrm{VE} 0+2 \mathrm{n}$

iv. $V E=V E D z+2 y$

v. $\mathrm{VE}=2 \mathrm{y}+4 \mathrm{z}+2$

vi. $V E=V E 0+2 y+2 z$

The symbol $n=$ the number of skeletal elements in a cluster excluding the ligands, $\mathrm{K}=$ skeletal linkages linking up the skeletal elements, $\mathrm{S}=$ series equation, $\mathrm{q}$ is a numerical variable that defines the type of the cluster, the $\mathrm{K}$ value can also be expressed in terms of $\mathrm{C}^{\mathrm{y}}+\mathrm{D}^{\mathrm{z}}$ where $\mathrm{C}$ and $\mathrm{D}$ represent the categorization of a cluster, $\mathrm{y}$ is in principle, the number of the capping skeletal elements, and $\mathrm{z}$ is the number of the nuclear elements.

Some of these or all the equations will be applied in the analysis of the following cluster examples. 

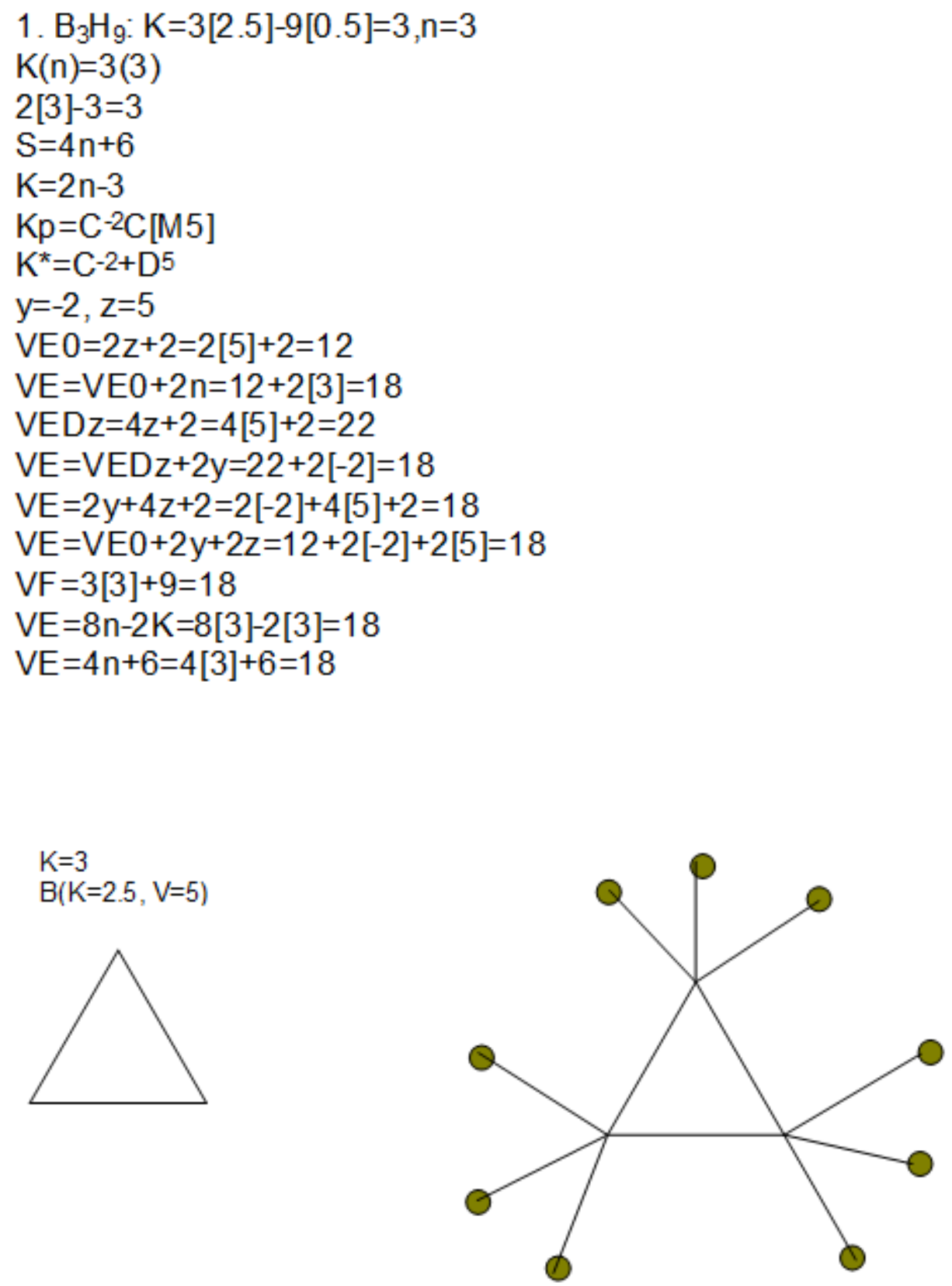

Figure 5. Isomeric graphical structure of $\mathrm{B}_{3} \mathrm{H}_{9}$

The cluster categorization is derived from the cluster series equation. The series equation is readily obtained from the following relationships.

$$
\begin{gathered}
\mathrm{S}=4 \mathrm{n}+\mathrm{q}=\mathrm{VE} \\
\mathrm{VE}=8 \mathrm{n}-2 \mathrm{~K}
\end{gathered}
$$

Another relationship is readily derived from these two equations as follows:

$$
\begin{gathered}
4 n+q=8 n-2 K \\
q=4 n-2 K \\
q / 2=2 n-K
\end{gathered}
$$

Knowing the values of $\mathrm{n}$ and $\mathrm{K}$ of a cluster, the $\mathrm{q}$ value of a cluster can be derived from (V). Hence the cluster series equation can readily be obtained. From the series equation, the $\mathrm{K}$ value is then expressed in terms of $\mathrm{n}$ and $\mathrm{q}$. The $\mathrm{Kp}$ value and $\mathrm{K}^{*}$ can also be derived from $\mathrm{K}$. The $\mathrm{K}^{*}$ is finally expressed in terms of $\mathrm{C}^{\mathrm{y}}$ and $\mathrm{D}^{\mathrm{z}}$ which determine the categorization of a cluster. This approach has been applied in the example 1 given above. 

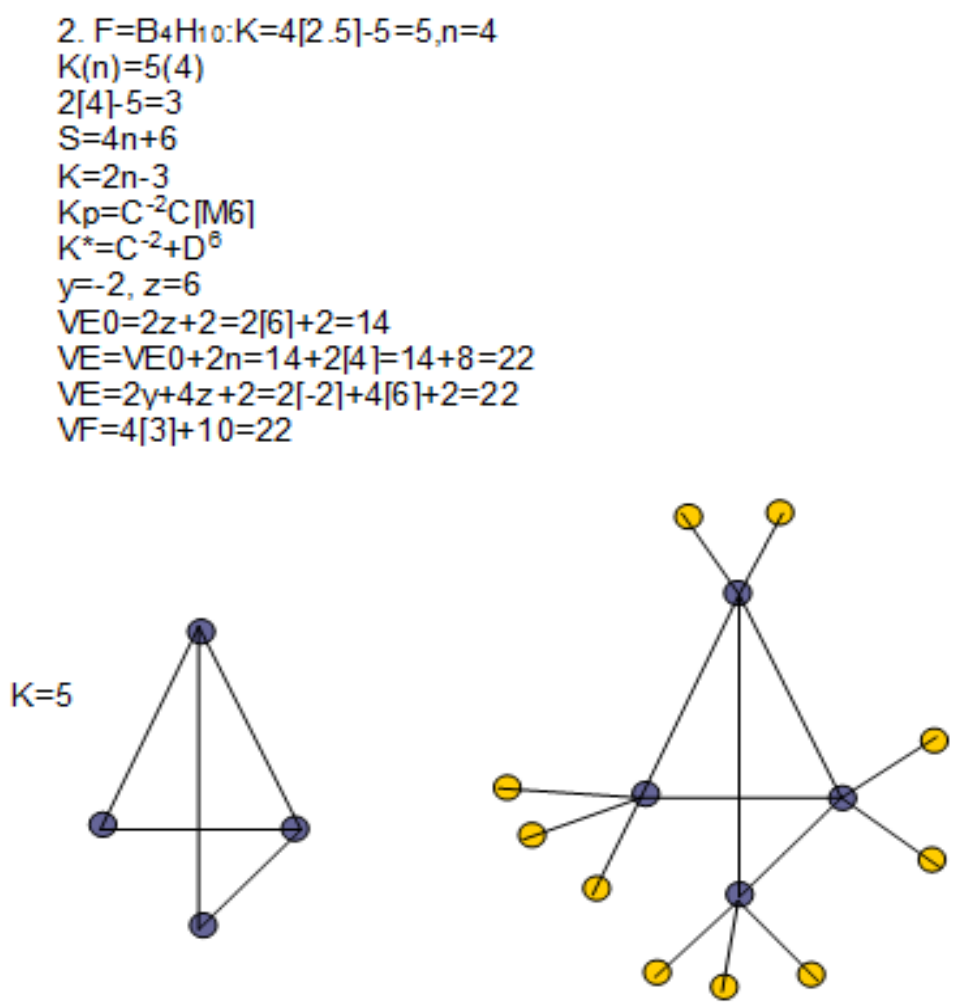

Figure 6. Isomeric graphical structure of $\mathrm{B}_{4} \mathrm{H}_{10}$

3. $\mathrm{F}=\mathrm{B}_{9} \mathrm{H}_{13}: \mathrm{K}=9[2.5+-13[0.5]=16, \mathrm{n}=9$

$\mathrm{K}(\mathrm{n})=16(9)$

2 [9]-16=2

$\mathrm{S}=4 \mathrm{n}+4$

$\mathrm{K}=2 \mathrm{n}-2$

$\mathrm{Kp}=\mathrm{C}^{-1} \mathrm{C}$ [M101

$\mathrm{K}^{*}=\mathrm{C}^{-1}+\mathrm{D}^{10}$

$\mathrm{y}=-1, \mathrm{z}=10$

VE $0=2 z+2=2[10]+2=22$

$\mathrm{VE}=\mathrm{VE} 0+2 \mathrm{n}=22+2[9]=40$

$\mathrm{VE}=\mathrm{VE} 0+2 \mathrm{y}+2 \mathrm{z}=22+2[-1]+2[10]=40$

$\mathrm{VE}=2 \mathrm{y}+4 \mathrm{z}+2=2[-1]+4[10]+2=40$

$\mathrm{VF}=9\lceil 3\rceil+13=40$

$\mathrm{VE}=8 n-2 \mathrm{~K}=8[9]-2\lceil 16]=40$

$\mathrm{VE}=4 \mathrm{n}+4=4[9]+4=40$

VED $z=4 z+2=4[10\rceil+2=42$

$\mathrm{VE}=\mathrm{VED} z+2 \mathrm{y}=42+2[-1]=40$

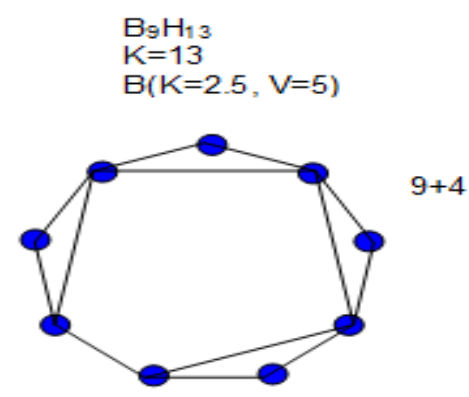

$K(n)=13(9): 13=9+4$

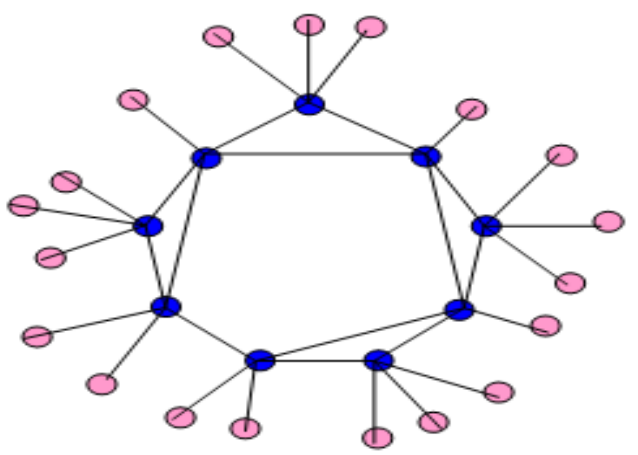

Figure 7. Isomeric graphical structure of $\mathrm{B}_{9} \mathrm{H}_{13}$ 

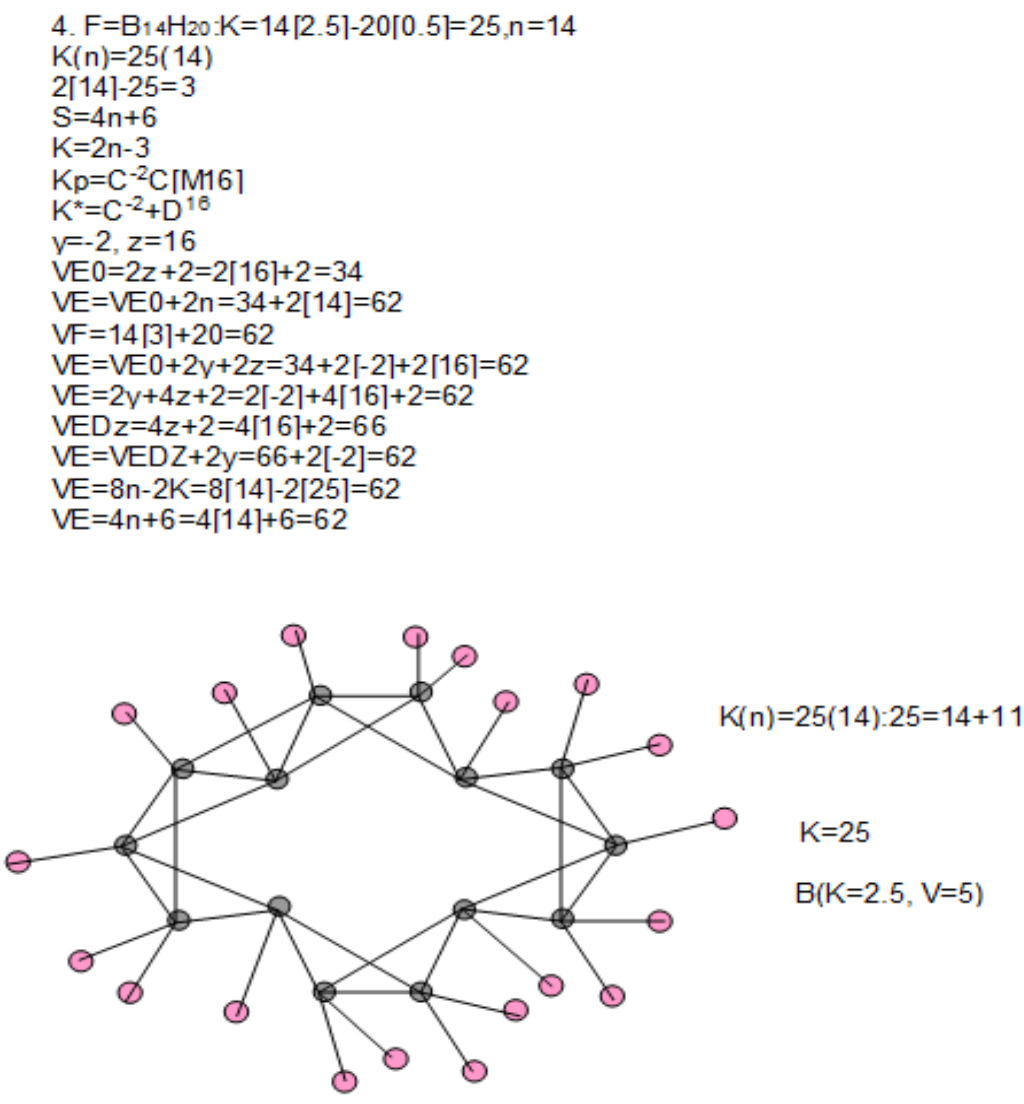

Figure 8. Isomeric graphical structure of $\mathrm{B}_{14} \mathrm{H}_{20}$

\subsection{Construction of the Skeletal Isomeric Structures}

Arising from the experience of sketching isomeric skeletal isomers of clusters, it has been found to be easier and useful to use quasi-circular skeletal structures to construct the isomers. The K(n) parameter has been very helpful. In this regard, a geometrical figure of $\mathrm{n}$ sides is selected and $\mathrm{K}$ linkages are inserted. Let us consider the following examples as illustrations. Take $\mathrm{C}_{2} \mathrm{~B}_{4} \mathrm{H}_{6}$ cluster. The $\mathrm{K}$ value for $\mathrm{C}$ is 2 and $\mathrm{V}=4$ whereas $\mathrm{B}$ has a $\mathrm{K}$ value of 2.5 and $\mathrm{V}=5$. The cluster has 11 skeletal linkages and 6 skeletal elements. In order to construct a skeletal isomer, a 6-memberered ring is selected. Then 5 linkages are added in such a way that TRIANGULAR FACES ARE FORMED. Since there are 6 skeletal elements, these can cyclically be linked by 6 linkages out of the 11 total linkages corresponding to $\mathrm{K}=11$. Hence, the remaining 5 linkages are utilized to construct the appropriate triangular faces. The triangles are constructed in such a way that the valences of the constituent skeletal elements are obeyed. This is shown in Figure 9. The same approach was done for $\mathrm{B}_{8} \mathrm{H}_{14}, \mathrm{~B}_{10} \mathrm{H}_{14}, \mathrm{C}_{2} \mathrm{~B}_{10} \mathrm{H}_{12}$ and $\mathrm{B}_{12}$ which are shown in Figures 10-17. The boron skeletal element is quite unique in that in it appears to strictly exert a skeletal valence of 5 in all its hydride clusters and other complexes such as halides, metalloboranes and metallocarboranes (Kiremire, 2017b). According to the $4 \mathrm{~N}$ series approach, we can regard the bridging hydride structures of boranes as re-arrangements of the clusters so as to achieve more stable conformations. In summary, the construction of the isomeric shapes using the $K(n)$ parameter, can be expressed as $K=n+x$ where $K=$ number of skeletal linkages, $\mathrm{n}=$ the number of the skeletal elements and $\mathrm{x}=$ the number of linkages inserted within and/or around the $\mathrm{n}$-sided figure in such a way that skeletal TRIANGLES are generated. The parent structures on which linkage lines are drawn are based on Symyx Draw 3.2 program. 


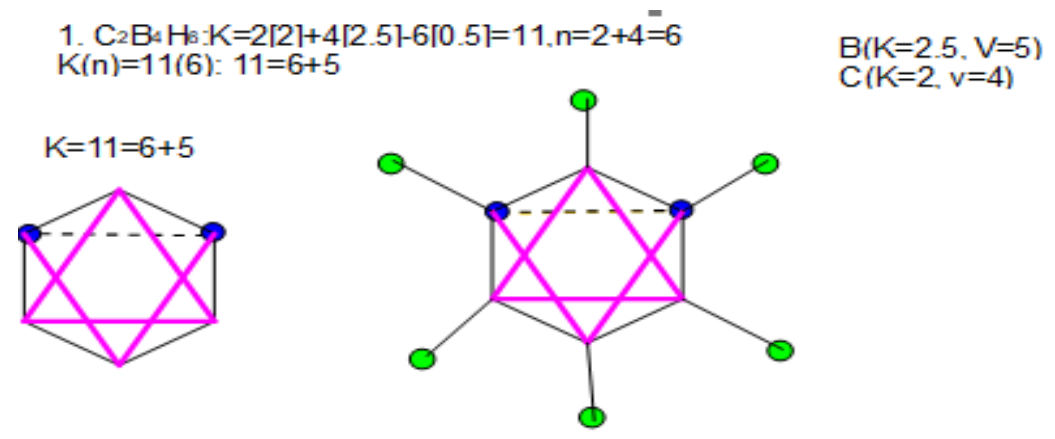
- - - = inserted for completenessto be in aqreement with the conventional On qeometrv not constructed accordinq to the $4 \mathrm{~N}$ series method.

Figure 9. Isomeric graphical structure of $\mathrm{C}_{2} \mathrm{~B}_{4} \mathrm{H}_{6}$

2. $\mathrm{B}_{8} \mathrm{H}_{14}: \mathrm{K}=8[2.5]-14[0.5]=13, \mathrm{n}=8$

$K(n)=13(8) ; 13=8+5$

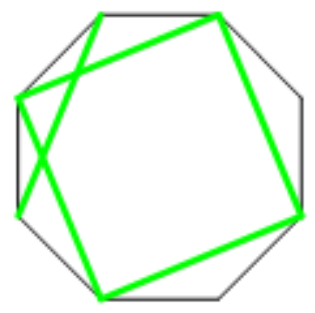

$13=8+5$

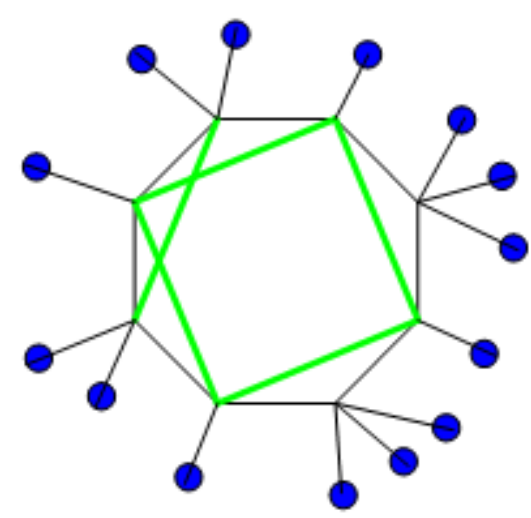

Figure 10. Isomeric graphical structure of $\mathrm{B}_{8} \mathrm{H}_{14}$

3. $\mathrm{B}_{10} \mathrm{H}_{14}: \mathrm{K}=10[2.5]-14[0.5]=18, \mathrm{n}=10$

$K(n)=18(10) ; 18=10+8$

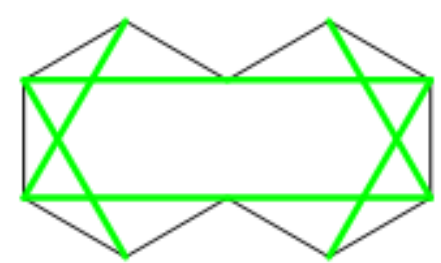

$18=10+8$

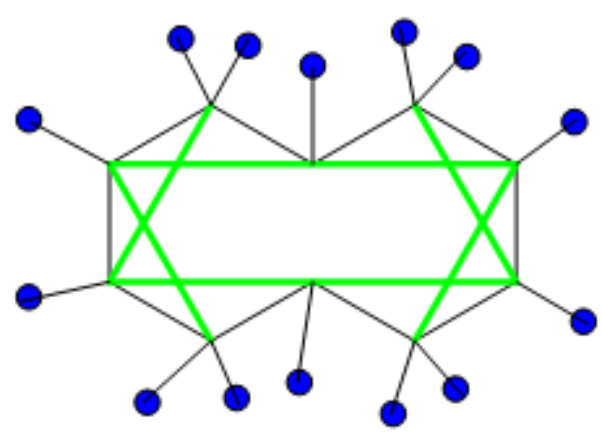

Figure 11. Isomeric graphical structure of $\mathrm{B}_{10} \mathrm{H}_{14}$ 
4. $\mathrm{C}_{2} \mathrm{~B}_{10} \mathrm{H}_{12} \cdot \mathrm{K}=2[2]+10[2.5]-12[0.5]=23, \mathrm{n}=2+10=12$

$\mathrm{K}(\mathrm{n})=23(12): 23=12+11$
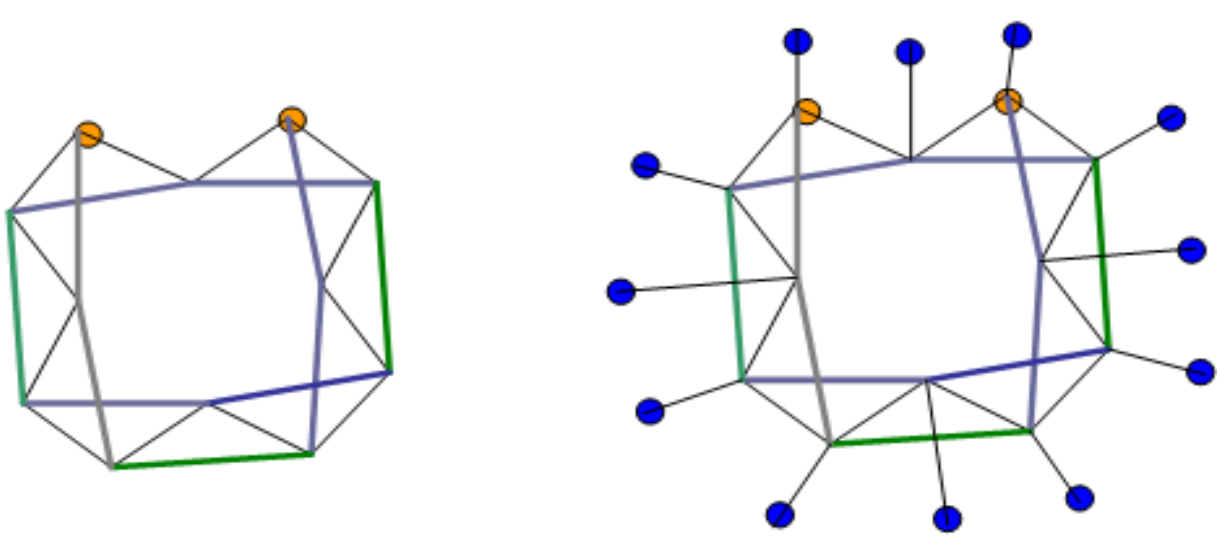

Figure 12. Isomeric graphical structure of $\mathrm{C}_{2} \mathrm{~B}_{10} \mathrm{H}_{12}$

5. $B_{12}=12[2.5]=30, n=12$

$K(n)=30(12) ; 30=12+18$

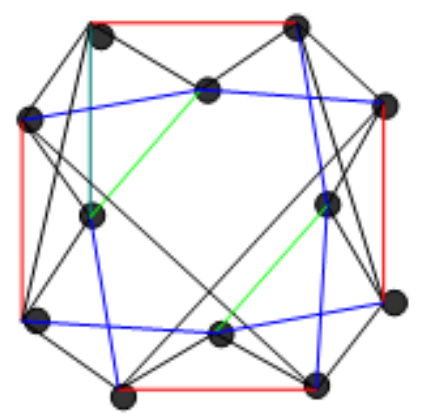

Figure 13. Isomeric graphical structure of $\mathrm{B}_{12}$

6. $\mathrm{B}_{8} \mathrm{Cl}_{8}: \mathrm{K}=8[2.5]-8[0.5]=16, \mathrm{n}=8$

$\mathrm{K}(\mathrm{n})=16(8) ; 16=8+8$

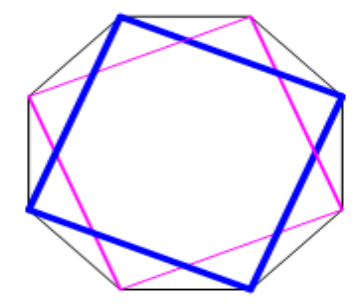

$8+8$

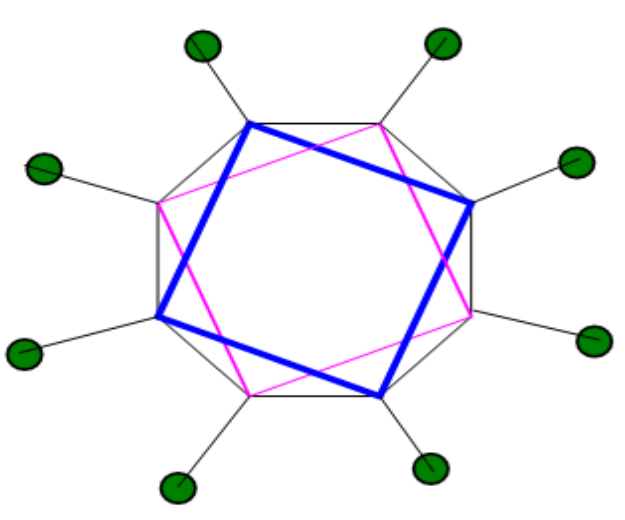

Figure 14. Isomeric graphical structure of $\mathrm{B}_{12} \mathrm{Cl}_{8}$ 
7. $\mathrm{B}_{9} \mathrm{Cl} \cdot \mathrm{K}=9[2.5]-9[0.5]=18, \mathrm{n}=9$ $K(n)=18(9) ; 18=9+9$

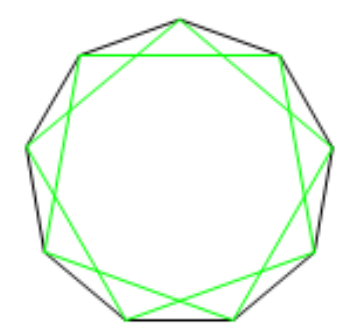

$9+9$

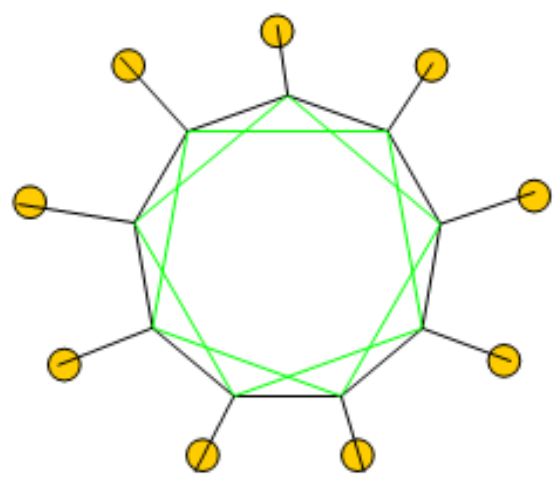

Figure 15. Isomeric graphical structure of $\mathrm{B}_{9} \mathrm{Cl}_{9}$

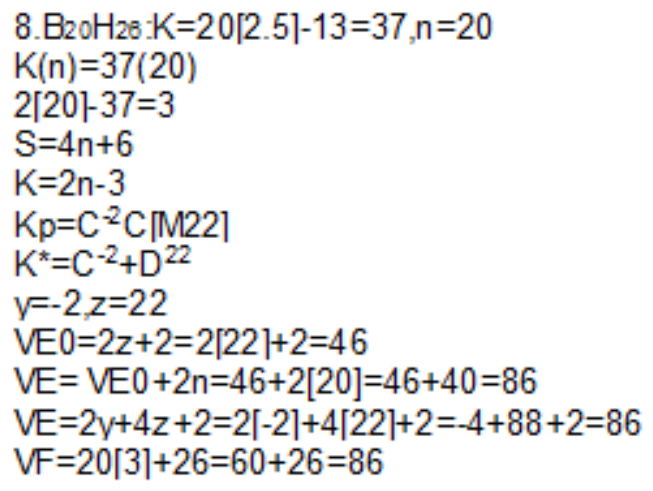

$\mathrm{K}=37$

$37=20+17$

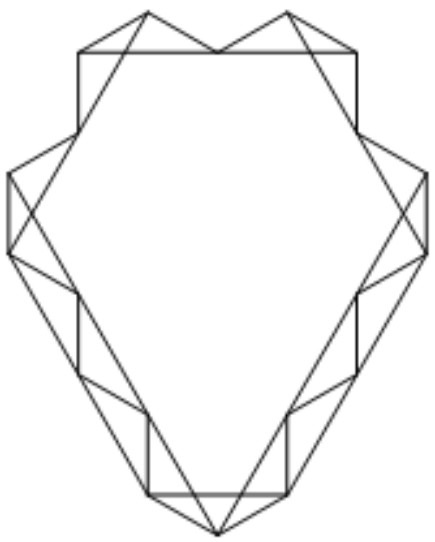

$\mathrm{B}(\mathrm{K}=2.5, \mathrm{~V}=5)$

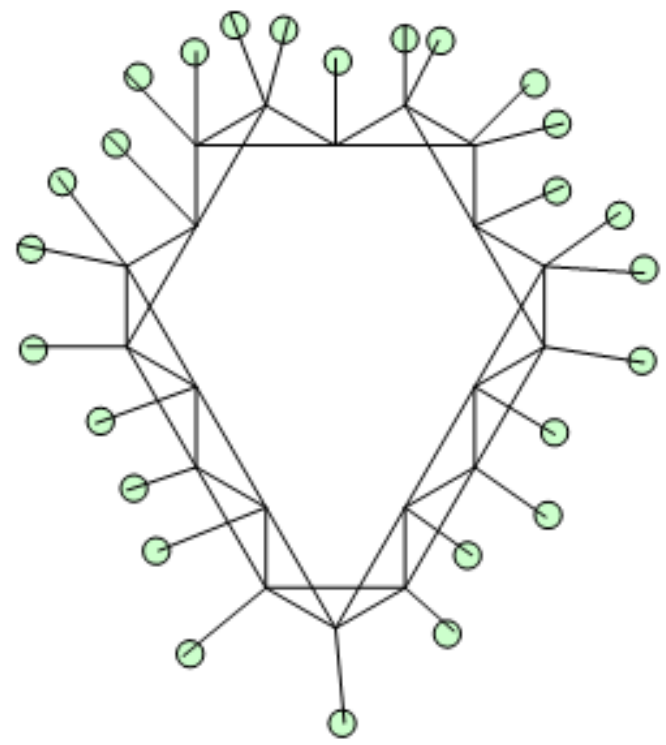

Figure 16. Isomeric graphical structure of $\mathrm{B}_{20} \mathrm{H}_{26}$ 


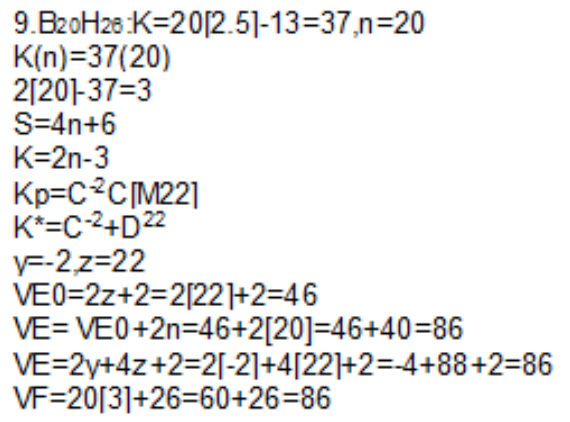

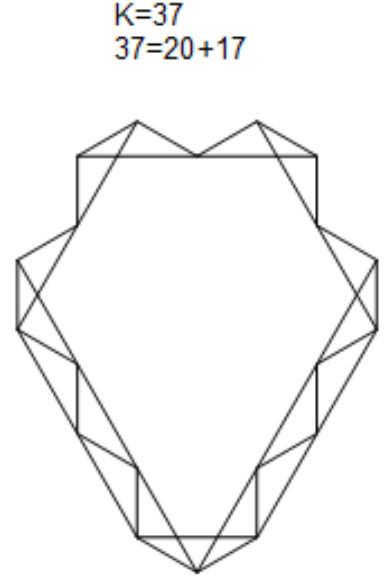

$\mathrm{B}(\mathrm{K}=2.5, \mathrm{~V}=5)$

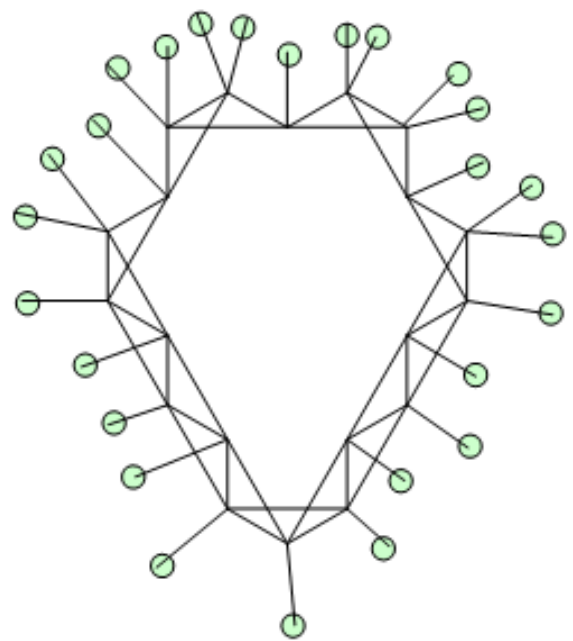

Figure 17. Isomeric graphical structure of $\mathrm{B}_{20} \mathrm{H}_{26}$

\section{Categorization of Borane Clusters}

A systematic method of categorization of clusters was recently developed (Kiremire 2019a-c). According to the method, a categorization parameter $\mathrm{K}^{*}=\mathrm{C}^{\mathrm{y}}+\mathrm{D}^{\mathrm{z}}$ was introduced where $\mathrm{y}+\mathrm{z}=\mathrm{n}=$ normally is number of skeletal elements in the cluster and $\mathrm{C}=$ represents the outer capping symbol and $\mathrm{D}$ represents the inner capping symbol of the cluster as it was discovered that clusters and chemical elements portray double capping phenomena. The symbol $\mathrm{D}^{\mathrm{z}}$ is also regarded as representing CLAN series of the clusters (Kiremire, 2019a-c). The analyzed boranes fall within the range $\mathrm{D}^{1}-\mathrm{D}^{22}$ cluster clans and are shown in Table 1. The capping is in line with the descending cluster series that Rudolph identified more than 40 years ago (Rudolph, 1976).

\subsection{The Evolution of Isomeric Polyhedral Geometries}

During the analysis of borane clusters including the construction of clusters shapes with nuclearity index of 5 and above, it has been found helpful to utilize ring structures corresponding to the level of the nuclearity index. This means that for example $\mathrm{B}_{5}$ clusters, a 5-membered ring is used, $\mathrm{B}_{6}$ clusters, we use a 6-membered ring, $\mathrm{B}_{7}, 7$-membered ring, $\mathrm{B}_{8}, 8$ membered ring and so on. According to this approach, there is a relationship between the cluster skeletal linkages $\mathrm{K}$ and the number of skeletal elements, $n$. This relationship is given between by $\mathrm{K}=\mathrm{n}+\mathrm{x}$ where $\mathrm{x}=$ number of skeletal lines or triangles constructed. As this relationship is considered very important, is being repeated here for emphasis. Thus, for $K(n)=7(6), 7=6+1$ and therefore a 6 -membered ring with one triangle constructed inside will fulfill the cluster valence content of all the hydrogen ligands in the cluster. This is the case for $\mathrm{B}_{6} \mathrm{H}_{16}$ shown in Figure 18-0. The cluster $\mathrm{B}_{6} \mathrm{H}_{14}$ with $\mathrm{K}(\mathrm{n})=8(6) ; 8=6+2$, we will construct two triangles to be in resonance with the formula $\mathrm{B}_{6} \mathrm{H}_{14}$. This is shown in Figure 19 . The procedure goes on for $\mathrm{B}_{6} \mathrm{H}_{12}, \mathrm{~K}(\mathrm{n})=9(6) ; 9=6+3 \rightarrow 3$ triangles; $\mathrm{B}_{6} \mathrm{H}_{10}, \mathrm{~K}(\mathrm{n})=10(6) ; 10=6+4 \rightarrow 4$ triangles, $\mathrm{B}_{6} \mathrm{H}_{8}\left[\mathrm{~B}_{6} \mathrm{H}_{6}{ }^{2-}\right] \rightarrow \mathrm{K}(\mathrm{n})=11(6) ; 11=6+5 \rightarrow 5$ triangles, $\mathrm{B}_{6} \mathrm{H}_{6}, \mathrm{~K}(\mathrm{n})=12(6)$; and $12=6+6 \rightarrow 6$ triangles. The increasing number of skeletal triangles are given in Figures 20-27. The $\mathrm{K}(\mathrm{n})=12(6)$ value for $\mathrm{B}_{6} \mathrm{H}_{6}$ is the same as that of $\mathrm{C}_{6}$ and $\mathrm{Os}_{6}(\mathrm{CO})_{18}$. Therefore, these clusters have the same skeletal structure. These are shown in Scheme 1. 
1.

$\mathrm{B}_{6} \mathrm{H}_{18}: \mathrm{K}=6[2.5]-18[0.5]=15-9=6, \mathrm{n}=6$

$K(n)=6(6) ; 6=6+0$

$2[n]-K=2[6]-6=6,-S=4 n+12, K=2 n-6, K p=C^{-5} C[M 11]$

$\mathrm{K}^{*}=\mathrm{C}^{-5}+\mathrm{D}^{11}$
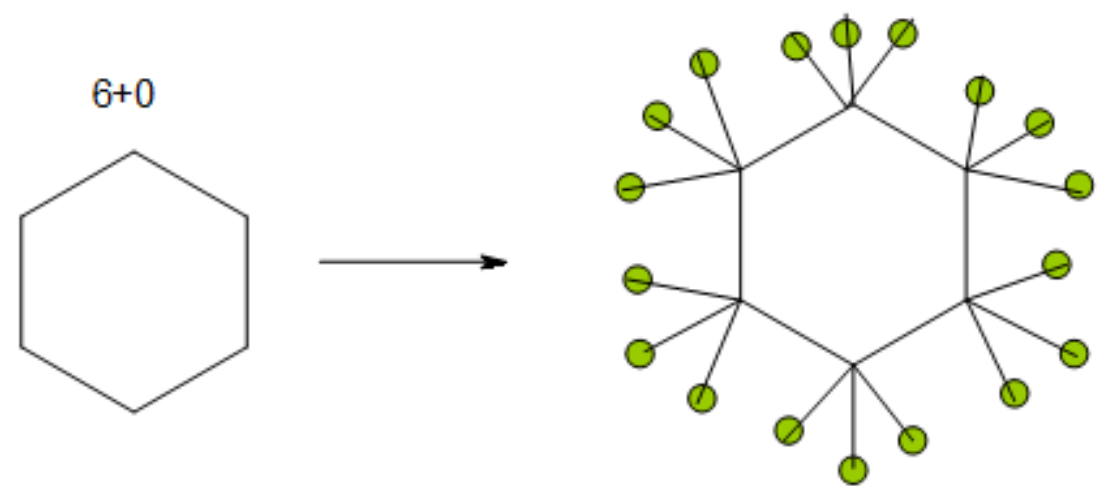

Figure 18. Isomeric graphical structure of $\mathrm{B}_{6} \mathrm{H}_{16}$

2. $\mathrm{F}=\mathrm{B}_{6} \mathrm{H}_{16}: \mathrm{K}=6[2.5]-8=7, \mathrm{n}=6$

$$
\begin{aligned}
& \mathrm{K}(\mathrm{n})=7(6) \\
& 2[6]-7=5 \\
& \mathrm{~S}=4 \mathrm{n}+10 \\
& \mathrm{~K}=2 \mathrm{n}-5 \\
& \mathrm{Kp}=\mathrm{C}^{-4} \mathrm{C}[\mathrm{M} 10] \\
& \mathrm{K}^{*}=\mathrm{C}^{-4}+\mathrm{D}^{10} \\
& \mathrm{y}=-4, \mathrm{z}=10 \\
& \mathrm{VE} 0=2 \mathrm{z}+2=2[10]+2=22 \\
& \mathrm{VEDz}=4 \mathrm{z}+2=4[10]+2=42 \\
& \mathrm{VE}=\mathrm{VE} 0+2 \mathrm{n}=22+2[6]=22+12=34 \\
& \mathrm{VF}=6[3]+16=18+16=34 \\
& \mathrm{VE}=\mathrm{VE} 0+2 \mathrm{y}+2 \mathrm{z}=22+2[-4]+2[10]=34 \\
& \mathrm{VE}=\mathrm{VED} \mathrm{z}+2 \mathrm{y}=42+2[-4]=34 \\
& \mathrm{VE}=2 \mathrm{y}+4 \mathrm{z}+2=2[-4]+4[10]+2=34 \\
& \mathrm{VE}=8 \mathrm{n}-2 \mathrm{~K}=8[6]-2[7]=34 \\
& \mathrm{VE}=4 \mathrm{n}+10=4[6]+10=34
\end{aligned}
$$




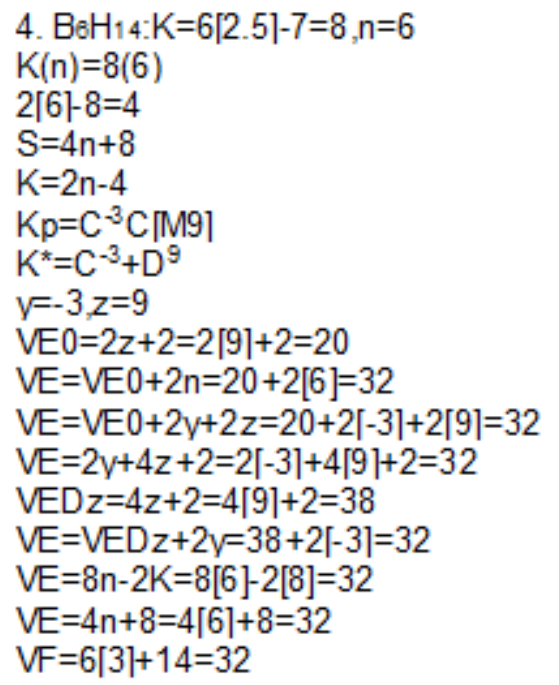

\section{$\mathrm{B}_{6} \mathrm{H}_{14}$}

$\mathrm{K}=8$

$\mathrm{B}(\mathrm{K}=2.5, \mathrm{~V}=5)$

$K(n)=8(6) \quad 8=6+2$
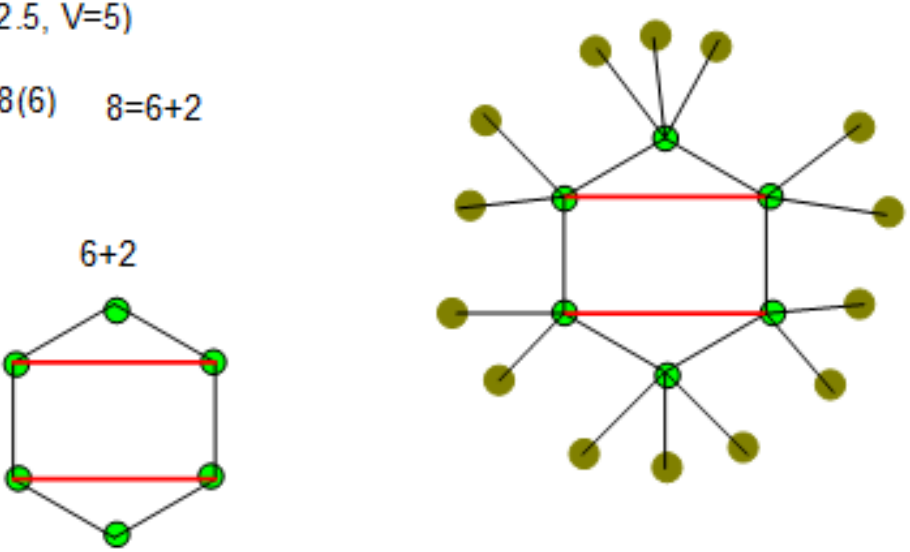

Figure 19. Isomeric graphical structure of $\mathrm{B}_{6} \mathrm{H}_{14}$

5. $\mathrm{B}_{6} \mathrm{H}_{12}: \mathrm{K}=6[2.5+-6=9, \mathrm{n}=6$ $\mathrm{K}(\mathrm{n})=9(6)$ $9=6+3$
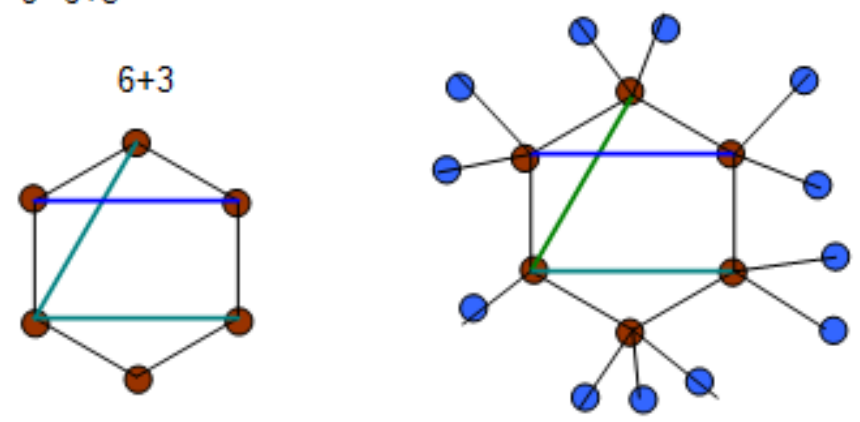

$\mathrm{K}=9$

$B(K=2.5, V=5)$

$\mathrm{B}_{6} \mathrm{H}_{12}$

Figure 20. Isomeric graphical structure of $\mathrm{B}_{6} \mathrm{H}_{12}$ 


\section{6. $\mathrm{B}_{6} \mathrm{H}_{10} \mathrm{~K}=6[2.5+-5=10, \mathrm{n}=6$ $\mathrm{K}(\mathrm{n})=10(6)$ \\ $10=6+4$}
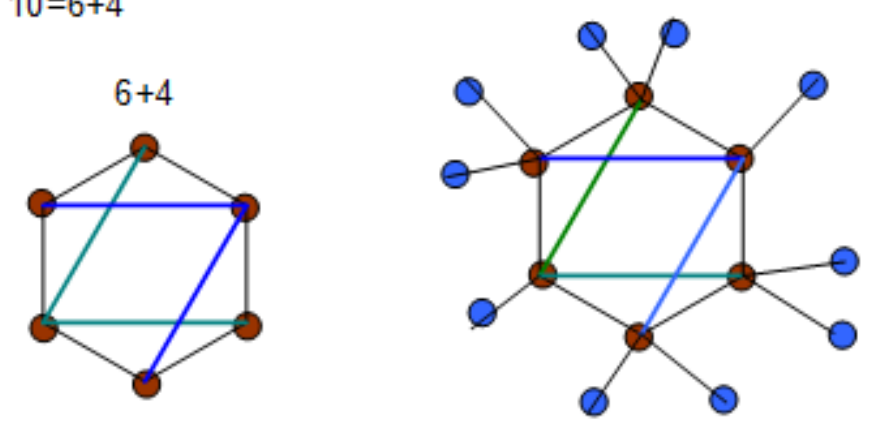

$$
\begin{aligned}
& K=10 \\
& 10=6+4
\end{aligned}
$$

\section{$\mathrm{B}_{6} \mathrm{H}_{10}$}

Figure 21. Isomeric graphical structure of $\mathrm{B}_{6} \mathrm{H}_{10}$

7. $\mathrm{B}_{6} \mathrm{H}_{8}-\mathrm{K}=6[2.5 \mathrm{l}-4=11, \mathrm{n}=6$

$\mathrm{K}(\mathrm{n})=11(6)$

$11=6+5$

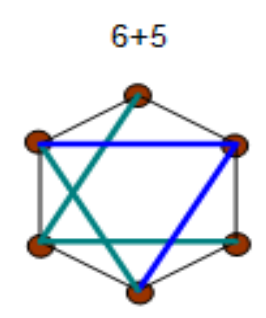

$\mathrm{K}=11$

$11=6+5$

$\mathrm{B}(\mathrm{K}=2.5, \mathrm{~V}=5)$

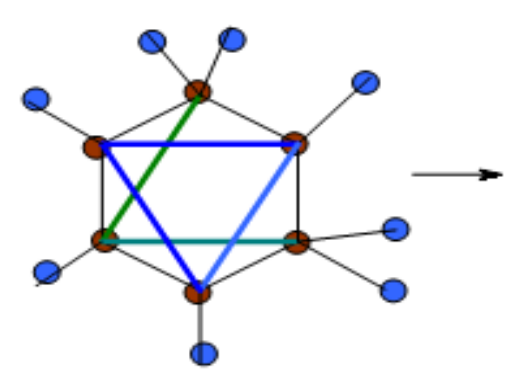

$\mathrm{B} 8 \mathrm{H}_{8}$

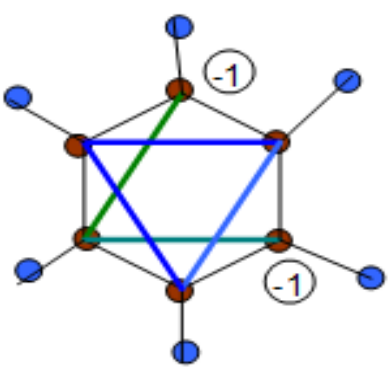

$\mathrm{B}_{6} \mathrm{H}_{6}{ }^{2-}$

Figure 22. Isomeric graphical structure of $\mathrm{B}_{6} \mathrm{H}_{8}$

(-1)

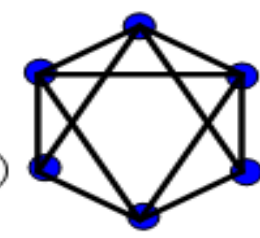

(-1)

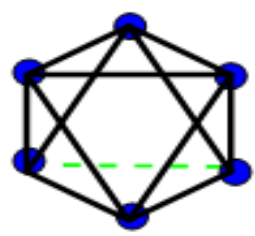

Linking up the nodal charges to get a closed shape

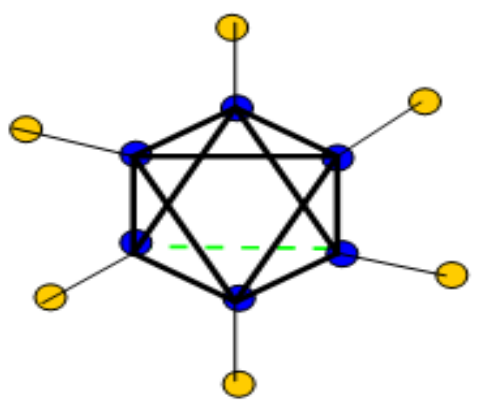

Figure 23. Isomeric graphical structure of $\mathrm{B}_{6} \mathrm{H}_{6}{ }^{2-}$ 
As H Ligands Decrease, K Increases and More Triangles Are Formed.

3.2 Closo Capping Series
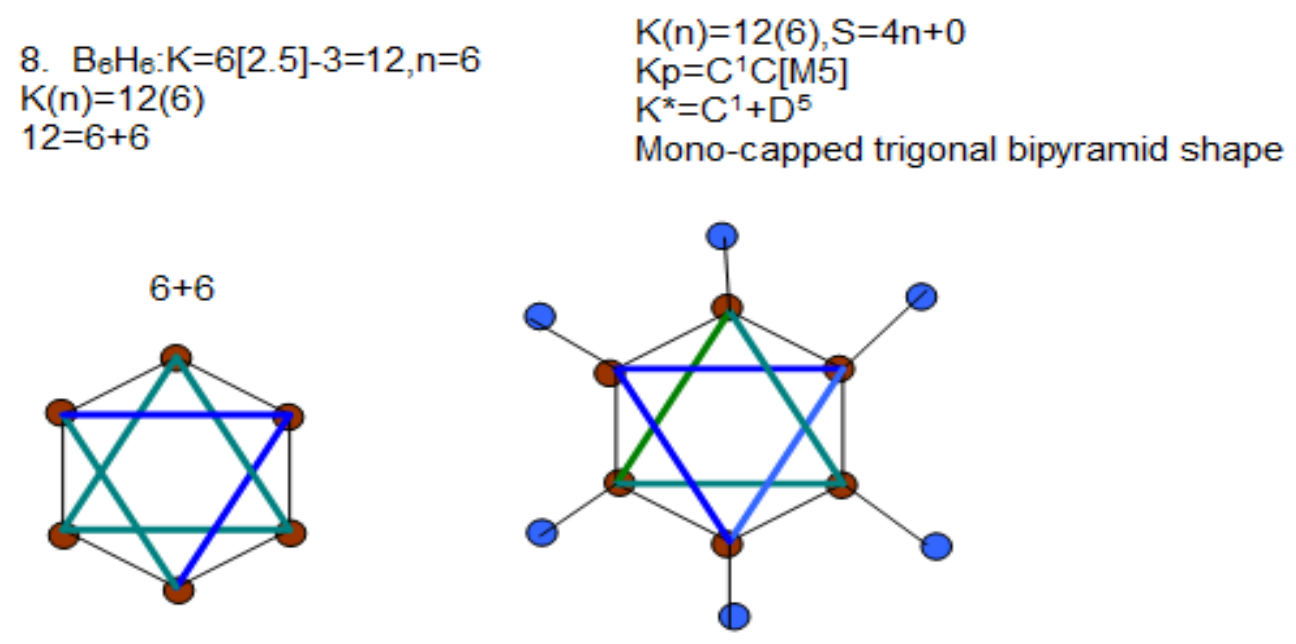

$$
\begin{aligned}
& \mathrm{K}=12 \\
& 12=6+6 \\
& \mathrm{~B}(\mathrm{~K}=2.5, \mathrm{~V}=5)
\end{aligned}
$$

$$
\mathrm{B}_{6} \mathrm{H}_{6}
$$

Figure 24. Isomeric graphical structure of $\mathrm{B}_{6} \mathrm{H}_{6}$

$$
\begin{aligned}
& \mathrm{B}_{6} \mathrm{H}_{4}: \mathrm{K}=6[2.5]-2=13, \mathrm{n}=6 \\
& \mathrm{~K}(\mathrm{n})=13(6) ; 13=6+7
\end{aligned}
$$

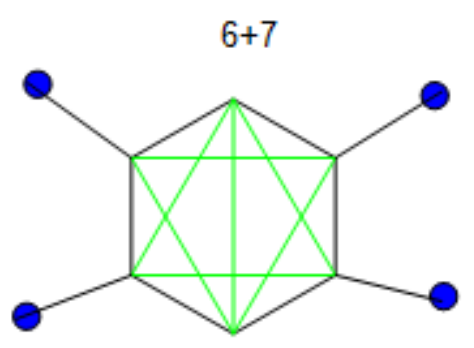

$K(n)=13(6)$

$2[6]-13=-1$

$\mathrm{S}=4 \mathrm{n}-2$

$K=2 n+1$

$\mathrm{Kp}=\mathrm{C}^{2} \mathrm{C}[\mathrm{M} 4]$

$K^{*}=C^{2}+D^{4}$

Figure 25. Isomeric graphical structure of $\mathrm{B}_{6} \mathrm{H}_{4}$

$\mathrm{B}_{6} \mathrm{H}_{2} \cdot \mathrm{K}=6[2.5 \mathrm{r}-1=14, \mathrm{n}=6$
$\mathrm{K}(\mathrm{n})=14(6) ; 13=6+8$

$$
\begin{aligned}
& K(n)=14(6) \\
& 2[6+-14=-2 \\
& S=4 n-4 \\
& K=2 n+2 \\
& K p=C^{3} C[M 3] \\
& K^{\star}=C^{3}+D^{3} \\
& \text { Tri-capped triangle shape }
\end{aligned}
$$

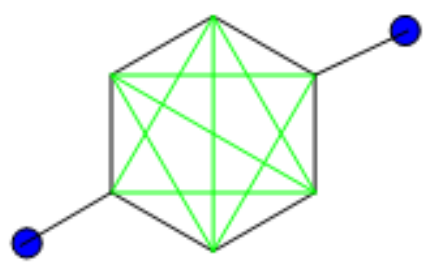

Figure 26. Isomeric graphical structure of $\mathrm{B}_{6} \mathrm{H}_{2}$ 


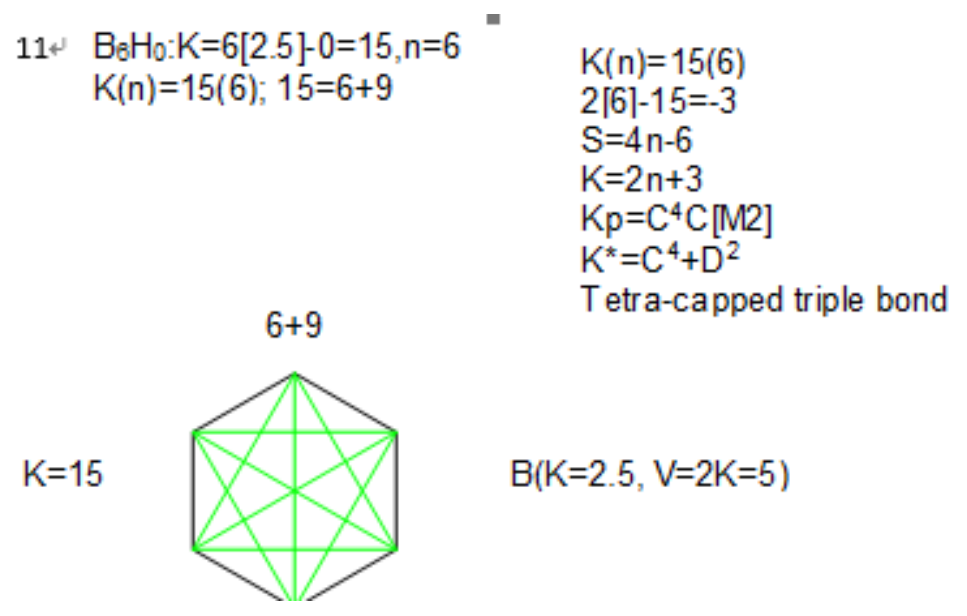

Figure 27. Isomeric graphical structure of $\mathrm{B}_{6} \mathrm{H}_{0}=\mathrm{B}_{6}$

3.3 Equivalent Isomeric Skeletal Mono-Capping Clusters

$\mathrm{B}_{6} \mathrm{H}_{6}$

$K=6[2.5]-6[0.5=12, n=6$

$\mathrm{K}(\mathrm{n})=12(6)$

$2[6]-12=0$

$\mathrm{S}=4 \mathrm{n}+0$

$\mathrm{K}=2 \mathrm{n}+0$

$\mathrm{Kp}=\mathrm{C}^{1} \mathrm{C}[\mathrm{M} 5]$

$\mathrm{K}^{*}=\mathrm{C}^{1}+\mathrm{D}^{5}$

$y=1, z=5$

VE $0=2 z+2=2[5]+2=12$

$\mathrm{VE}=\mathrm{VE} 0+2 \mathrm{n}=12+2[6]=12+12=24$

$\mathrm{VF}=6[3]+6=24$

$V E=2 y+4 z+2=2[1]+4[5]+2=24$

$\mathrm{Os}_{6}(\mathrm{CO})_{18}$

$K=6[5]-18[1]=12, n=6$

$K(n)=12(6)$

Same as $\mathrm{B}_{6} \mathrm{H}_{6}$

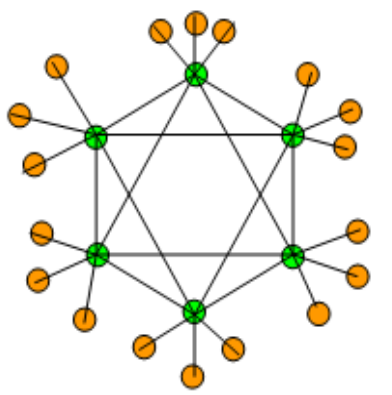

$\mathrm{Os}_{6}(\mathrm{CO})_{18}$

$\mathrm{Os}(\mathrm{K}=5, \mathrm{~V}=10)$
$\mathrm{C}_{6}$

$\mathrm{K}=6[2]=12, \mathrm{n}=6$

$K(n)=12(6)$

The same as $\mathrm{B}_{6} \mathrm{H}_{6}$.

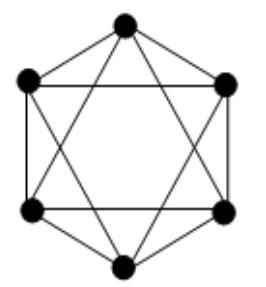

$\mathrm{C}_{6}$

$\mathrm{C}(\mathrm{K}=2, \mathrm{~V}=4)$

$K(n)=12(6): 12=6+6$

Scheme 1. Equivalent Isomeric graphical mono-capped closo structures of $\mathrm{B}_{6} \mathrm{H}_{6}, \mathrm{C}_{6}$ and $\mathrm{Os}_{6}(\mathrm{CO})_{18}$

Since $\mathrm{B}_{6} \mathrm{H}_{6}, \mathrm{C}_{6}$ and $\mathrm{Os}_{6}(\mathrm{CO})_{18}$ have the same $\mathrm{K}(\mathrm{n})$ value, we can regard them as being equivalent SKELETAL ISOMERS. Since the CO ligand is a two-electron donor, it can be considered as using up 2 skeletal linkages of osmium skeletal element. The carbon skeletal element has 4 skeletal linkages which are all utilized in forming the isomeric skeletal structure of $\mathrm{C}_{6}$.

\subsection{The Closo Series: $B_{n} H_{n}^{2-}$}

The construction of the closo structures is similar to the general one of constructing other non-closo structures. The guidance is simply the $\mathrm{K}(\mathrm{n})$ parameter of the cluster. First, an n-sided ring is selected. Then additional linkages are added 
to the ring in such a way that the total linkages are the same as the numerical number of skeletal linkages $\mathrm{K}$. That is, $\mathrm{K}=\mathrm{n}+\mathrm{x}$ where $\mathrm{x}$ represents the number of additional linkages constructed in such a way that triangles are created including the nodal points carrying the negative charges. This procedure is shown in Scheme 2 for the closo $\mathrm{B}_{5} \mathrm{H}_{5}^{2-}$. In closo structures, the points with negative charges are linked by a line sealing off a triangle. The closo structures of selected boranes B5-15, B19-20 are given Figures 28 and 29.

5-membered ring

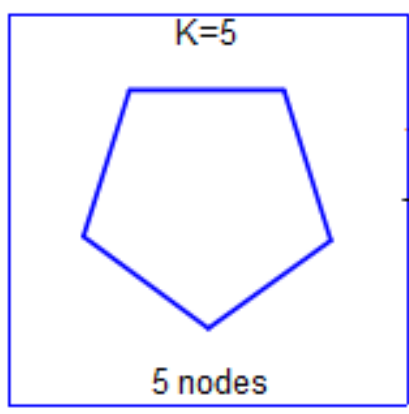

$K=5+4=9$

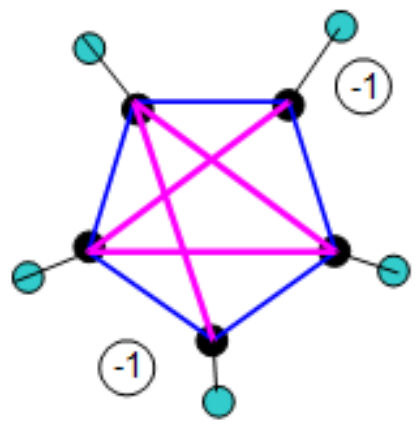

$+5 \mathrm{H}$ ligands

$\mathrm{B}_{5} \mathrm{H}_{5}{ }^{2-}$
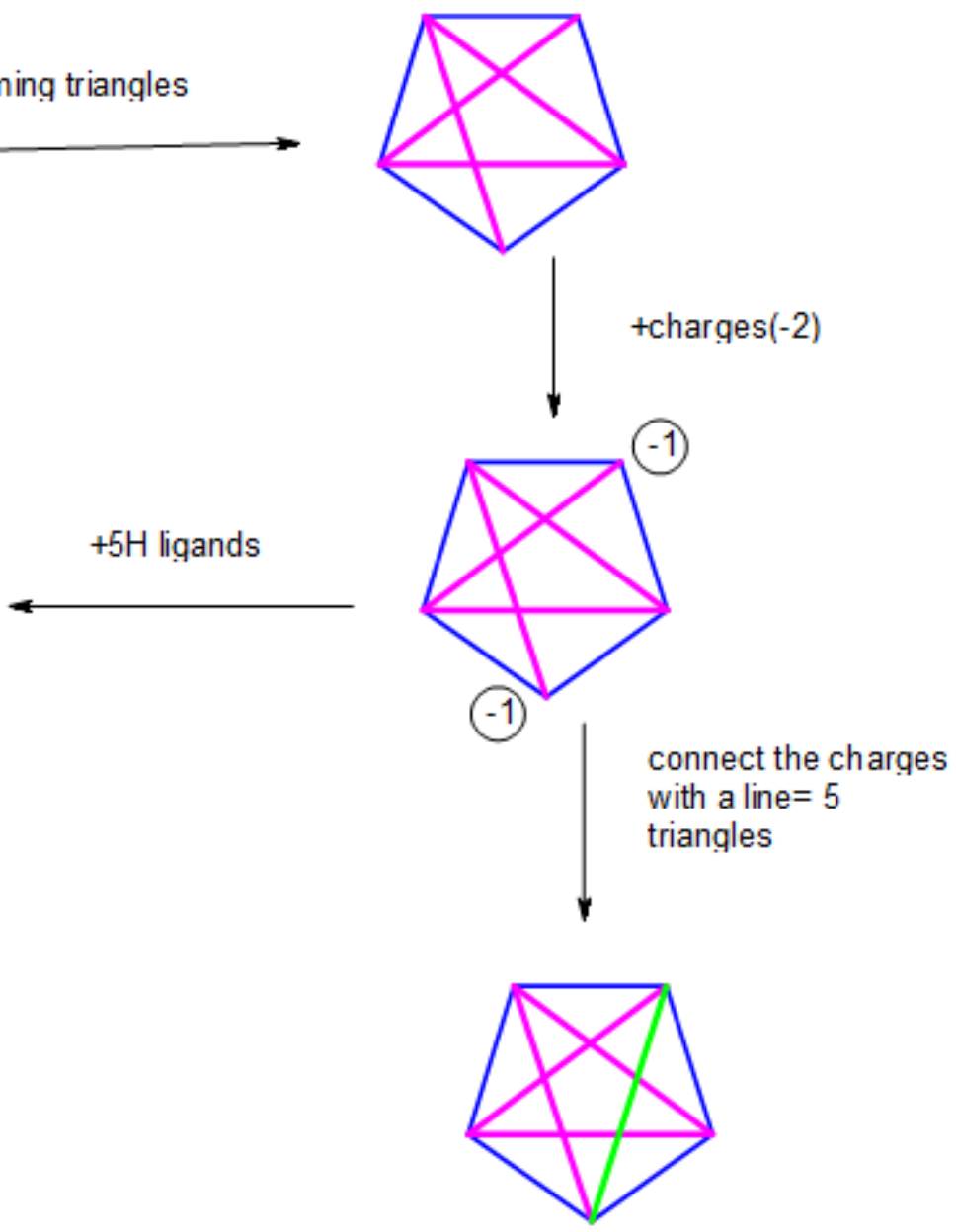

CLOSED STRUCTURE $=$ CLOSO STRUCTURE OF $\mathrm{B}_{5} \mathrm{H}_{5}{ }^{2-}$

Scheme 2. Construction of the formation of a closo structure of $\mathrm{B}_{5} \mathrm{H}_{5}{ }^{2-}$ 


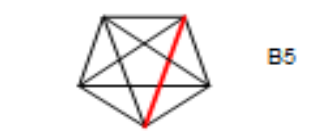

$K=2 n-1=2|5|-1=9 ;-K\langle n|=9| 5|, K=n+x=5+4$.
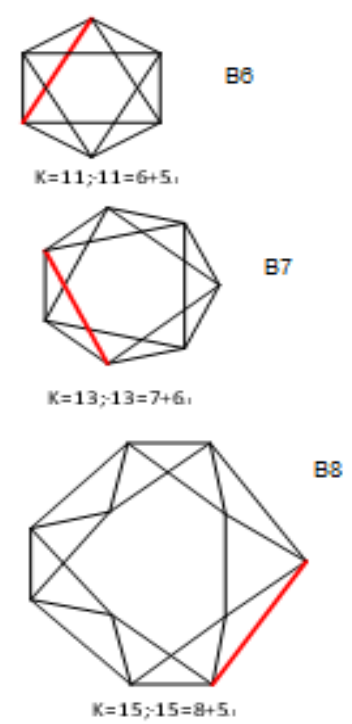

B8
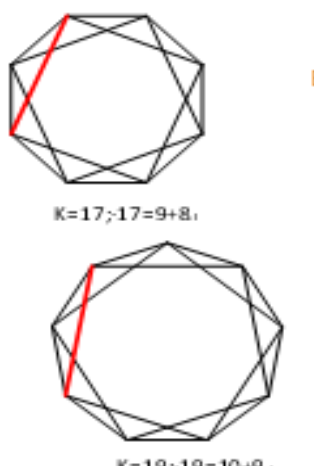

$K=19 ;-19=10+9$.
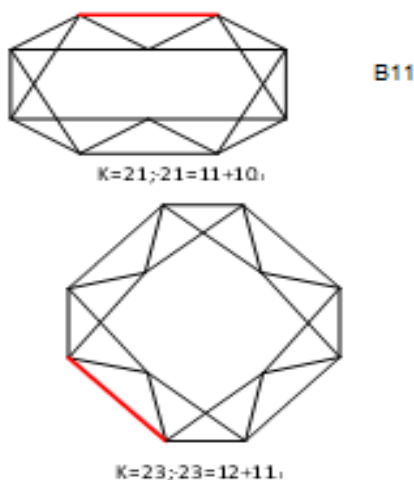

$\mathrm{B9}$

B10

11

B12

Figure 28. Closo isomeric structures of $\mathrm{B}_{5}$ to $\mathrm{B}_{12}$
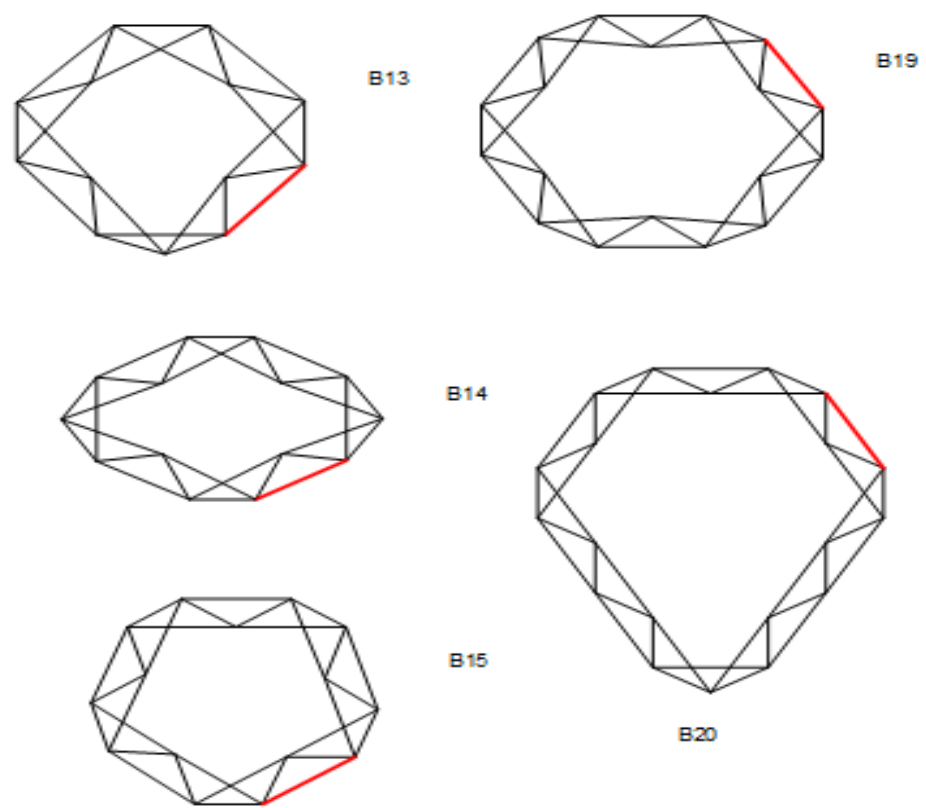

Figure 29. Isomeric graphical structure of $B_{13}$ to $B_{15}, B_{19}$ - $B_{20}$

\section{Descending Borane Clan Series $\left(\mathrm{D}^{\mathrm{z}}\right)$}

\subsection{General Descending Series}

A wide range of clusters have been categorized into clan series using skeletal numbers. More than 60 borane clusters have been ranging from $\mathrm{D}^{5}$ to $\mathrm{D}^{22}$. Their analysis is given examples 1-51 some of their isomeric graphical shapes in Figures154 and are presented in Table 1. 


\subsection{The popular Rudolph Clan Series}

Clusters have broadly been categorized (Kiremire,2018). Those which change by $\Delta K= \pm 3$ and $\Delta \mathrm{n}= \pm 1$ are in line with the cluster relationship which was brilliantly identified by Rudolph more than 40 years ago (Rudolph,1976).

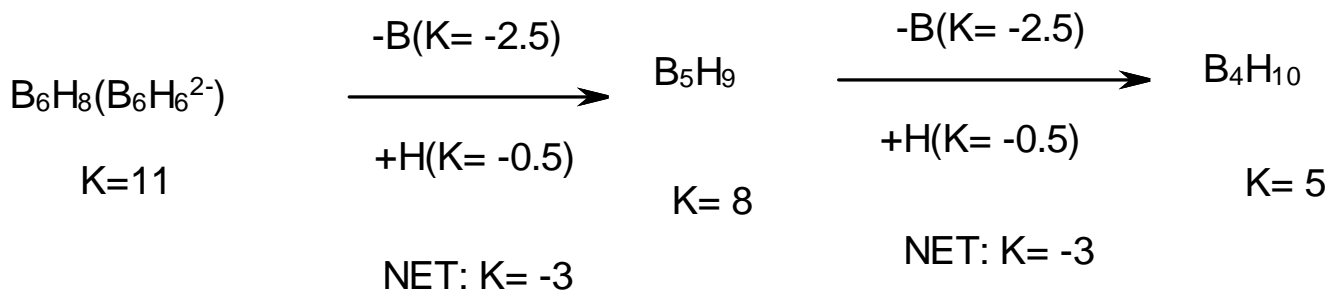

The Rudolph clan series behave as if a boron (B) skeletal element is transmuted into a hydrogen(H) ligand at each descending step. Also, a closo skeletal structure is obtained when the NODAL points bearing negative charges are linked up to generate an additional triangular face. The changes in the skeletal structure are shown in Scheme 3.

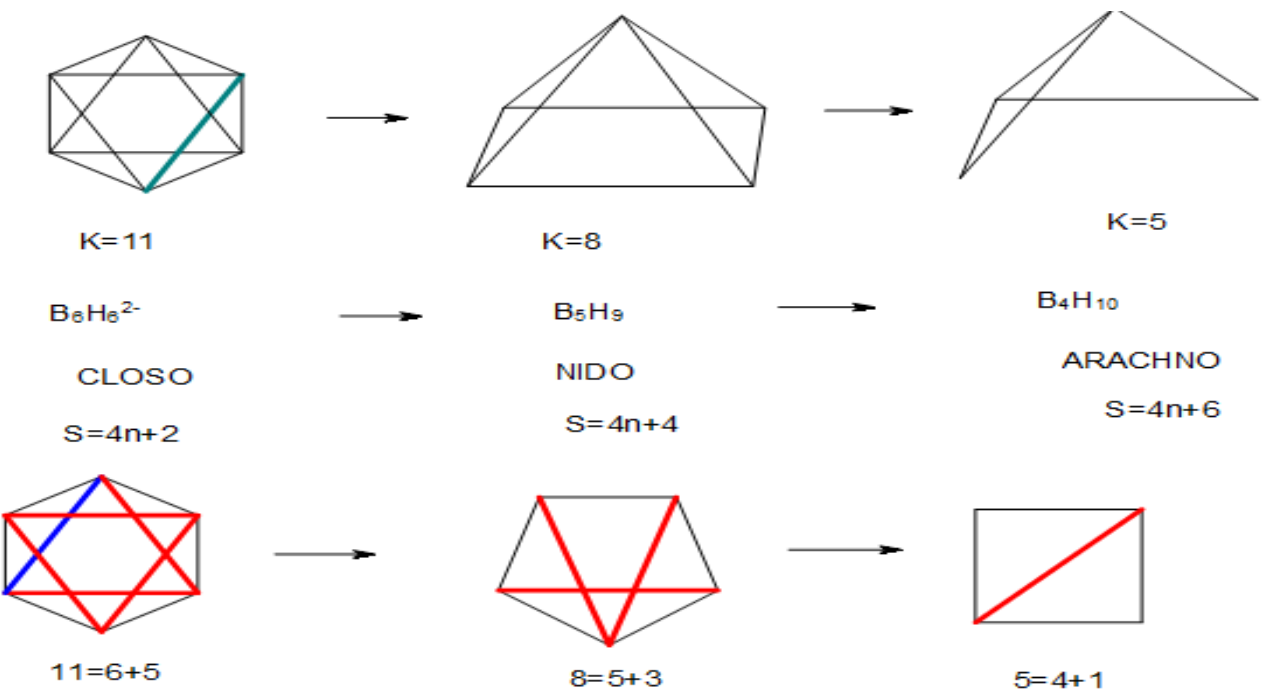

Scheme 3. Isomeric graphical structures of $\mathrm{B}_{6} \mathrm{H}_{6}{ }^{2-}, \mathrm{B}_{5} \mathrm{H}_{9}^{-}, \mathrm{B}_{4} \mathrm{H}_{10}$

$\mathrm{D}^{5}$ SERIES

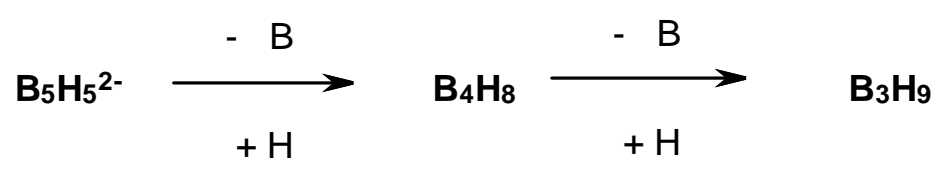

11. $\mathrm{F}=\mathrm{B}_{5} \mathrm{H}_{5}^{2-:} \mathrm{K}=5[2.5]-5[0.5]-2[0.5]=9, \mathrm{n}=5$

$\mathrm{K}(\mathrm{n})=9(5)$

$10-9=1$

$\mathrm{S}=4 \mathrm{n}+2$ (closo)

$\mathrm{K}=2 \mathrm{n}-1$

$\mathrm{Kp}=\mathrm{C}^{0} \mathrm{C}[\mathrm{M} 5]$

$\mathrm{K}^{*}=\mathrm{C}^{0}+\mathrm{D}^{5}$

$\mathrm{y}=0, \mathrm{z}=5$

$\mathrm{VE} 0=2 \mathrm{z}+2=2[5]+2=12$

$\mathrm{VE}=\mathrm{VE} 0+2 \mathrm{n}=12+2[5]=22$

$\mathrm{VE}=4 \mathrm{n}+2=4[5]+2=22$

$\mathrm{VF}=5[3]+5+2=22$ 
$\mathrm{K}=9$

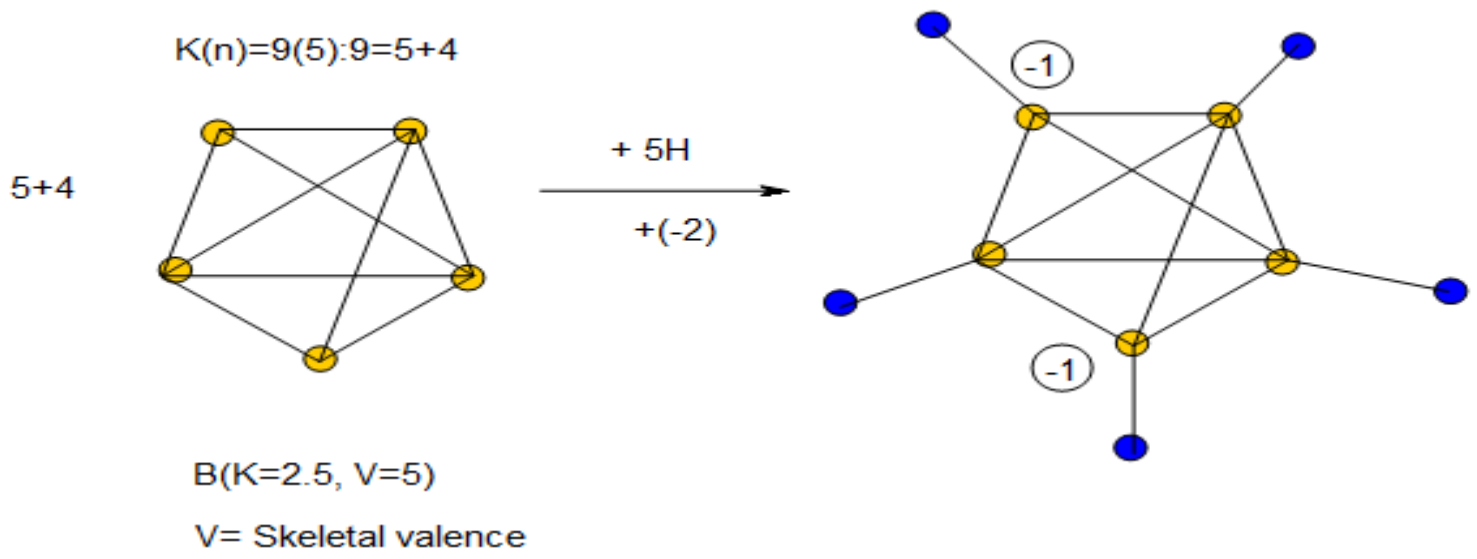

Figure 30. Isomeric graphical structure of $\mathrm{B}_{5} \mathrm{H}_{5}^{2-}$

12. $\mathrm{F}=\mathrm{B}_{4} \mathrm{H}_{8}: \mathrm{K}=4[2.5]-8[0.5]=6, \mathrm{n}=4$

$\mathrm{K}(\mathrm{n})=6(4)$

$8-6=2$

$\mathrm{S}=4 \mathrm{n}+4$ (nido)

$\mathrm{K}=2 \mathrm{n}-2$

$\mathrm{Kp}=\mathrm{C}^{-1} \mathrm{C}[\mathrm{M} 5]$

$\mathrm{K}^{*}=\mathrm{C}^{-1}+\mathrm{D}^{5}$

$\mathrm{y}=-1, \mathrm{z}=5$

$\mathrm{VE} 0=2 \mathrm{z}+2=2[5]+2=12$

$\mathrm{VE}=\mathrm{VE} 0+2 \mathrm{n}=12+2[4]=12+8=20$

$\mathrm{VF}=4[3]+8=20$

$\mathrm{VE}=4 \mathrm{n}+4=4[4]+4=20$

$$
\mathrm{K}=6
$$

$K(n)=6(4): 6=4+2$
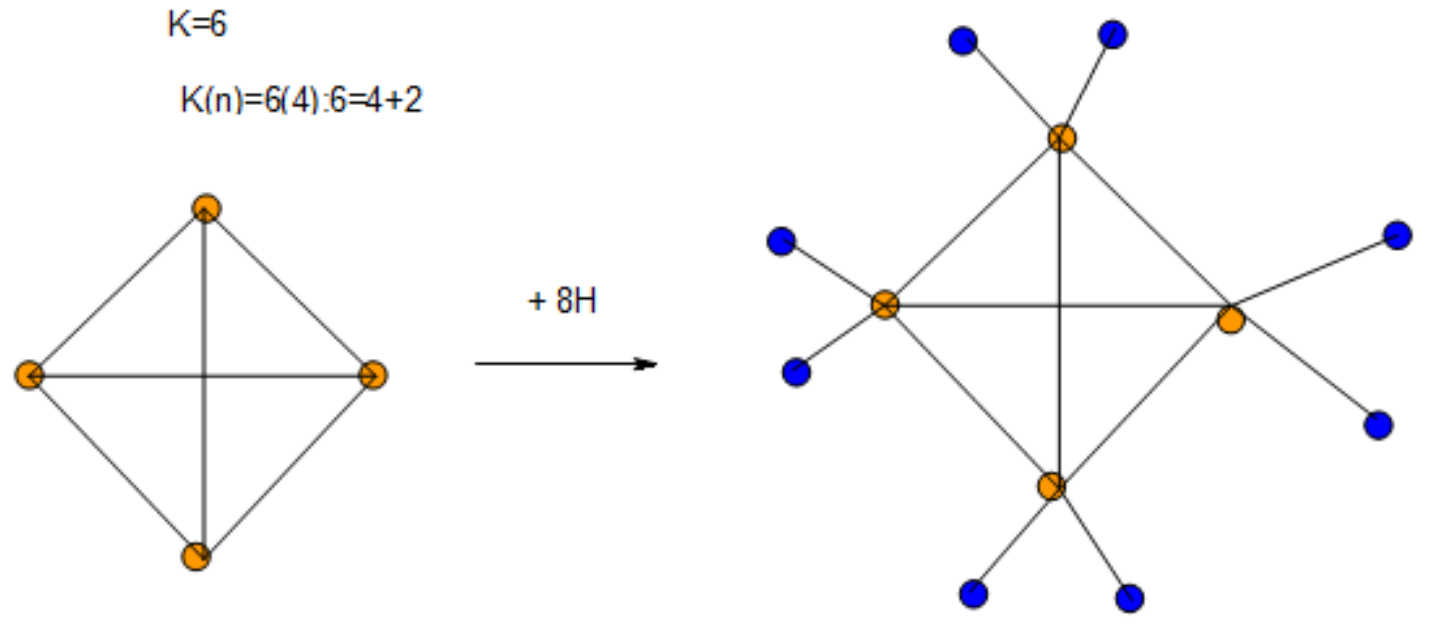

Tetrahedral skeletal shape

Figure 31. Isomeric graphical structure of $\mathrm{B}_{4} \mathrm{H}_{8}$ 
13. $\left.\mathrm{B}_{3} \mathrm{H}_{9}\{\mathrm{~K}=3] 2.5\right]-9[0.5=3, \mathrm{n}=3$

$\mathrm{K}(\mathrm{n})=3(3)$

$6-3=3$

$\mathrm{S}=4 \mathrm{n}+6$ (arachno)

$\mathrm{K}=2 \mathrm{n}-3$

$\mathrm{Kp}=\mathrm{C}^{-2} \mathrm{C}[\mathrm{M} 5]$

$\mathrm{K}^{*}=\mathrm{C}^{-2}+\mathrm{D}^{5}$

$\mathrm{y}=-2, \mathrm{z}=5$

$\mathrm{VE} 0=2 \mathrm{z}+2=2[5]+2=12$

$\mathrm{VEDz}=4 \mathrm{z}+2=4[5]+2=22$

$\mathrm{VE}=\mathrm{VE} 0+2 \mathrm{n}=12+2[3]=12+6=18$

$\mathrm{VE}=4 \mathrm{n}+6=4[3]+6=12+6=18$

$\mathrm{VF}=3[3]+9=9+9=18$

$\mathrm{VE}=\mathrm{VEDz}+2 \mathrm{y}=22+2[-2]=18$

$\mathrm{VE}=8 \mathrm{n}-2 \mathrm{~K}=8[3]-2[3]=24-6=18$

$\mathrm{VE}=\mathrm{VE} 0+2 \mathrm{y}+2 \mathrm{z}=12+2[-2]+2[5]=12-4+10=18$

$\mathrm{VE}=2 \mathrm{y}+4 \mathrm{z}+2=2[-2]+4[5]+2=22-4=18$

$\mathrm{K}=3$

$\mathrm{B}(\mathrm{K}=2.5, \mathrm{~V})$
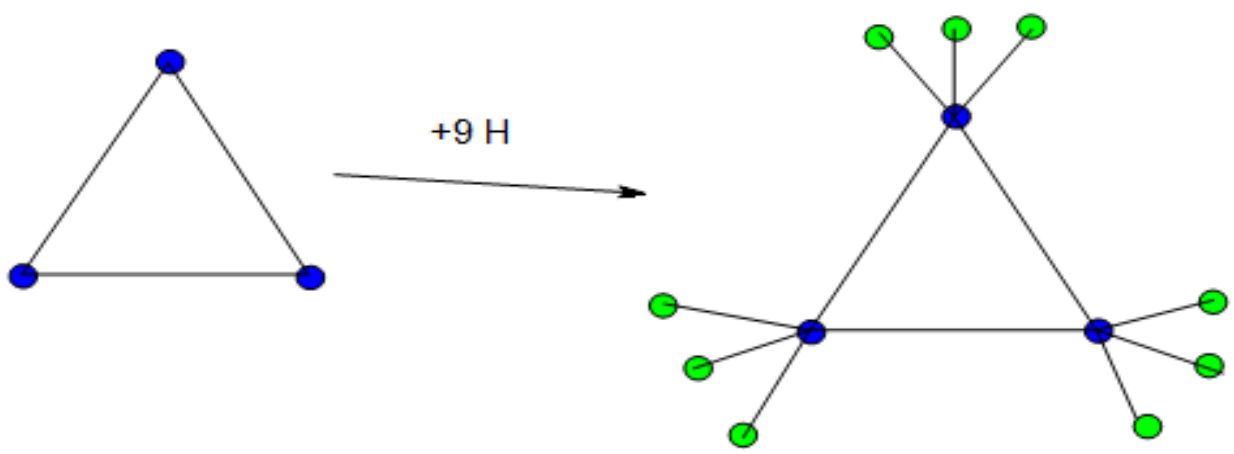

Figure 32. Isomeric graphical structure of $\mathrm{B}_{3} \mathrm{H}_{9}$

\section{$D^{6}$ SERIES}

14. $\mathrm{F}=\mathrm{B}_{6} \mathrm{H}_{6}{ }^{2-}: \mathrm{K}=6[2.5]-6[0.5]-2[0.5]=11, \mathrm{n}=6$

$\mathrm{K}(\mathrm{n})=11(6)$

$12-11=1$

$\mathrm{S}=4 \mathrm{n}+2$ (closo)

$\mathrm{K}=2 \mathrm{n}-1$

$\mathrm{Kp}=\mathrm{C}^{0} \mathrm{C}[\mathrm{M} 6]$

$\mathrm{K}^{*}=\mathrm{C}^{0}+\mathrm{D}^{6}$

$\mathrm{y}=0, \mathrm{z}=6$

$\mathrm{VE} 0=2 \mathrm{z}+2=2[6]+2=14$

$\mathrm{VED} z=4 \mathrm{z}+2=4[6]+2=26$

$\mathrm{VE}=8 \mathrm{n}-2 \mathrm{~K}=8[6]-2[11]=48-22=26$ 
$\mathrm{VE}=4 \mathrm{n}+2=4[6]+2=26$

$\mathrm{VE}=6[3]+6+2=18+6+2=26$

$\mathrm{VE}=\mathrm{VEDz}+2 \mathrm{y}=26+2[0]=26$

$\mathrm{VE}=2 \mathrm{y}+4 \mathrm{z}+2=2[0]+4[6]+2=26$

$\mathrm{VE}=\mathrm{VE} 0+2 \mathrm{y}+2 \mathrm{z}=14+2[0]+2[6]=14+12=26$

$\mathrm{VF}=6[3]+6+2=18+6+2=26$

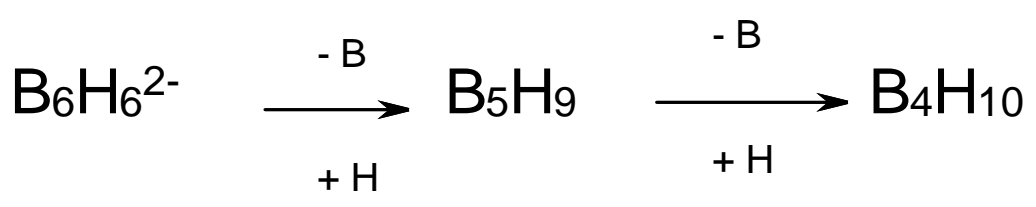

15. $\mathrm{B}_{5} \mathrm{H}_{9}: \mathrm{K}=5[2.5]-9[0.5]=8, \mathrm{n}=5$

$\mathrm{K}(\mathrm{n})=8(5)$

$10-8=2$

$\mathrm{S}=4 \mathrm{n}+4$

$\mathrm{K}=2 \mathrm{n}-2$

$\mathrm{Kp}=\mathrm{C}^{-1} \mathrm{C}[\mathrm{M} 6]$

$\mathrm{K}^{*}=\mathrm{C}^{-1}+\mathrm{D}^{6}$

$\mathrm{y}=-1, \mathrm{z}=6$

$\mathrm{VE} 0=2 \mathrm{z}+2=2[6]+2=14$

$\mathrm{VEDz}=4 \mathrm{z}+2=4[6]+2=26$

$\mathrm{VE}=8 \mathrm{n}-2 \mathrm{~K}=8[5]-2[8]=40-16=24$

$\mathrm{VE}=4 \mathrm{n}+4=4[5]+4=24$

$\mathrm{VE}=\mathrm{VE} 0+2 \mathrm{n}=14+2[5]=24$

$\mathrm{VE}=\mathrm{VE} 0+2 \mathrm{y}+2 \mathrm{z}=14+2[-1]+2[6]=14-2+12=24$

$\mathrm{VE}=2 \mathrm{y}+4 \mathrm{z}+2=2[-1]+4[6]+2=-2+24+2=24$

$\mathrm{K}=8$
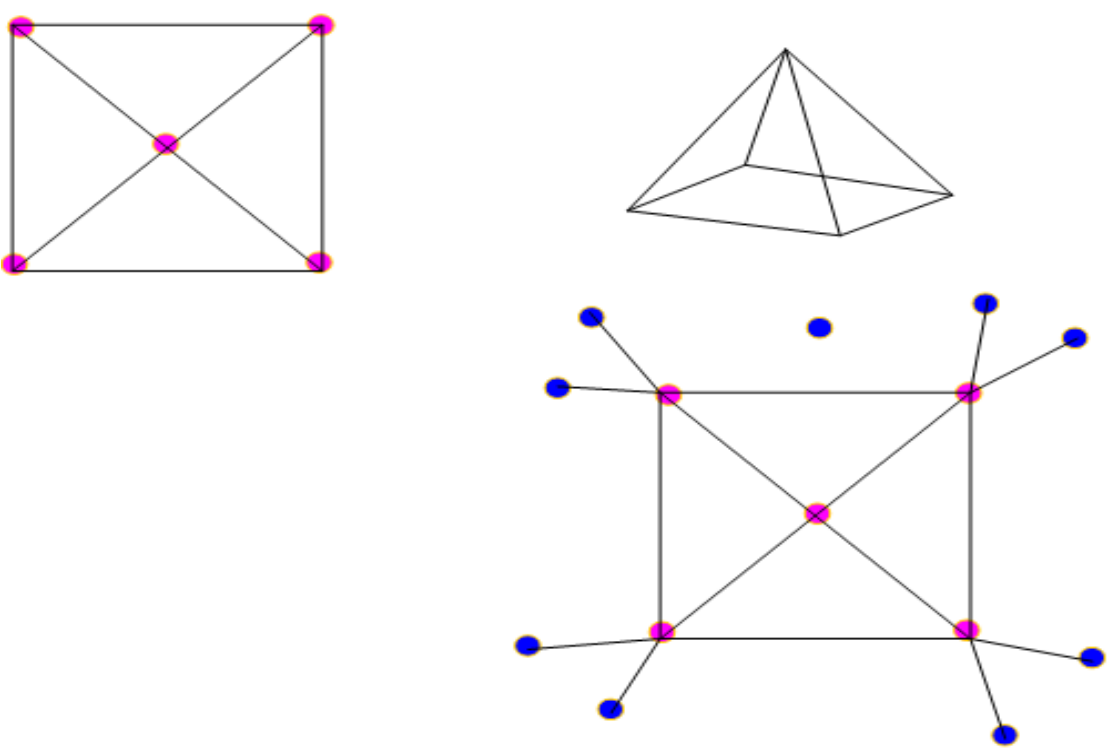

Figure 33. Isomeric graphical structure of $\mathrm{B}_{5} \mathrm{H}_{9}$ 
16. $\mathrm{B}_{4} \mathrm{H}_{10}: \mathrm{K}=4[2.5]-5=5, \mathrm{n}=4$

$\mathrm{K}(\mathrm{n})=5(4)$

$8-5=3$

$\mathrm{S}=4 \mathrm{n}+6$

$\mathrm{K}=2 \mathrm{n}-3$

$\mathrm{Kp}=\mathrm{C}^{-2}[\mathrm{M} 6]$

$\mathrm{K}^{*}=\mathrm{C}^{-2}+\mathrm{D}^{6}$

$\mathrm{y}=-2, \mathrm{z}=6$

$\mathrm{VE} 0=2 \mathrm{z}+2=2[6]+2=14$

$\mathrm{VEDz}=4 \mathrm{z}+2=4[6]+2=26$

$\mathrm{VE}=\mathrm{VE} 0+2 \mathrm{n}=14+2[4]=22$;

$\mathrm{S}=4 \mathrm{n}+6=4[4]+6=22$

$\mathrm{VE}=8 \mathrm{n}-2 \mathrm{~K}=8[4]-2[5]=32-10=22$

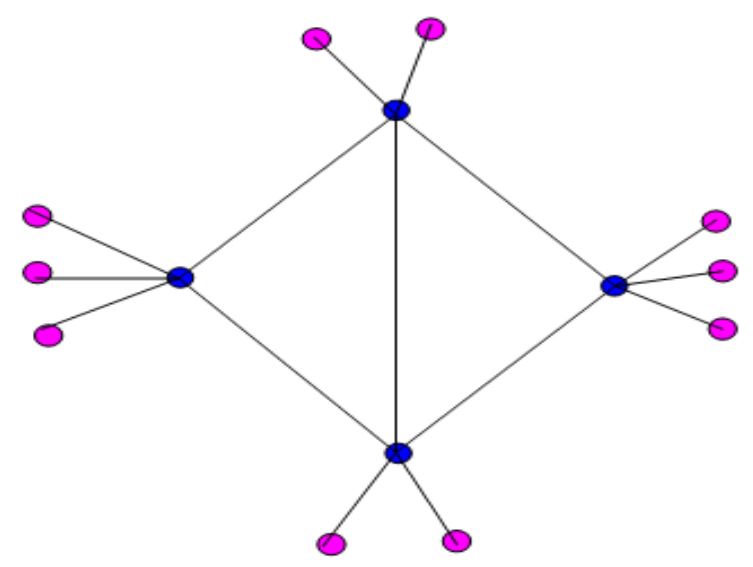

Figure 34. Isomeric graphical structure of $\mathrm{B}_{4} \mathrm{H}_{10}$

The structures in Figures 18 and 4 are skeletal isomers.

\section{$D^{7}$ SERIES}
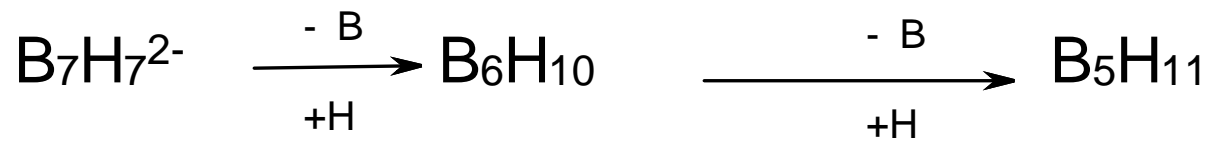

17. $\mathrm{B}_{7} \mathrm{H}_{7}^{2-}: \mathrm{K}=7[2.5]-7[0.5]-2[0.5]=13, \mathrm{n}=7$

$\mathrm{K}(\mathrm{n})=13(7)$

$14-13=1$

$\mathrm{S}=4 \mathrm{n}+2($ closo $)$

$\mathrm{K}=2 \mathrm{n}-1$

$\mathrm{Kp}=\mathrm{C}^{0} \mathrm{C}[\mathrm{M} 7]$

$\mathrm{K}^{*}=\mathrm{C}^{0}+\mathrm{D}^{7}$

$\mathrm{y}=0, \mathrm{z}=7$

$\mathrm{VE} 0=2 \mathrm{z}+2=2[7]+2=16$

$\mathrm{VEDz}=4 \mathrm{z}+2=4[7]+2=30$

$\mathrm{VE}=8 \mathrm{n}-2 \mathrm{~K}=8[7]-2[13]=56-26=30$

$\mathrm{VE}=4 \mathrm{n}+2=4[7]+2=30$ 
$\mathrm{VE}=\mathrm{VE} 0+2 \mathrm{n}=16+2[7]=16+14=30$

$\mathrm{VF}=7[3]+7+2=21+9=30$

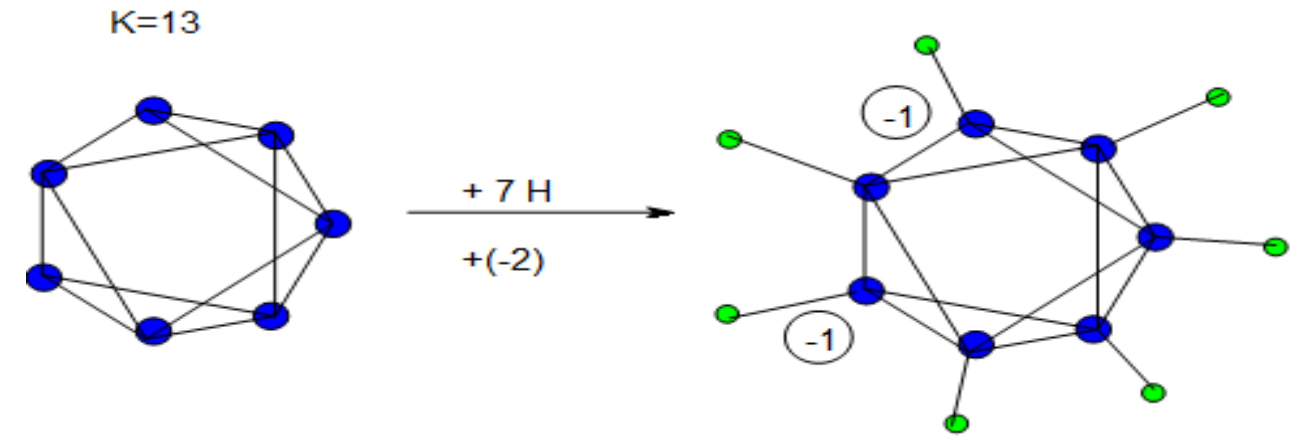

Figure 35. Isomeric graphical structure of $\mathrm{B}_{7} \mathrm{H}_{7}{ }^{2-}$

18. $\mathrm{B}_{6} \mathrm{H}_{10}: \mathrm{K}=6[2.5]-10[0.5]=10, \mathrm{n}=6$

$\mathrm{K}(\mathrm{n})=10(6)$

$12-10=2$

$S=4 n+4$ (nido)

$\mathrm{K}=2 \mathrm{n}-2$

$\mathrm{Kp}=\mathrm{C}^{-1} \mathrm{C}[\mathrm{M} 7]$

$\mathrm{K}^{*}=\mathrm{C}^{-1}+\mathrm{D}^{7}$

$\mathrm{y}=-1, \mathrm{z}=7$

$\mathrm{VE} 0=2 \mathrm{z}+2=2[7]+2=16$

$\mathrm{VE}=\mathrm{VE} 0+2 \mathrm{n}=16+2[6]=16+12=28$

$\mathrm{VE}=4 \mathrm{n}+4=4[6]+4=24+4=28$

$\mathrm{VE}=8 \mathrm{n}-2 \mathrm{~K}=8[6]-2[10]=48-20=28$

$\mathrm{VF}=6[3]+10=28$
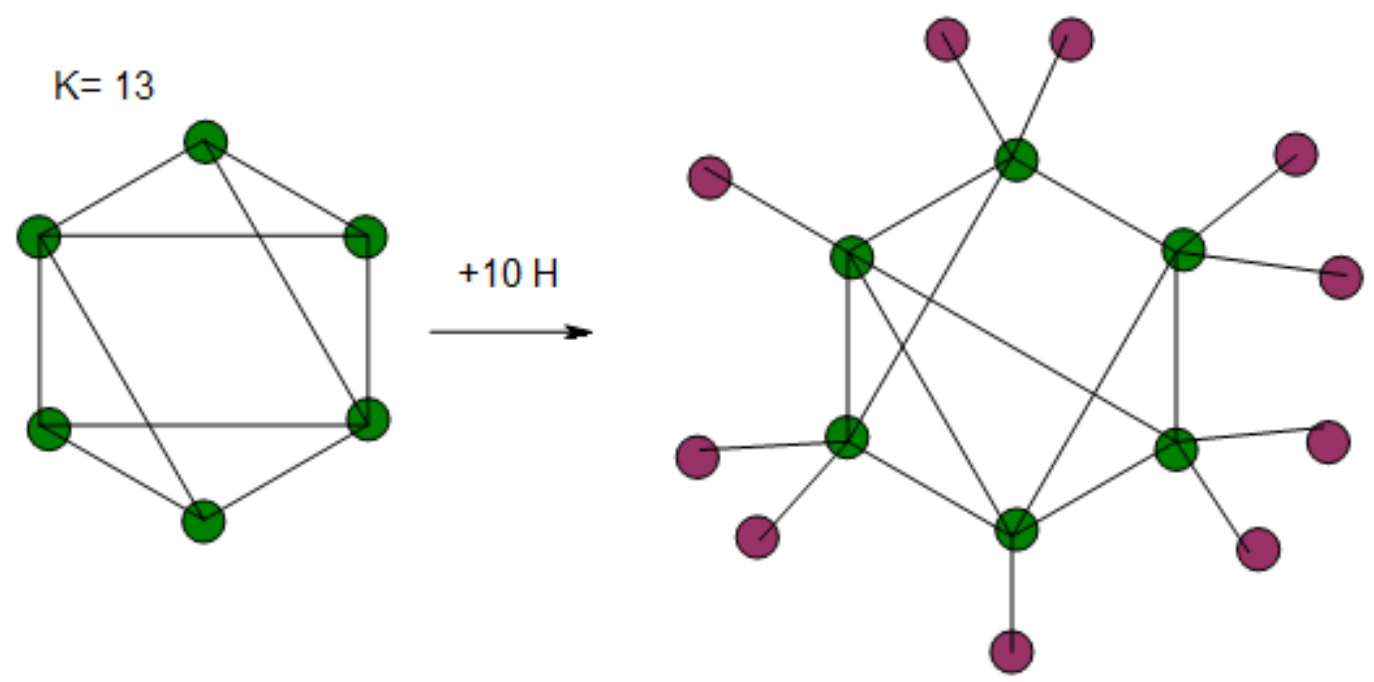

Figure 36. Isomeric graphical structure of $\mathrm{B}_{6} \mathrm{H}_{10}$

19. $\mathrm{B}_{5} \mathrm{H}_{11}: \mathrm{K}=5[2.5]-11[0.5]=7, \mathrm{n}=5$

$\mathrm{K}(\mathrm{n})=7(5)$

$10-7=3$ 
$\mathrm{S}=4 \mathrm{n}+6$ (arachno)

$\mathrm{K}=2 \mathrm{n}-3$

$\mathrm{Kp}=\mathrm{C}^{-2} \mathrm{C}[\mathrm{M} 7]$

$\mathrm{K}^{*}=\mathrm{C}^{-2}+\mathrm{D}^{7}$

$\mathrm{y}=-2, \mathrm{z}=7$

$\mathrm{VE} 0=2 \mathrm{z}+2=2[7]+2=16$

$\mathrm{VE}=\mathrm{VE} 0+2 \mathrm{n}=16+2[5]=16+10=26$

$\mathrm{VE}=8 \mathrm{n}-2 \mathrm{~K}=8[5]-2[7]=40-14=26$

$\mathrm{VE}=4 \mathrm{n}+6=4[5]+6=26$

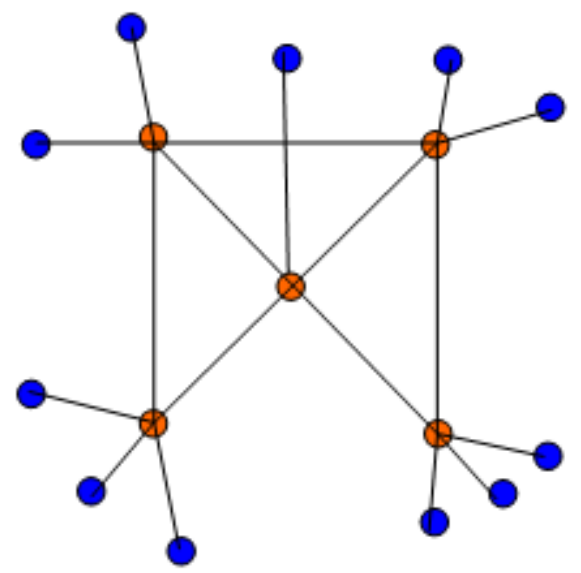

Figure 37. Isomeric graphical structure of $\mathrm{B}_{5} \mathrm{H}_{11}$

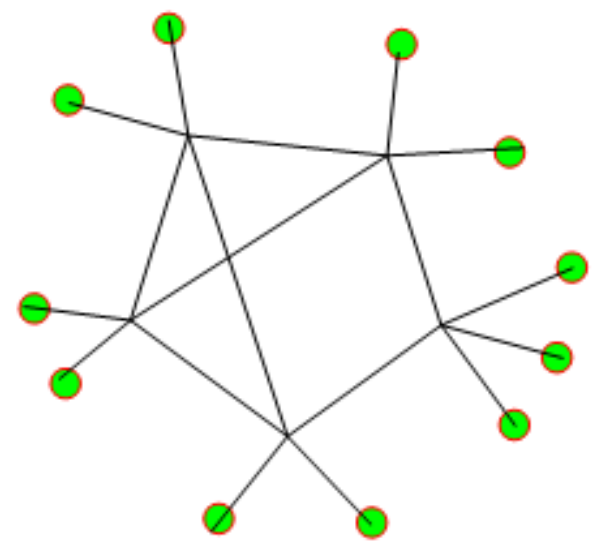

Figure 38. Isomeric graphical structure of $\mathrm{B}_{5} \mathrm{H}_{11}$

\section{$D^{8}$ SERIES}

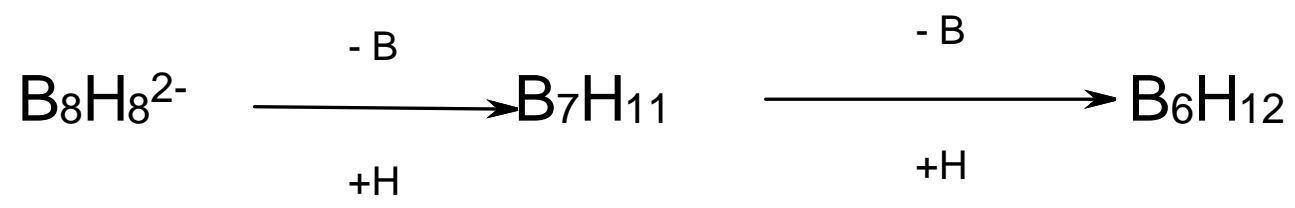

20. $\mathrm{B}_{8} \mathrm{H}_{8}{ }^{2-}: \mathrm{K}=8[2.5]-8[0.5]-2[05]=15, \mathrm{n}=8$

$\mathrm{K}(\mathrm{n})=15(8)$

$16-15=1$ 
$\mathrm{S}=4 \mathrm{n}+2($ closo $)$

$\mathrm{K}=2 \mathrm{n}-1$

$\mathrm{Kp}=\mathrm{C}^{0} \mathrm{C}[\mathrm{M} 8]$

$\mathrm{K}^{*}=\mathrm{C}^{0}+\mathrm{D}^{8}$

$\mathrm{y}=0, \mathrm{z}=8$

$\mathrm{VE} 0=2 \mathrm{z}+2=2[8]+2=18$

$\mathrm{VE}=\mathrm{VE} 0+2 \mathrm{n}=18+2[8]=18+16=34$

$\mathrm{VE}=4 \mathrm{n}+2=4[8]+2=34$

$\mathrm{VF}=8[3]+8+2=24+10=34$

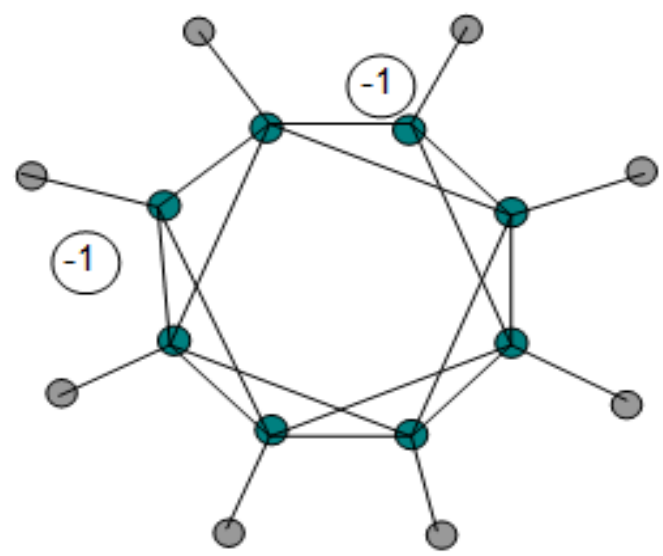

Figure 39. Isomeric graphical structure of $\mathrm{B}_{8} \mathrm{H}_{8}{ }^{2-}$

21. $\mathrm{B}_{7} \mathrm{H}_{11}: \mathrm{K}=7[2.5]-11[0.5]=12, \mathrm{n}=7$

$\mathrm{K}(\mathrm{n})=12(7)$

$14-12=2$

$S=4 n+4$ (nido)

$\mathrm{K}=2 \mathrm{n}-2$

$\mathrm{Kp}=\mathrm{C}^{-1} \mathrm{C}[\mathrm{M} 8]$

$\mathrm{K}^{*}=\mathrm{C}^{-1}+\mathrm{D}^{8}$

$\mathrm{y}=-1, \mathrm{z}=8$

$\mathrm{VE} 0=2 \mathrm{z}+2=2[8]+2=18$

$\mathrm{VE}=4 \mathrm{n}+4=4[7]+4=32$

$\mathrm{VE}=\mathrm{VE} 0+2 \mathrm{n}=18+2[7]=18+14=32$

$\mathrm{VF}=7[3]=11=21+11=32$ 


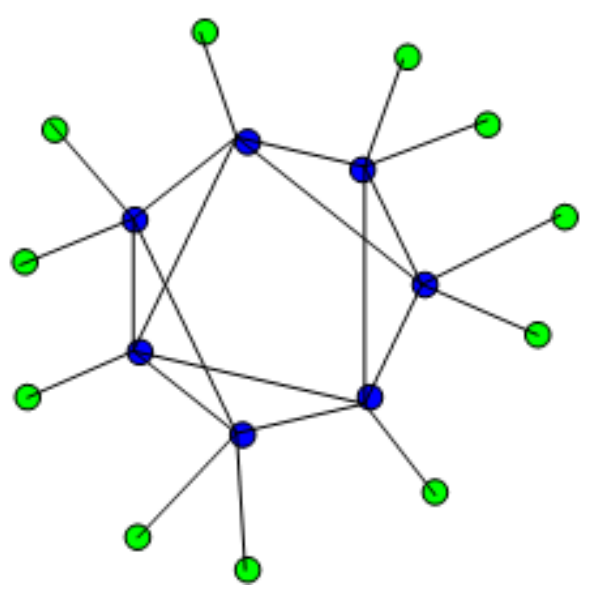

Figure 40. Isomeric graphical structure of $\mathrm{B}_{7} \mathrm{H}_{11}$

22. $\mathrm{B}_{6} \mathrm{H}_{12}: \mathrm{K}=6[2.5]-6=9, \mathrm{n}=6$

$\mathrm{K}(\mathrm{n})=9(6)$

$12-9=3$

$\mathrm{S}=4 \mathrm{n}+6$ (arachno)

$\mathrm{K}=2 \mathrm{n}-3$

$\mathrm{Kp}=\mathrm{C}^{-2} \mathrm{C}[\mathrm{M} 8]$

$\mathrm{K}^{*}=\mathrm{C}^{-2}+\mathrm{D}^{8}$

$\mathrm{y}=-2, \mathrm{z}=8$

$\mathrm{VE} 0=2 \mathrm{z}+2=2[8]+2=18$

$\mathrm{VE}=\mathrm{VE} 0+2 \mathrm{n}=18+2[6]=18+12=30$

$\mathrm{VE}=8 \mathrm{n}-2 \mathrm{~K}=8[6]-2[9]=48-18=30$

$\mathrm{VE}=4 \mathrm{n}+6=4[6]+6=24+6=30$
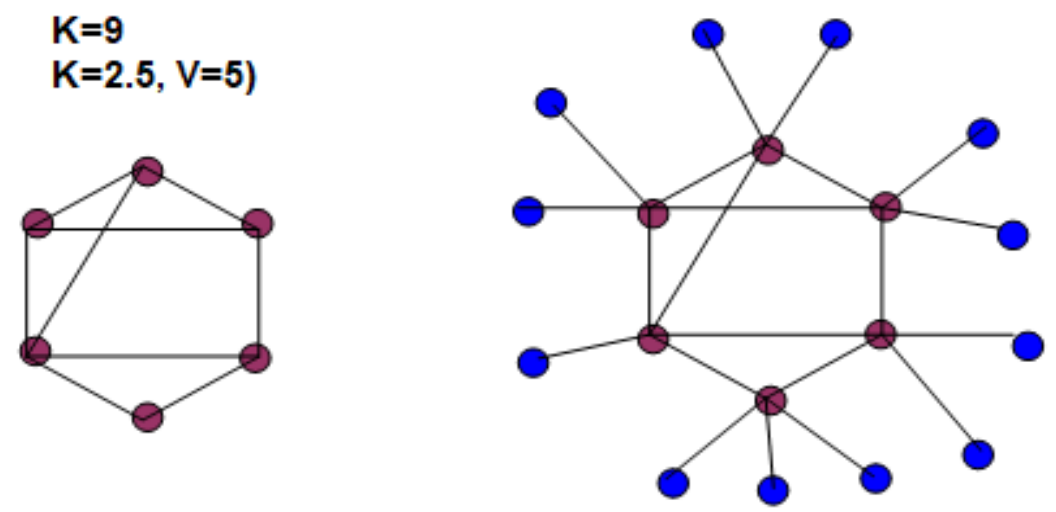

Figure 41. Isomeric graphical structure of $\mathrm{B}_{6} \mathrm{H}_{12}$

\section{D' SERIES}

$$
\mathrm{B}_{9} \mathrm{H}_{9}^{2-} \longrightarrow \mathrm{B}_{8} \mathrm{H}_{12} \longrightarrow \mathrm{B}_{7} \mathrm{H}_{13}
$$

23. $\mathrm{K}=9[2.5]-9[0.5]-2[0.5]=17, \mathrm{n}=9$

$\mathrm{K}(\mathrm{n})=17(9)$

$18-17=1$ 
$\mathrm{S}=4 \mathrm{n}+2$ (closo)

$\mathrm{K}=2 \mathrm{n}-1$

$\mathrm{Kp}=\mathrm{C}^{0} \mathrm{C}$ [M9]

$\mathrm{K}^{*}=\mathrm{C}^{0}+\mathrm{D}^{9}$

$\mathrm{y}=0, \mathrm{z}=9$

$\mathrm{VE} 0=2 \mathrm{z}+2=2[9]+2=20$

$\mathrm{VE}=\mathrm{VE} 0+2 \mathrm{n}=20+2[9]=38$

$\mathrm{VE}=4 \mathrm{n}+2=4[9]+2=38$

$\mathrm{VF}=9[3]+9+2=27+9+2=27+11=38$

$$
\mathrm{K}=17
$$
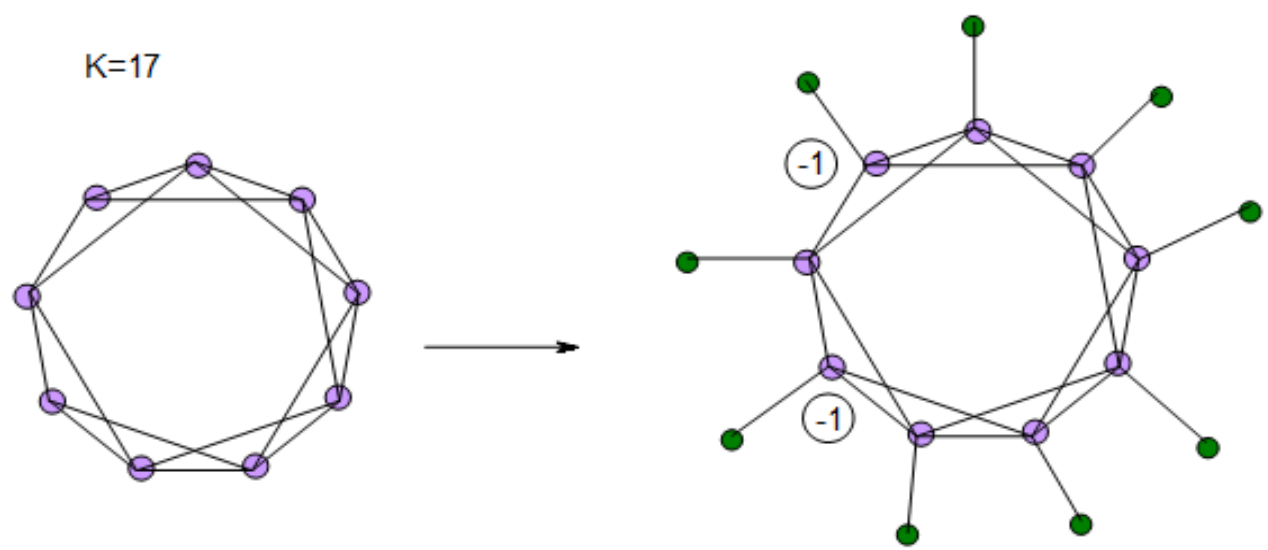

Figure 42. Isomeric graphical structure of $\mathrm{B}_{9} \mathrm{H}_{9}{ }^{2-}$

24. $\mathrm{B}_{8} \mathrm{H}_{12}: \mathrm{K}=8[2.5]-6=14, \mathrm{n}=8$

$\mathrm{K}(\mathrm{n})=14(8)$

$16-14=2$

$\mathrm{S}=4 \mathrm{n}+4$ (nido)

$\mathrm{K}=2 \mathrm{n}-2$

$\mathrm{Kp}=\mathrm{C}^{-1} \mathrm{C}[\mathrm{M} 9]$

$\mathrm{K}^{*}=\mathrm{C}^{-1}+\mathrm{D}^{9}$

$\mathrm{y}=-1, \mathrm{z}=9$

$\mathrm{VE} 0=2 \mathrm{z}+2=2[9]+2=20$

$\mathrm{VE}=\mathrm{VE} 0+2 \mathrm{n}=20+2[8]=20+16=36$

$\mathrm{VE}=8 \mathrm{n}-2 \mathrm{~K}=8[8]-2[14]=64-28=36$

$\mathrm{VE}=4 \mathrm{n}+4=4[8]+4=32+4=36$
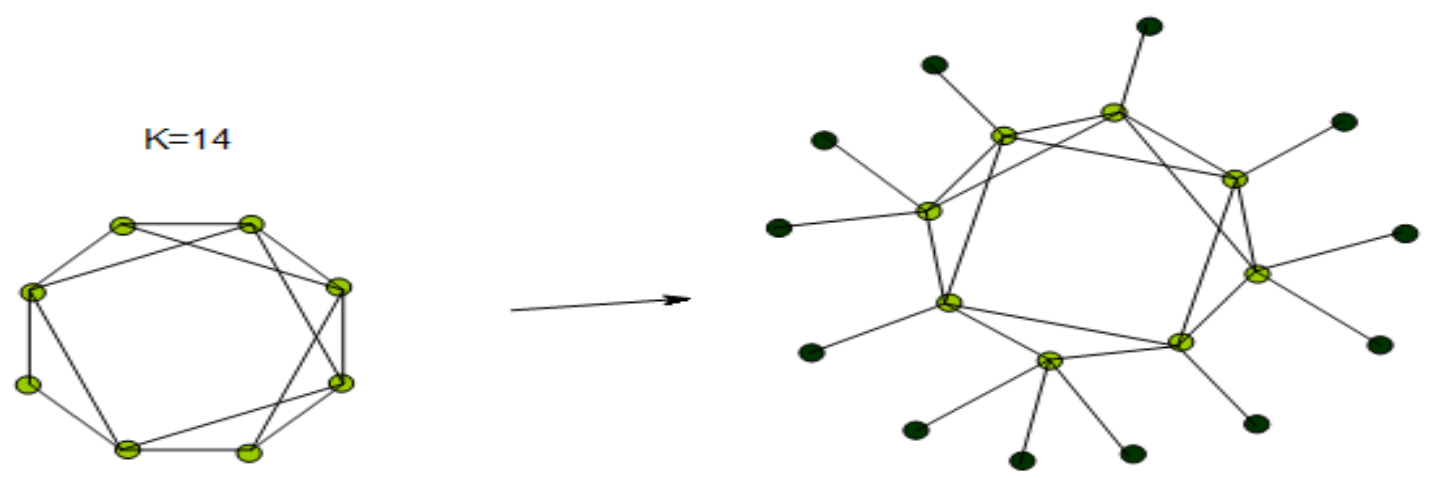

Figure 43. Isomeric graphical structure of $\mathrm{B}_{8} \mathrm{H}_{12}$ 
25. $\mathrm{B}_{7} \mathrm{H}_{13:} \mathrm{K}=7[2.5]-13[0.5]=11, \mathrm{n}=7$
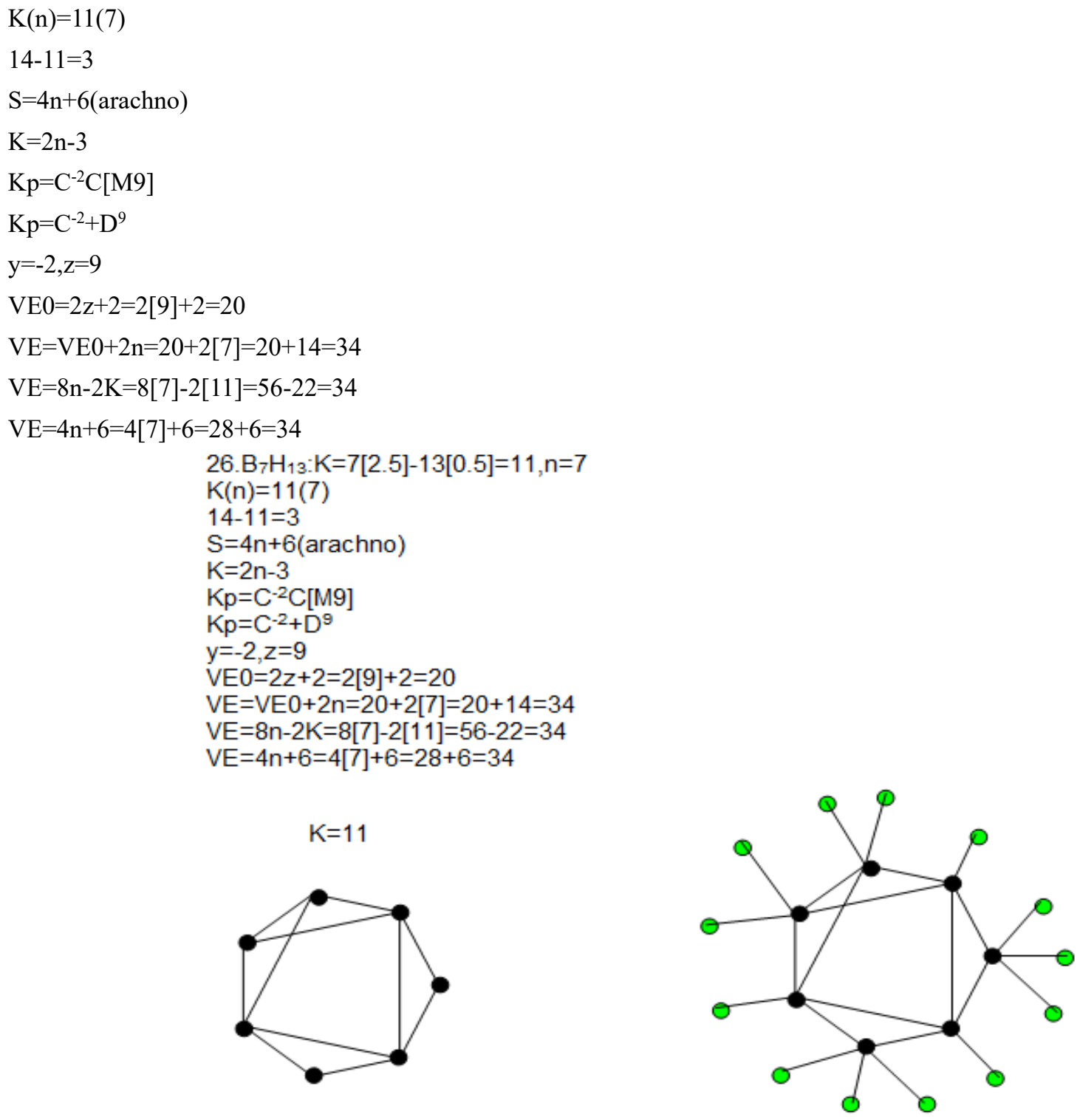

Figure 44. Isomeric graphical structure of $\mathrm{B}_{7} \mathrm{H}_{13}$

\section{$D^{10}$ SERIES}

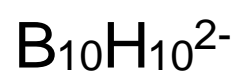

\section{$\mathrm{B}_{9} \mathrm{H}_{13}$}

27. $\mathrm{B}_{10} \mathrm{H}_{10}{ }^{2-}: \mathrm{K}=10[2.5]-5-1=19, \mathrm{n}=10$

$\mathrm{K}(\mathrm{n})=19(10)$

$20-19=1$

$\mathrm{S}=4 \mathrm{n}+2($ closo $)$

$\mathrm{K}=2 \mathrm{n}-1$

$\mathrm{Kp}=\mathrm{C}^{0} \mathrm{C}[\mathrm{M} 10]$

$\mathrm{K}^{*}=\mathrm{C}^{0}+\mathrm{D}^{10}$

$\mathrm{y}=0, \mathrm{z}=10$ 
$\mathrm{VE} 0=2 \mathrm{z}+2=2[10]+2=22$

$\mathrm{VE}=\mathrm{VE} 0+2 \mathrm{n}=22+2[10]=22+20=42$

$\mathrm{VE}=8 \mathrm{n}-2 \mathrm{~K}=8[10]-2[19]=80-38=42$

$\mathrm{VE}=4 \mathrm{n}+2=4[10]+2=42$

$\mathrm{VF}=10[3]+10+2=42$
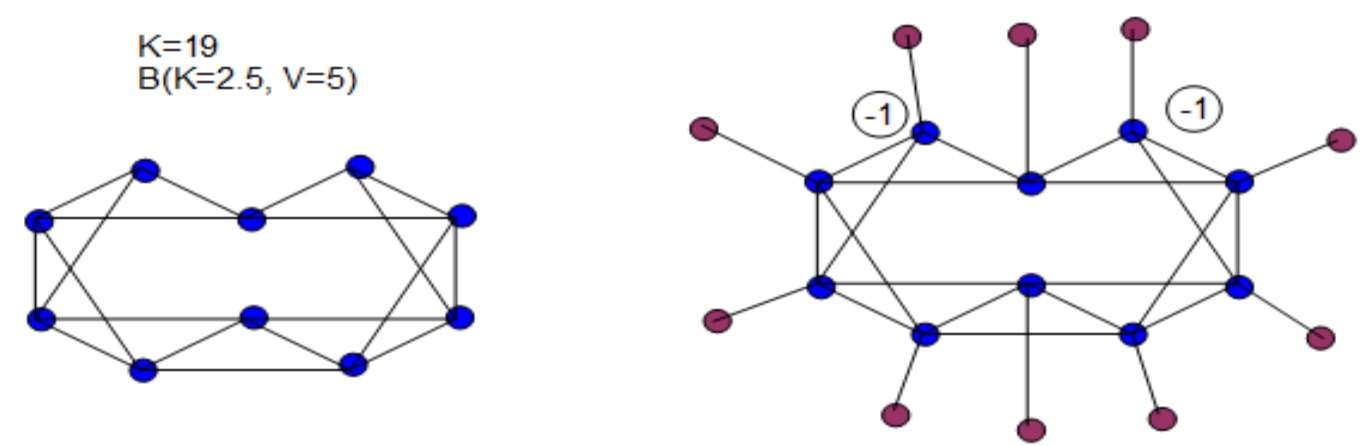

Figure 45. Isomeric graphical structure of $\mathrm{B}_{10} \mathrm{H}_{10}{ }^{2-}$

$28 \mathrm{~B}_{9} \mathrm{H}_{13}: \mathrm{K}=9[2.5]-13[0.5=16, \mathrm{n}=9$

$\mathrm{K}(\mathrm{n})=16(9)$

$18-16=2$

$\mathrm{S}=4 \mathrm{n}+4$ (nido)

$\mathrm{K}=2 \mathrm{n}-2$

$\mathrm{Kp}=\mathrm{C}^{-1} \mathrm{C}[\mathrm{M} 10]$

$\mathrm{K}^{*}=\mathrm{C}^{-1}+\mathrm{D}^{10}$

$\mathrm{y}=-1, \mathrm{z}=10$

$\mathrm{VE} 0=2 \mathrm{z}+2=2[10]+2=22$

$\mathrm{VE}=\mathrm{VE} 0+2 \mathrm{n}=22+2[9]=22+18=40$

$\mathrm{VE}=8 \mathrm{n}-2 \mathrm{~K}=8[9]-2[16]=72-32=40$

$\mathrm{VE}=4 \mathrm{n}+4=4[9]+4=36+4=40$

$\mathrm{VF}=9[3]+13=27+13=40$
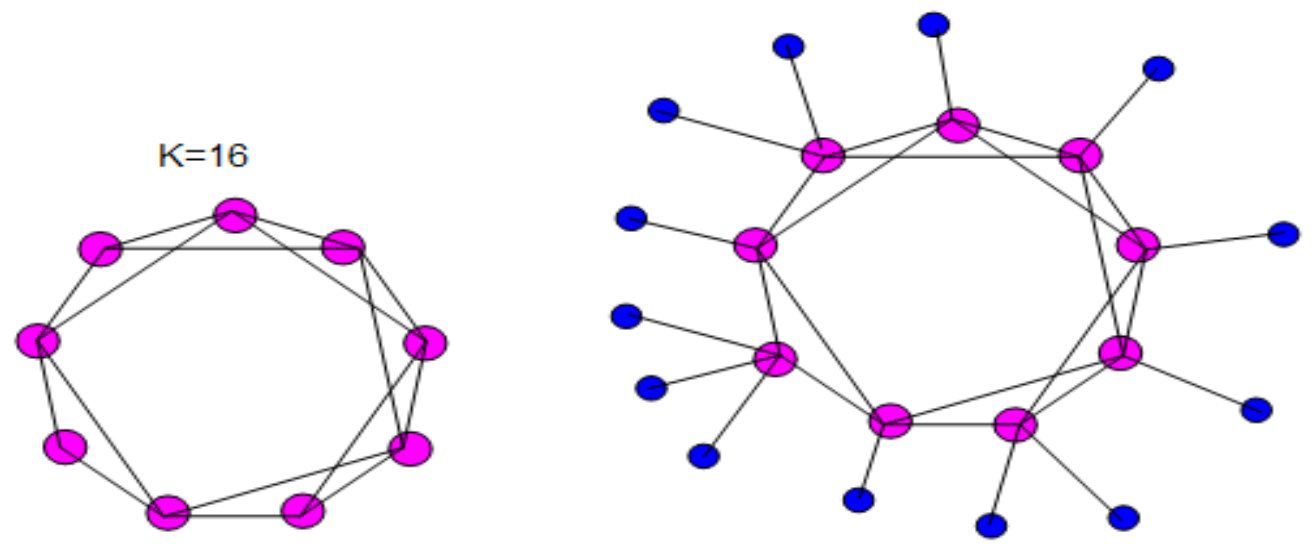

Figure 46. Isomeric graphical structure of $\mathrm{B}_{9} \mathrm{H}_{13}$

\section{$D^{11}$ SERIES}

29. $\mathrm{B}_{11} \mathrm{H}_{11^{2}}{ }^{-}: \mathrm{K}=11[2.5]-5.5-1=21, \mathrm{n}=11$

$\mathrm{K}(\mathrm{n})=21(11)$

$22-21=1$ 
$\mathrm{S}=4 \mathrm{n}+2($ closo $)$

$\mathrm{K}=2 \mathrm{n}-1$

$\mathrm{Kp}=\mathrm{C}^{0} \mathrm{C}[\mathrm{M} 11]$

$\mathrm{K}^{*}=\mathrm{C}^{0}+\mathrm{D}^{11}$

$\mathrm{y}=0, \mathrm{z}=11$

$\mathrm{VE} 0=2 \mathrm{z}+2=2[11]+2=24$

$\mathrm{VE}=\mathrm{VE} 0+2 \mathrm{n}=24+2[11]=24+22=46$

$\mathrm{VE}=8 \mathrm{n}-2 \mathrm{~K}=8[11]-2[21]=88-42=46$

$\mathrm{VE}=4 \mathrm{n}+2=4[11]+2=46$

$\mathrm{VF}=11[3]+11+2=33+13=46$
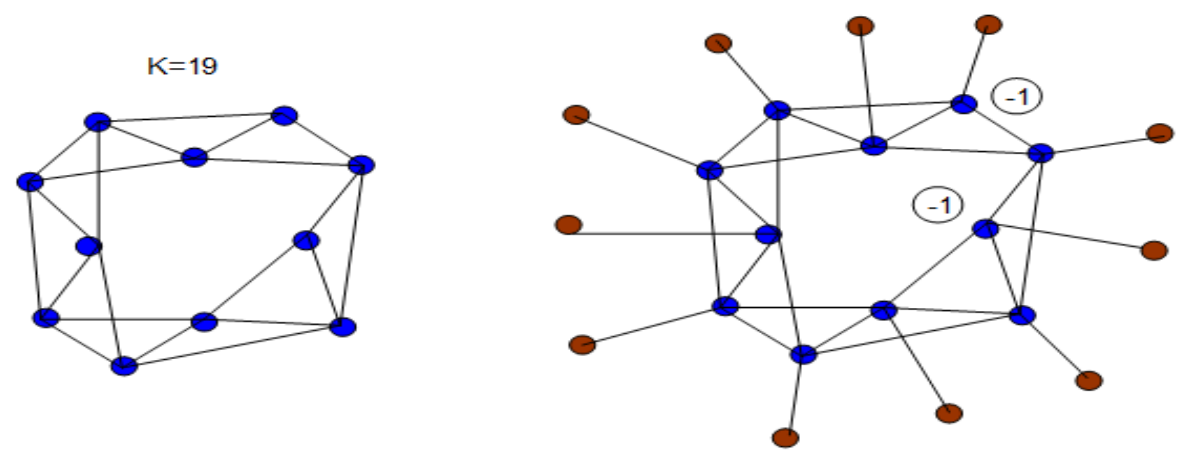

Figure 47. Isomeric graphical structure of $\mathrm{B}_{11} \mathrm{H}_{11}{ }^{2-}$

30. $\mathrm{B}_{10} \mathrm{H}_{14}: \mathrm{K}=10[2.5]-7=18, \mathrm{n}=10$

$\mathrm{K}(\mathrm{n})=18(10)$

$20-18=2$

$\mathrm{S}=4 \mathrm{n}+4$ (nido)

$\mathrm{K}=2 \mathrm{n}-2$

$\mathrm{Kp}=\mathrm{C}^{-1} \mathrm{C}[\mathrm{M} 11]$

$\mathrm{K}^{*}=\mathrm{C}^{-1}+\mathrm{D}^{11}$

$\mathrm{y}=-1, \mathrm{z}=11$

$\mathrm{VE} 0=2 \mathrm{z}+2=2[11]+2=24$

$\mathrm{VE}=\mathrm{VE} 0+2 \mathrm{n}=24+2[10]=24+20=44$

$\mathrm{VE}=8 \mathrm{n}-2 \mathrm{~K}=8[10]-2[18]=80-36=44$

$\mathrm{VE}=4 \mathrm{n}+4=4[10]+4=44$

$\mathrm{VF}=10[3]+14=44$

$\mathrm{K}=18$

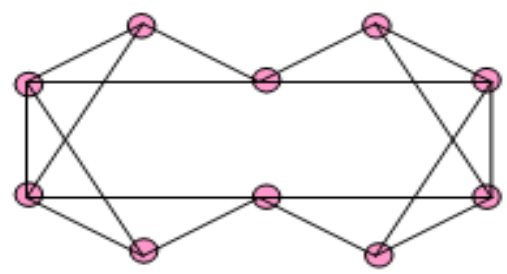

$\mathrm{B}(\mathrm{K}=2.5, \mathrm{~V}=5)$

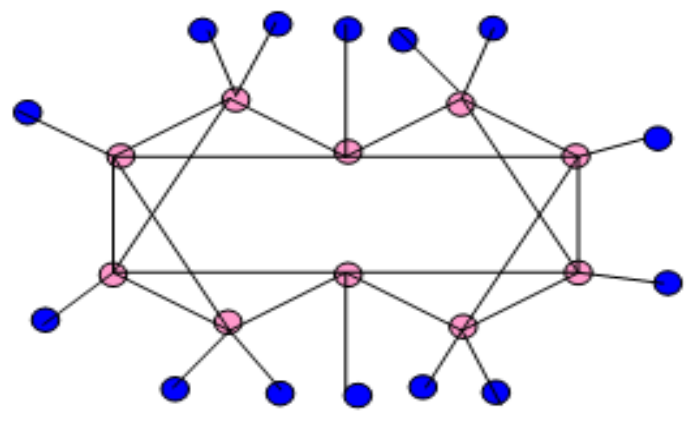

Figure 48. Isomeric graphical structure of $\mathrm{B}_{10} \mathrm{H}_{14}$ 

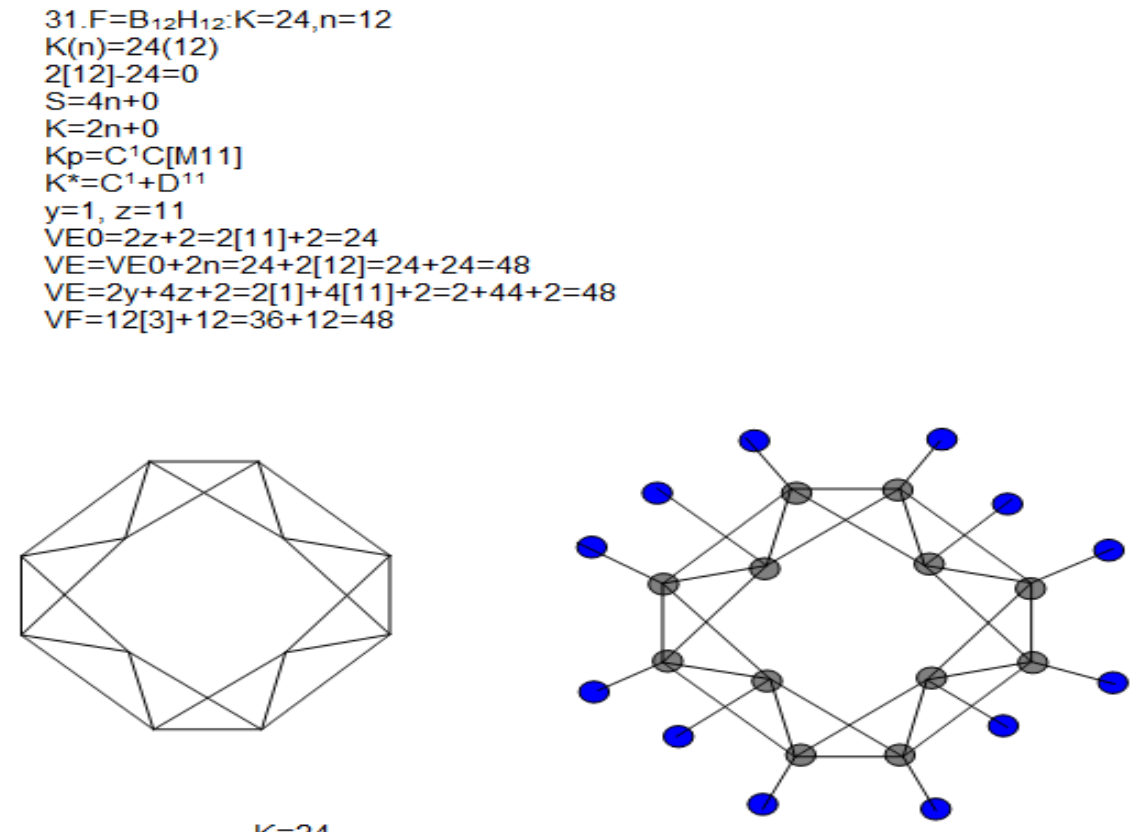

$\mathrm{K}=24$

$24=12+12$

Figure 49. Isomeric graphical structure of $\mathrm{B}_{12} \mathrm{H}_{12}$

\section{$D^{12}$ SERIES}

32. $\mathrm{B}_{12} \mathrm{H}_{12}{ }^{2-}: \mathrm{K}=12[2.5]-6-1=23, \mathrm{n}=12$

$\mathrm{K}(\mathrm{n})=23(12)$

$24-23=1$

$\mathrm{S}=4 \mathrm{n}+2$ (closo)

$\mathrm{K}=2 \mathrm{n}-1$

$\mathrm{Kp}=\mathrm{C}^{0} \mathrm{C}[\mathrm{M} 12]$

$\mathrm{K}^{*}=\mathrm{C}^{0}+\mathrm{D}^{12}$

$\mathrm{y}=0, \mathrm{z}=12$

$\mathrm{VE} 0=2 \mathrm{z}+2=2[12]+2=26$

$\mathrm{VE}=\mathrm{VE} 0+2 \mathrm{n}=26+2[12]=26+24=50$

$\mathrm{VE}=8 \mathrm{n}-2 \mathrm{~K}=8[12]-2[23]=96-46=50$

$\mathrm{VE}=4 \mathrm{n}+2=4[12]+2=50$

$\mathrm{VF}=12[3]+12+2=36+14=50$
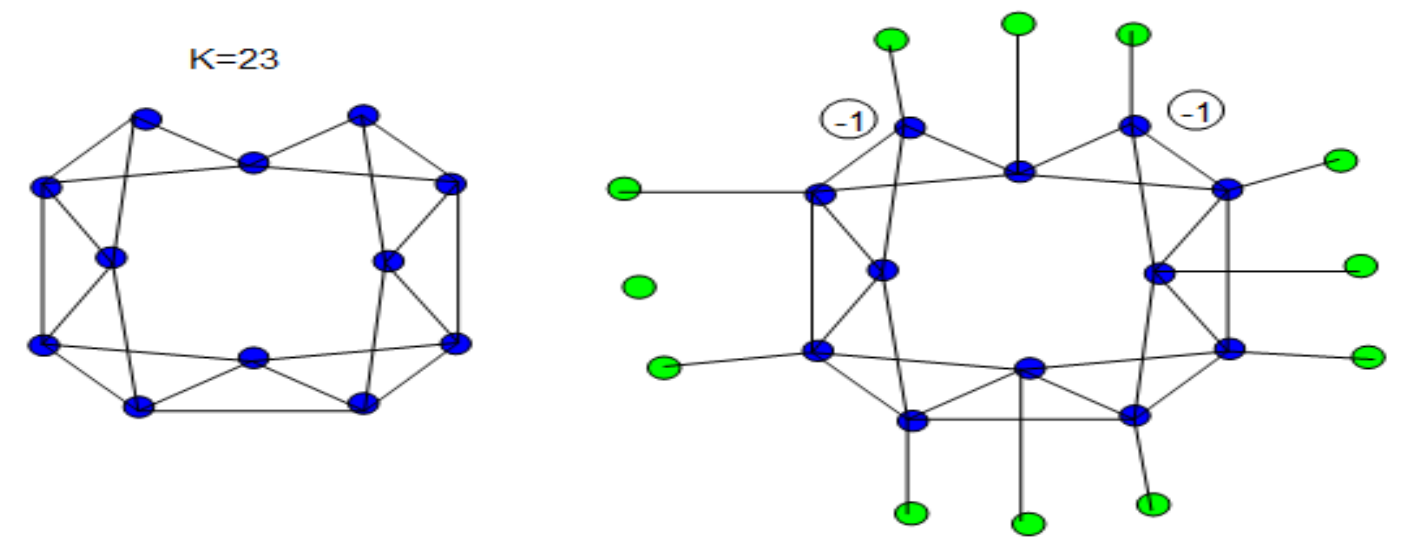

Figure 50. Isomeric graphical structure of $\mathrm{B}_{12} \mathrm{H}_{12}{ }^{2-}$ 
33. $\mathrm{B}_{11} \mathrm{H}_{15}: \mathrm{K}=11[2.5]-15[0.5]=20, \mathrm{n}=11$

$\mathrm{K}(\mathrm{n})=20(11)$

$22-20=2$

$\mathrm{S}=4 \mathrm{n}+4$ (nido)

$\mathrm{K}=2 \mathrm{n}-2$

$\mathrm{Kp}=\mathrm{C}^{-1} \mathrm{C}[\mathrm{M} 12]$

$\mathrm{K}^{*}=\mathrm{C}^{-1}+\mathrm{D}^{12}$

$\mathrm{y}=-1, \mathrm{z}=12$

$\mathrm{VE} 0=2 \mathrm{z}+2=2[12]+2=26$

$\mathrm{VE}=\mathrm{VE} 0+2 \mathrm{n}=26+2[11]=26+22=48$

$\mathrm{VE}=8 \mathrm{n}-2 \mathrm{~K}=8[11]-2[20]=88-40=48$

$\mathrm{VE}=4 \mathrm{n}+4=4[11]+4=44+4=48$

$\mathrm{VF}=11[3]+15=33+15=48$
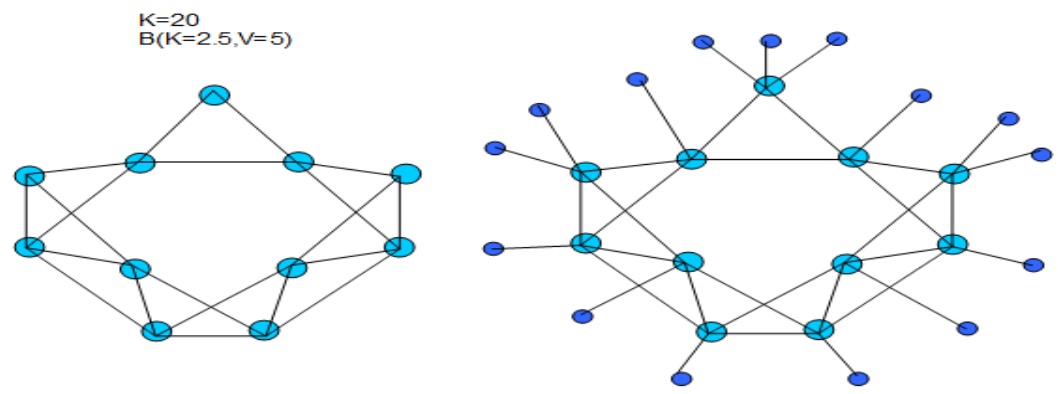

Figure 51. Isomeric graphical structure of $\mathrm{B}_{11} \mathrm{H}_{15}$

34. $\mathrm{B}_{10} \mathrm{H}_{16}: \mathrm{K}=10[2.5]-8=17, \mathrm{n}=10$

$\mathrm{K}(\mathrm{n})=17(10)$

$20-17=3$

$\mathrm{S}=4 \mathrm{n}+6$ (arachno)

$\mathrm{K}=2 \mathrm{n}-3$

$\mathrm{Kp}=\mathrm{C}^{-2} \mathrm{C}[\mathrm{M} 12]$

$\mathrm{K}^{*}=\mathrm{C}^{-2}+\mathrm{D}^{12}$

$\mathrm{y}=-2, \mathrm{z}=12$

$\mathrm{VE} 0=2 \mathrm{z}+2=2[12]+2=26$

$\mathrm{VE}=\mathrm{VE} 0+2 \mathrm{n}=26+2[10]=46$

$\mathrm{VE}=2 \mathrm{y}+4 \mathrm{z}+2=2[-2]+4[12]+2=-4+48+2=50-4=46$

$\mathrm{VF}=10[3]+16=30+16=46$

$$
\mathrm{K}=17
$$

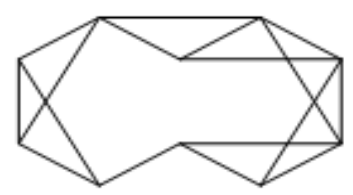

$B(K=2.5, V=5)$

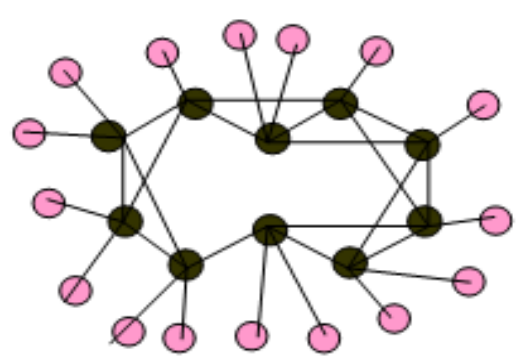

Figure 52. Isomeric graphical structure of $\mathrm{B}_{10} \mathrm{H}_{16}$ 
35. $\mathrm{B}_{9} \mathrm{H}_{17}: \mathrm{K}=9[2.5]-8.5=14, \mathrm{n}=9$

$\mathrm{K}(\mathrm{n})=14(9)$

$18-14=4$

$\mathrm{S}=4 \mathrm{n}+8$ (arachno)

$\mathrm{K}=2 \mathrm{n}-4$

$\mathrm{Kp}=\mathrm{C}^{-3} \mathrm{C}[\mathrm{M} 12]$

$\mathrm{K}^{*}=\mathrm{C}^{-3}+\mathrm{D}^{12}$

$\mathrm{y}=-3, \mathrm{z}=12$

$\mathrm{VE} 0=2 \mathrm{z}+2=2[12]+2=26$

$\mathrm{VE}=\mathrm{VE} 0+2 \mathrm{n}=26+2[9]=26+18=44$

$\mathrm{VE}=2 \mathrm{y}+4 \mathrm{z}+2=2[-3]+4[12]+2=-6+48+2=50-6=44$

$\mathrm{VF}=9[3]+17=27+17=44$
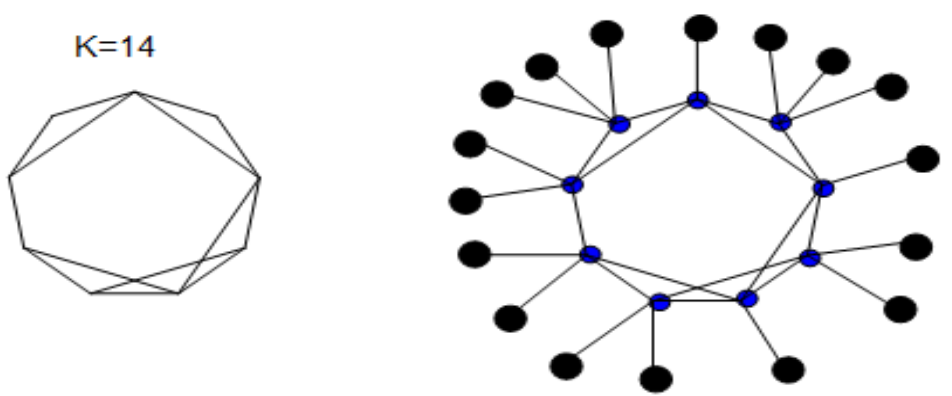

Figure 53. Isomeric graphical structure of $\mathrm{B}_{13} \mathrm{H}_{13}{ }^{2-}$

36. $\mathrm{B}_{8} \mathrm{H}_{18}: \mathrm{K}=8[2.5]-9=11, \mathrm{n}=8$

$\mathrm{K}(\mathrm{n})=11(8)$

$16-11=5$

$\mathrm{S}=4 \mathrm{n}+10$ (klapo)

$\mathrm{K}=2 \mathrm{n}-5$

$\mathrm{Kp}=\mathrm{C}^{-4} \mathrm{C}[\mathrm{M} 12]$

$\mathrm{K}^{*}=\mathrm{C}^{-4}+\mathrm{D}^{12}$

$\mathrm{y}=-4, \mathrm{z}=12$

$\mathrm{VE}=2 \mathrm{y}+4 \mathrm{z}+2=2[-4]+4[12]+2=-8+48+2=40+2=42$

$\mathrm{VE}=8 \mathrm{n}-2 \mathrm{~K}=8[8]-2[11]=64-22=42$

$\mathrm{VF}=8[3]+18=24+18=42$

$$
\begin{aligned}
& \mathrm{K}=11 \\
& \mathrm{~B}(\mathrm{~K}=2.5, \mathrm{v}=5)
\end{aligned}
$$
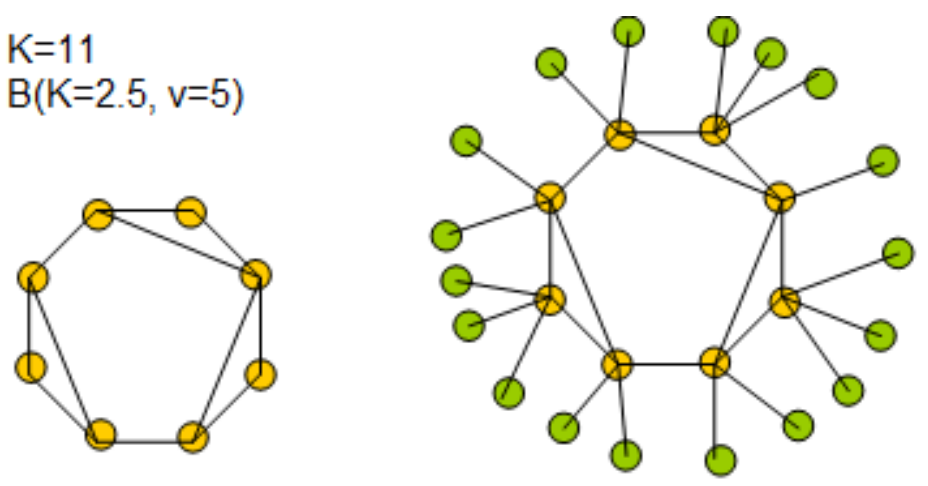

Figure 54. Isomeric graphical structure of $\mathrm{B}_{8} \mathrm{H}_{18}$ 


\section{$D^{13}$ SERIES}

37. $\mathrm{B}_{13} \mathrm{H}_{13}{ }^{2-}: \mathrm{K}=13[2.5]-6.5-1=25, \mathrm{n}=13$

$\mathrm{K}(\mathrm{n})=25(13)$

$26-25=1$

$\mathrm{S}=4 \mathrm{n}+2$ (closo)

$\mathrm{K}=2 \mathrm{n}-1$

$\mathrm{Kp}=\mathrm{C}^{0} \mathrm{C}[\mathrm{M} 13]$

$\mathrm{K}^{*}=\mathrm{C}^{0}+\mathrm{D}^{13}$

$\mathrm{y}=0, \mathrm{z}=13$

$\mathrm{VE} 0=2 \mathrm{z}+2=2[13]+2=28$

$\mathrm{VE}=\mathrm{VE} 0+2 \mathrm{n}=28+2[13]=28+26=54$

$\mathrm{VE}=8 \mathrm{n}-2 \mathrm{~K}=8[13]-2[25]=104-50=54$

$\mathrm{VE}=4 \mathrm{n}+2=4[13]+2=54$
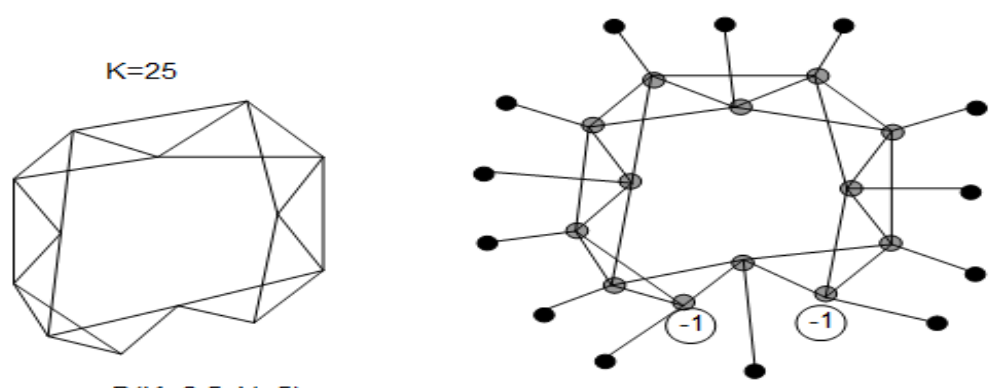

$\mathrm{B}(\mathrm{K}=2.5, \mathrm{~V}=5)$

Figure 55. Isomeric graphical structure of $\mathrm{B}_{8} \mathrm{H}_{18}$

38. $\mathrm{B}_{12} \mathrm{H}_{16}: \mathrm{K}=12[2.5]-8=22, \mathrm{n}=12$

$\mathrm{K}(\mathrm{n})=22(12)$

$24-22=2$

$\mathrm{S}=4 \mathrm{n}+4$ (nido)

$\mathrm{K}=2 \mathrm{n}-2$

$\mathrm{Kp}=\mathrm{C}-1 \mathrm{C}[\mathrm{M} 13]$

$\mathrm{K}^{*}=\mathrm{C}^{-1}+\mathrm{D}^{13}$

$\mathrm{y}=-1, \mathrm{z}=13$

$\mathrm{VE} 0=2 \mathrm{z}+2=2[13]+2=28$

$\mathrm{VE}=\mathrm{VE} 0+2 \mathrm{n}=28+2[12]=28+24=52$

$\mathrm{VE}=8 \mathrm{n}-2 \mathrm{~K}=8[12]-2[22]=96-44=52$

$\mathrm{VE}=4 \mathrm{n}+4=4[12]+4=52$

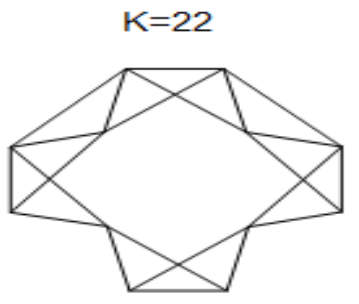

$B(K=2.5, V=55)$

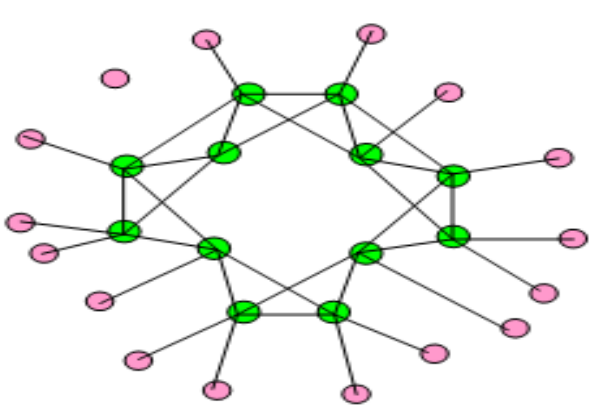

Figure 56. Isomeric graphical structure of $\mathrm{B}_{12} \mathrm{H}_{16}$ 


\section{$D^{14}$ SERIES}

39. $\mathrm{B}_{14} \mathrm{H}_{14}{ }^{2-}: \mathrm{K}=14[2.5]-7-1=27, \mathrm{n}=14$

$\mathrm{K}(\mathrm{n})=27(14)$

28-27=1

$\mathrm{S}=4 \mathrm{n}+2$ (closo)

$\mathrm{K}=2 \mathrm{n}-1$

$\mathrm{Kp}=\mathrm{C}^{0} \mathrm{C}[\mathrm{M} 14]$

$\mathrm{K}^{*}=\mathrm{C}^{0}+\mathrm{D}^{14}$

$\mathrm{y}=0, \mathrm{z}=14$

$\mathrm{VE} 0=2 \mathrm{z}+2=2[14]+2=30$

$\mathrm{VE}=\mathrm{VE} 0+2 \mathrm{n}=30+2[14]=30+28=58$

$\mathrm{VE}=8 \mathrm{n}-2 \mathrm{~K}=8[14]-2[27]=112-54=58$

$\mathrm{S}=4 \mathrm{n}+2=4[14]+2=58$

40. $\mathrm{B}_{13} \mathrm{H}_{17} \mathrm{~K}=13[2.5]-8.5=24, \mathrm{n}=13$

$\mathrm{K}(\mathrm{n})=24(13)$

26-24=2

$\mathrm{S}=4 \mathrm{n}+4$ (nido)

$\mathrm{K}=2 \mathrm{n}-2$

$\mathrm{Kp}=\mathrm{C}^{-1} \mathrm{C}[\mathrm{M} 14]$

$\mathrm{K}^{*}=\mathrm{C}^{-1}+\mathrm{D}^{14}$

$\mathrm{y}=-1, \mathrm{z}=14$

$\mathrm{VE} 0=2 \mathrm{z}+2=2[14]+2=30$

$\mathrm{VE}=\mathrm{VE} 0+2 \mathrm{n}=30+2[13]=56$

$\mathrm{VE}=8 \mathrm{n}-2 \mathrm{~K}=8[13]-2[24]=56$

$\mathrm{VE}=4 \mathrm{n}+4=4[13]+4=56$
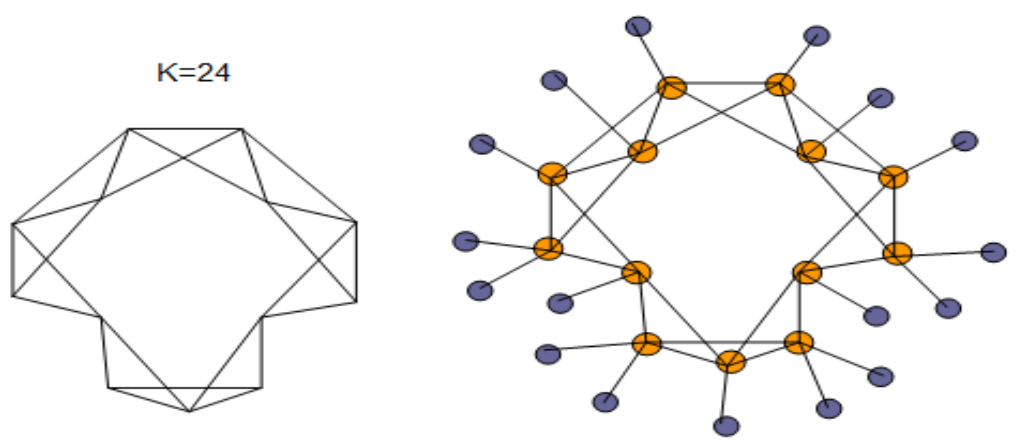

Figure 57. Isomeric graphical structure of $\mathrm{B}_{13} \mathrm{H}_{17}$

\section{$D^{15}$ SERIES}

41. $\mathrm{B}_{15} \mathrm{H}_{15}{ }^{2-}: \mathrm{K}=15[2.5]-7.5-1=29, \mathrm{n}=15$

$\mathrm{K}(\mathrm{n})=29(15)$

$30-29=1$

$\mathrm{S}=4 \mathrm{n}+2$ (closo)

$\mathrm{K}=2 \mathrm{n}-1$

$\mathrm{Kp}=\mathrm{C}^{0} \mathrm{C}[\mathrm{M} 15]$

$\mathrm{K}^{*}=\mathrm{C}^{0}+\mathrm{D}^{15}$ 
$\mathrm{y}=0, \mathrm{z}=15$

$\mathrm{VE} 0=2 \mathrm{z}+2=2[15]+2=32$

$\mathrm{VE}=\mathrm{VE} 0+2 \mathrm{n}=32+2[15]=32+30=62$

$\mathrm{VE}=8 \mathrm{n}-2 \mathrm{~K}=8[15]-2[29]=120-58=62$

$\mathrm{VE}=4 \mathrm{n}+2=4[15]+2=62$
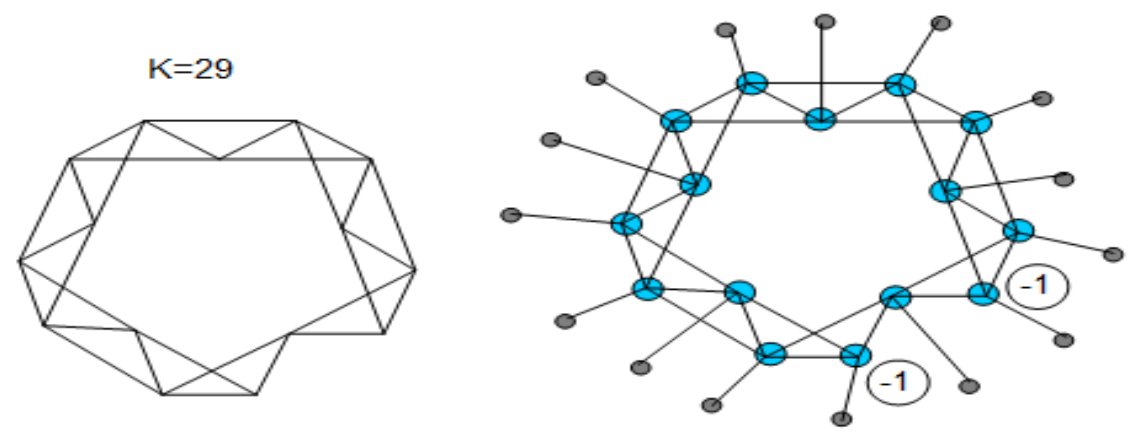

Figure 58. Isomeric graphical structure of $\mathrm{B}_{15} \mathrm{H}_{15}{ }^{2-}$

42. $\mathrm{B}_{14} \mathrm{H}_{18}: \mathrm{K}=14[2.5]-9=26, \mathrm{n}=14$

$\mathrm{K}(\mathrm{n})=26(14)$

$28-26=2$

$\mathrm{S}=4 \mathrm{n}+4$ (nido)

$\mathrm{K}=2 \mathrm{n}-2$

$\mathrm{Kp}=\mathrm{C}^{-1} \mathrm{C}[\mathrm{M} 15]$

$\mathrm{K}^{*}=\mathrm{C}^{-1}+\mathrm{D}^{15}$

$\mathrm{y}=-1, \mathrm{z}=15$

$\mathrm{VE} 0=2 \mathrm{z}+2=2[15]+2=32$

$\mathrm{VE}=\mathrm{VE} 0+2 \mathrm{n}=32+2[14]=32+28=60$

$\mathrm{VE}=8 \mathrm{n}-2 \mathrm{~K}=8[14]-2[26]=112-52=60$

$\mathrm{VE}=4 \mathrm{n}+4=4[14]+4=60$
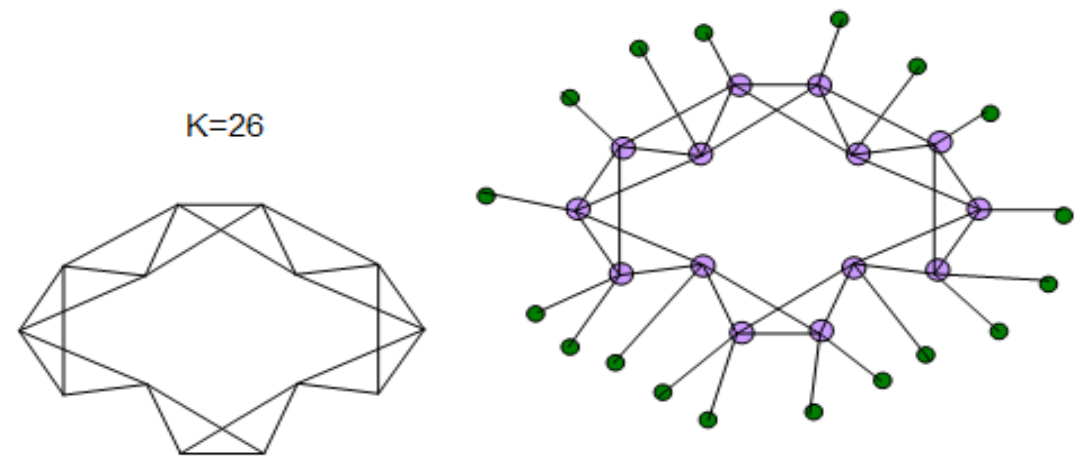

Figure 59. Isomeric graphical structure of $\mathrm{B}_{14} \mathrm{H}_{18}$

43. $\mathrm{B}_{13} \mathrm{H}_{19}: \mathrm{K}=13[2.5]-9.5=23, \mathrm{n}=13$

$\mathrm{K}(\mathrm{n})=23(13)$

$26-23=3$

$S=4 n+6$ (arachno)

$\mathrm{K}=2 \mathrm{n}-3$

$\mathrm{Kp}=\mathrm{C}^{-2} \mathrm{C}[\mathrm{M} 15]$

$\mathrm{K}^{*}=\mathrm{C}^{-2}+\mathrm{D}^{15}$ 
$\mathrm{y}=-2, \mathrm{z}=15$

$\mathrm{VE} 0=2 \mathrm{z}+2=2[13]+2=28$

$\mathrm{VE}=\mathrm{VE} 0+2 \mathrm{n}=28+2[13]=28+26=54$

44. $\mathrm{B}_{12} \mathrm{H}_{20}: \mathrm{K}=12[2.5]-10=20, \mathrm{n}=12$

$\mathrm{K}(\mathrm{n})=20(12)$

$24-20=4$

$\mathrm{S}=4 \mathrm{n}+8$ (hypho)

$\mathrm{K}=2 \mathrm{n}-4$

$\mathrm{Kp}=\mathrm{C}^{-3} \mathrm{C}[\mathrm{M} 15]$

$\mathrm{K}^{*}=\mathrm{C}^{-3}+\mathrm{D}^{15}$

$\mathrm{y}=-3, \mathrm{z}=15$

$\mathrm{VE} 0=2 \mathrm{z}+2=2[15]+2=32$

$\mathrm{VE}=\mathrm{VE} 0+2 \mathrm{n}=32+2[12]=32+24=56$

$\mathrm{VF}=12[3]+20=36+20=56$

$$
K=20
$$

$\mathrm{B}(\mathrm{K}=2.5, \mathrm{~V}=5)$
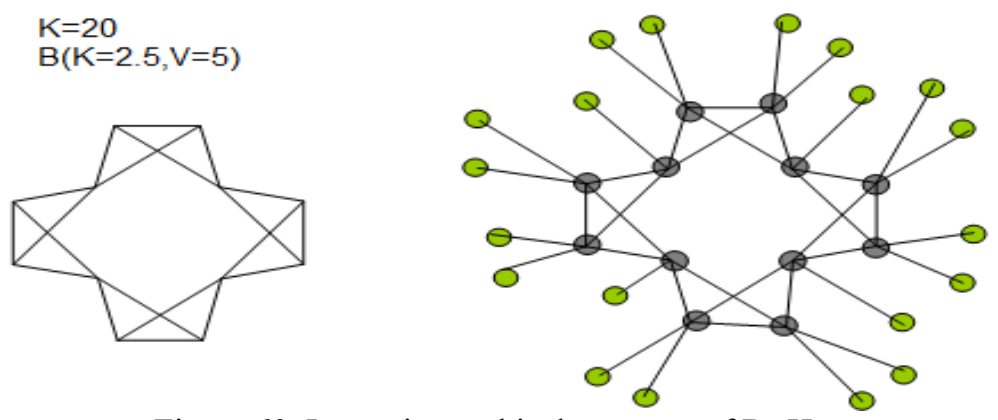

Figure 60. Isomeric graphical structure of $\mathrm{B}_{12} \mathrm{H}_{20}$

45. $\mathrm{B}_{11} \mathrm{H}_{21}: \mathrm{K}=11[2.5]-10.5=17, \mathrm{n}=11$

$\mathrm{K}(\mathrm{n})=17(11)$

$22-17=5$

$\mathrm{S}=4 \mathrm{n}+10$ (klapo)

$\mathrm{K}=2 \mathrm{n}-5$

$\mathrm{Kp}=\mathrm{C}^{-4} \mathrm{C}[\mathrm{M} 15]$

$\mathrm{K}^{*}=\mathrm{C}^{-4}+\mathrm{D}^{15}$

$\mathrm{y}=-4, \mathrm{z}=15$

$\mathrm{VE} 0=2 \mathrm{z}+2=2[15]+2=32$

$\mathrm{VE}=\mathrm{VE} 0+2 \mathrm{n}=32+2[11]=32+22=54$

$\mathrm{VF}=11[3]+21=33+21=54$
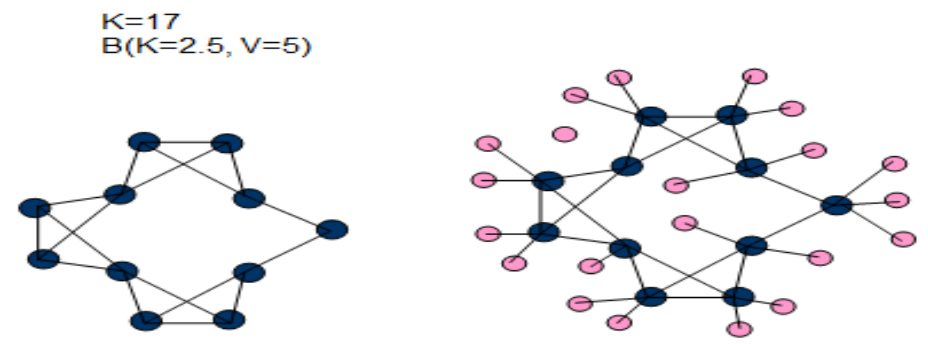

Figure 61. Isomeric graphical structure of $\mathrm{B}_{11} \mathrm{H}_{21}$ 
46. $\mathrm{B}_{10} \mathrm{H}_{22}: \mathrm{K}=10[2.5]-11=14, \mathrm{n}=10$

$\mathrm{K}(\mathrm{n})=14(10)$

20-14=6

$\mathrm{S}=4 \mathrm{n}+12$ (klapo)

$\mathrm{K}=2 \mathrm{n}-6$

$\mathrm{Kp}=\mathrm{C}^{-5} \mathrm{C}[\mathrm{M} 15]$

$\mathrm{K}^{*}=\mathrm{C}^{-5}+\mathrm{D}^{15}$

$\mathrm{y}=-5, \mathrm{z}=15$

$\mathrm{VE} 0=2 \mathrm{z}+2=2[15]+2=32$

$\mathrm{VE}=\mathrm{VE} 0+2 \mathrm{n}=32+2[10]=32+20=52$

$\mathrm{VF}=10[3]+22=30+22=52$

\section{$\mathrm{K}=14$}

$B(K=2.5, V=5)$
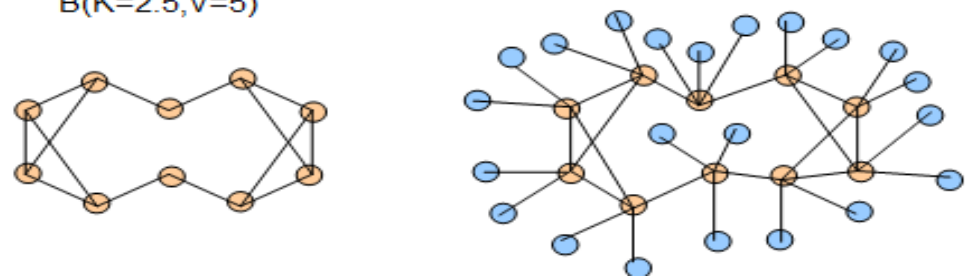

Figure 62. Isomeric graphical structure of $\mathrm{B}_{10} \mathrm{H}_{22}$

49. $\mathrm{B}_{23} \mathrm{H}_{19}: \mathrm{K}=23[2.5]-19[0.5]=48, \mathrm{n}=23$

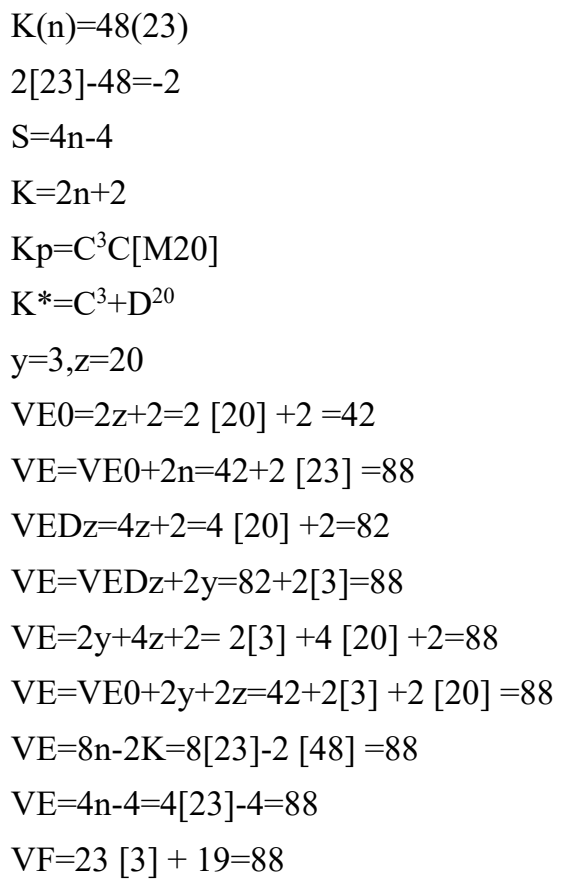




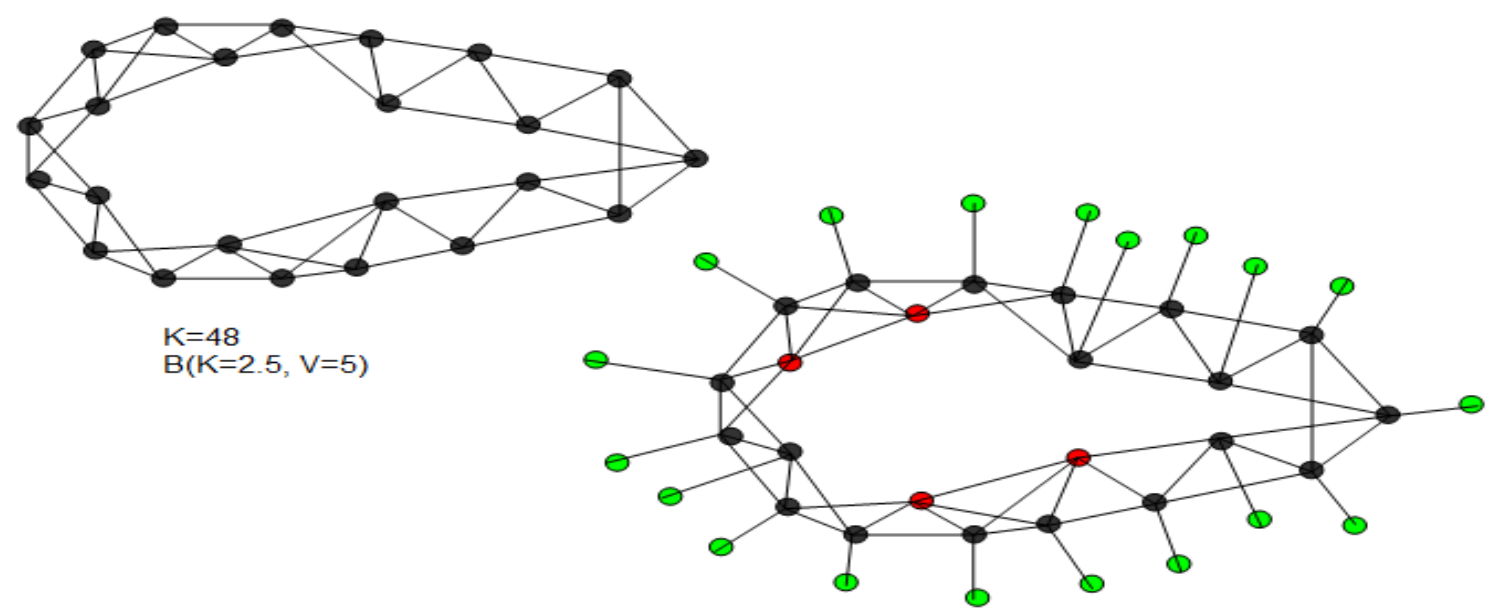

Figure 63. Isomeric graphical structure of $\mathrm{B}_{23} \mathrm{H}_{19}$
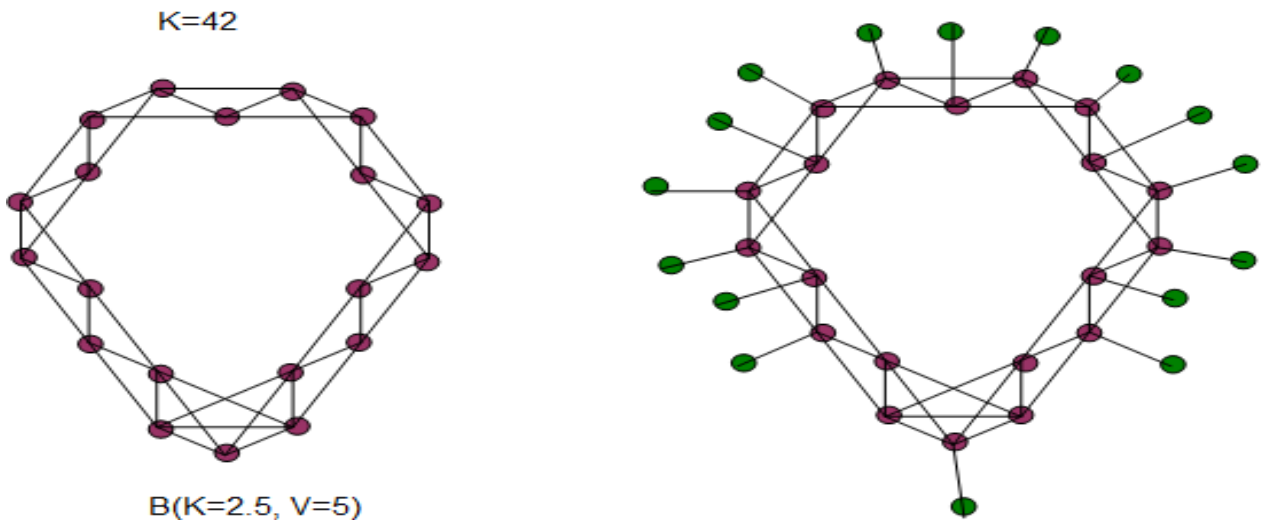

Figure 64. Isomeric graphical structure of $\mathrm{B}_{20} \mathrm{H}_{16}$

$K(n)=34(18)$

$34=18+16$
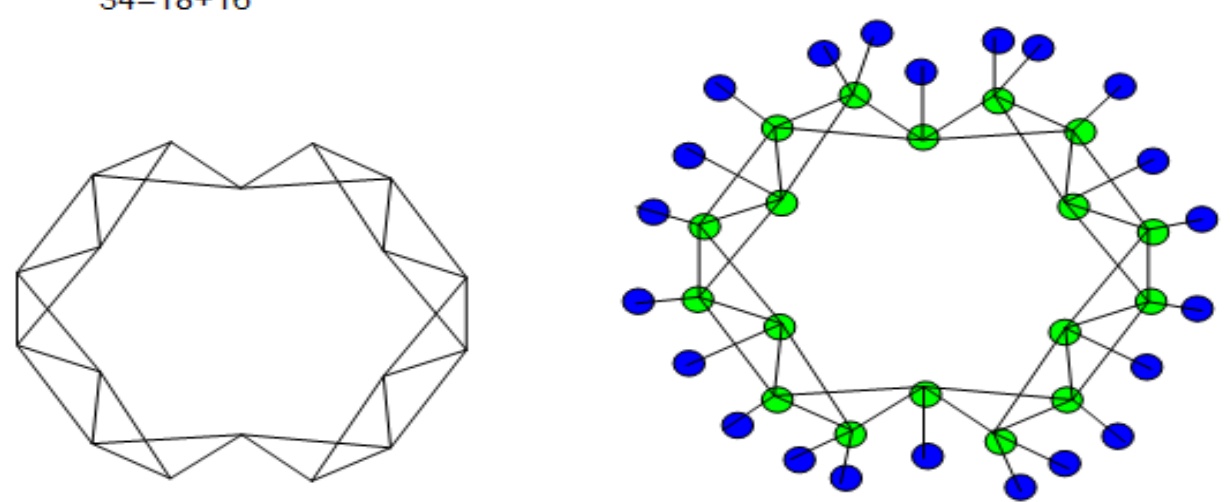

Figure 65. Isomeric graphical structure of $\mathrm{B}_{18} \mathrm{H}_{22}$ 
Table 1. Categorization of Borane Clusters into Clan Series

\begin{tabular}{|c|c|c|c|c|c|c|c|c|c|c|}
\hline & Cluster & & $\mathrm{K}(\mathrm{n})$ & $S=4 n+q$ & $\begin{array}{l}\text { Local } \\
\text { name }\end{array}$ & $\begin{array}{c}\mathrm{K}^{*}= \\
\mathrm{C}^{\mathrm{y}}+\mathrm{D}^{\mathrm{z}}\end{array}$ & $\mathrm{y}, \mathrm{z}$ & $\mathrm{VE} 0=2 \mathrm{z}+2$ & $V E=2 y+4 z+2$ & VF \\
\hline 1 & $\mathrm{BH}_{3}$ & & 1(1) & $4 n+2$ & closo & $\mathrm{C}^{0}+\mathrm{D}^{1}$ & 0,1 & $2[1]+2=4$ & $\begin{array}{c}2[0]+4[1] \\
+2=6\end{array}$ & $1[3]+3=6$ \\
\hline 2 & $\mathrm{BH}_{4}^{-}$ & & $0(1)$ & $4 n+4$ & nido & $\mathrm{C}^{-1}+\mathrm{D}^{2}$ & $-1,2$ & $2[2]+2=6$ & $\begin{array}{c}2[-1]+4[2] \\
+2=8\end{array}$ & $1[3]+4+1=8$ \\
\hline 3 & $\mathrm{~B}_{2} \mathrm{H}_{6}$ & & $2(2)$ & $4 n+4$ & nido & $\mathrm{C}^{-1}+\mathrm{D}^{3}$ & $-1,3$ & $2[3]+2=8$ & $\begin{array}{l}2[-1]+4[3] \\
\quad+2=12\end{array}$ & $2[3]+6=12$ \\
\hline 4 & $\mathrm{~B}_{2} \mathrm{H}_{8}$ & & $1(2)$ & $4 n+6$ & arachno & $\mathrm{C}^{-2}+\mathrm{D}^{4}$ & $-2,4$ & $2[4]+2=10$ & $\begin{array}{l}2[-2]+4[4] \\
\quad+2=14\end{array}$ & $2[3]+8=14$ \\
\hline 5 & $\mathrm{~B}_{3} \mathrm{H}_{9}$ & & $3(3)$ & $4 n+6$ & arachno & $\mathrm{C}^{-2}+\mathrm{D}^{5}$ & $-2,5$ & $2[5]+2=12$ & $\begin{array}{l}2[-2]+4[5] \\
\quad+2=18\end{array}$ & $3[3]+9=18$ \\
\hline 6 & $\mathrm{~B}_{4} \mathrm{H}_{8}$ & & $6(4)$ & $4 n+4$ & nido & $\mathrm{C}^{-1}+\mathrm{D}^{5}$ & $-1,5$ & $2[5]+2=12$ & $\begin{array}{l}2[-1]+4[5] \\
\quad+2=20\end{array}$ & $4[3]+8=20$ \\
\hline 7 & $\mathrm{~B}_{5} \mathrm{H}_{5}{ }^{2-}$ & & $9(5)$ & $4 n+2$ & closo & $\mathrm{C}^{0}+\mathrm{D}^{5}$ & 0,5 & $2[5]+2=12$ & $\begin{array}{l}2[0]+4[5] \\
\quad+2=22\end{array}$ & $\begin{array}{l}5[3] \\
+5+2=22\end{array}$ \\
\hline 8 & $\mathrm{~B}_{4} \mathrm{H}_{8}$ & & $6(4)$ & $4 n+4$ & nido & $\mathrm{C}^{-1}+\mathrm{D}^{5}$ & $-1,5$ & $2[5]+2=12$ & $\begin{array}{l}2[-1]+4[5] \\
\quad+2=20\end{array}$ & $4[3]+8=20$ \\
\hline 9 & $\mathrm{~B}_{3} \mathrm{H}_{9}$ & & $3(3)$ & $4 n+6$ & arachno & $\mathrm{C}^{-2}+\mathrm{D}^{5}$ & $-2,5$ & $2[5]+2=12$ & $\begin{array}{l}2[-2]+4[5] \\
\quad+2=18\end{array}$ & $3[3]+9=18$ \\
\hline 10 & $\mathrm{~B}_{6} \mathrm{H}_{6}{ }^{2-}$ & & 11(6) & $4 n+2$ & closo & $\mathrm{C}^{0}+\mathrm{D}^{6}$ & 0,6 & $2[6]+2=14$ & $\begin{array}{l}2[0]+4[6] \\
\quad+2=26\end{array}$ & $\begin{array}{l}6[3] \\
+6+2=26\end{array}$ \\
\hline 11 & $\mathrm{~B}_{5} \mathrm{H}_{9}$ & & $8(5)$ & $4 n+4$ & nido & $\mathrm{C}^{-1}+\mathrm{D}^{6}$ & $-1,6$ & $2[6]+2=14$ & $\begin{array}{l}2[-1]+4[6] \\
+2=24\end{array}$ & $5[3]+9=24$ \\
\hline 12 & $\mathrm{~B}_{4} \mathrm{H}_{10}$ & & $5(4)$ & $4 n+6$ & arachno & $\mathrm{C}^{-2}+\mathrm{D}^{6}$ & $-2,6$ & $2[6]+2=14$ & $\begin{array}{l}2[-2]+4[6] \\
+2=22\end{array}$ & $4[3]+10=22$ \\
\hline 13 & $\mathrm{~B}_{6} \mathrm{H}_{14}$ & & $8(6)$ & $4 n+8$ & hypho & $\mathrm{C}^{-3}+\mathrm{D}^{9}$ & $-3,9$ & $2[9]+2=20$ & $\begin{array}{l}2[-3]+4[9] \\
\quad+2=32\end{array}$ & $6[3]+14=32$ \\
\hline 14 & $\mathrm{~B}_{7} \mathrm{H}_{7}{ }^{2-}$ & & $13(7)$ & $4 n+2$ & closo & $\mathrm{C}^{0}+\mathrm{D}^{7}$ & 0,7 & $2[7]+2=16$ & $\begin{array}{c}2[0]+4[7] \\
+2=30\end{array}$ & $\begin{array}{l}7[3] \\
+7+2=30\end{array}$ \\
\hline 15 & $\mathrm{~B}_{6} \mathrm{H}_{10}$ & & $10(6)$ & $4 n+4$ & nido & $\mathrm{C}^{-1}+\mathrm{D}^{7}$ & $-1,7$ & $2[7]+2=16$ & $\begin{array}{c}2[-1]+4[7] \\
+2=28\end{array}$ & $6[3]+10=28$ \\
\hline 16 & $\mathrm{~B}_{5} \mathrm{H}_{11}$ & & $7(5)$ & $4 n+6$ & arachno & $\mathrm{C}^{-2}+\mathrm{D}^{7}$ & $-2,7$ & $2[7]+2=16$ & $\begin{array}{l}2[-2]+4[7] \\
\quad+2=26\end{array}$ & $5[3]+11=26$ \\
\hline 17 & $\mathrm{~B}_{6} \mathrm{H}_{12}$ & $9(6)$ & $4 n+6$ & arachno & $\mathrm{C}^{-2}+\mathrm{D}^{8}$ & $-2,8$ & $\begin{array}{c}2[8] \\
+2=18\end{array}$ & $\begin{array}{l}2[-2]+4[8] \\
\quad+2=30\end{array}$ & $6[3]+12=30$ & \\
\hline 18 & $\mathrm{~B}_{7} \mathrm{H}_{11}$ & $12(7)$ & $4 n+4$ & nido & $\mathrm{C}^{-1}+\mathrm{D}^{8}$ & $-1,8$ & $\begin{array}{c}2[8] \\
+2=18\end{array}$ & $\begin{array}{l}2[-1]+4[8] \\
\quad+2=32\end{array}$ & $7[3]+11=32$ & \\
\hline 19 & $\mathrm{~B}_{8} \mathrm{H}_{8}{ }^{2-}$ & $15(8)$ & $4 n+2$ & closo & $\mathrm{C}^{0}+\mathrm{D}^{8}$ & 0,8 & $\begin{array}{c}2[8] \\
+2=18\end{array}$ & $\begin{array}{c}2[0]+4[8] \\
+2=34\end{array}$ & $8[3]+8+2=34$ & \\
\hline 20 & $\mathrm{~B}_{7} \mathrm{H}_{11}$ & $12(7)$ & $4 n+4$ & nido & $\mathrm{C}^{-1}+\mathrm{D}^{8}$ & $-1,8$ & $\begin{array}{c}2[8] \\
+2=18 \\
\end{array}$ & $\begin{array}{l}2[-1]+4[8] \\
+2=32\end{array}$ & $7[3]+11=32$ & \\
\hline 21 & $\mathrm{~B}_{6} \mathrm{H}_{12}$ & $9(6)$ & $4 n+6$ & arachno & $\mathrm{C}^{-2}+\mathrm{D}^{8}$ & $-2,8$ & $\begin{array}{c}2[8] \\
+2=18\end{array}$ & $\begin{array}{l}2[-2]+4[8] \\
+2=30\end{array}$ & $6[3]+12=30$ & \\
\hline 22 & $\mathrm{~B}_{9} \mathrm{H}_{9}{ }^{2-}$ & 17(9) & $4 n+2$ & closo & $\mathrm{C}^{0}+\mathrm{D}^{9}$ & 0,9 & $\begin{array}{c}2[9] \\
+2=20\end{array}$ & $\begin{array}{l}2[0]+4[9] \\
+2=38\end{array}$ & $9[3]+9+2=38$ & \\
\hline 23 & $\mathrm{~B}_{8} \mathrm{H}_{12}$ & $14(8)$ & $4 n+4$ & nido & $\mathrm{C}^{-1}+\mathrm{D}^{9}$ & $-1,9$ & $\begin{array}{c}2[9] \\
+2=20\end{array}$ & $\begin{array}{c}2[-1]+4[9] \\
+2=36\end{array}$ & $8[3]+12=36$ & \\
\hline 24 & $\mathrm{~B}_{7} \mathrm{H}_{13}$ & $11(7)$ & $4 n+6$ & arachno & $\mathrm{C}^{-2}+\mathrm{D}^{9}$ & $-2,9$ & $\begin{array}{c}2[9] \\
+2=20\end{array}$ & $\begin{array}{c}2[-2]+4[9] \\
+2=34\end{array}$ & $7[3]+13=34$ & \\
\hline 25 & $\mathrm{~B}_{6} \mathrm{H}_{14}$ & $8(6)$ & $4 n+8$ & hypho & $\mathrm{C}^{-3}+\mathrm{D}^{9}$ & $-3,9$ & $\begin{array}{c}2[9] \\
+2=20\end{array}$ & $\begin{array}{l}2[-3]+4[9] \\
\quad+2=32\end{array}$ & $6[3]+14=32$ & \\
\hline 26 & $\mathrm{~B}_{9} \mathrm{H}_{13}$ & $16(9)$ & $4 n+4$ & nido & $\mathrm{C}^{-1}+\mathrm{D}^{10}$ & $-1,10$ & $\begin{array}{r}2[10] \\
+2=22\end{array}$ & $\begin{array}{c}2[-1]+4[10] \\
+2=40\end{array}$ & $9[3]+13=40$ & \\
\hline 27 & $\mathrm{~B}_{8} \mathrm{H}_{14}$ & $13(8)$ & $4 n+6$ & arachno & $\mathrm{C}^{-2}+\mathrm{D}^{10}$ & $-2,10$ & $\begin{array}{l}2[10] \\
+2=22\end{array}$ & $\begin{array}{c}2[-2]+4[10] \\
+2=38\end{array}$ & $8[3]+14=38$ & \\
\hline 28 & $\mathrm{~B}_{10} \mathrm{H}_{10}{ }^{2-}$ & 19(10) & $4 n+2$ & closo & $\mathrm{C}^{0}+\mathrm{D}^{10}$ & 0,10 & $\begin{array}{r}2[10] \\
+2=22\end{array}$ & $\begin{array}{c}2[0]+4[10] \\
+2=42\end{array}$ & $\begin{array}{c}10[3] \\
+10+2=42\end{array}$ & \\
\hline 29 & $\mathrm{~B}_{9} \mathrm{H}_{13}$ & $16(9)$ & $4 n+4$ & nido & $\mathrm{C}^{-1}+\mathrm{D}^{10}$ & $-1,10$ & $\begin{array}{r}2[10] \\
+2=22\end{array}$ & $\begin{array}{c}2[-1]+4[10] \\
+2=40\end{array}$ & $9[3]+13=40$ & \\
\hline 30 & $\mathrm{~B}_{8} \mathrm{H}_{14}$ & $13(8)$ & $4 n+6$ & arachno & $\mathrm{C}^{-2}+\mathrm{D}^{10}$ & $-2,10$ & $\begin{array}{r}2[10] \\
+2=22\end{array}$ & $\begin{array}{c}2[-2]+4[10] \\
+2=38\end{array}$ & $8[3]+14=38$ & \\
\hline 31 & $\mathrm{~B}_{7} \mathrm{H}_{15}$ & $10(7)$ & $4 n+8$ & hypho & $\mathrm{C}^{-3}+\mathrm{D}^{10}$ & $-3,10$ & $\begin{array}{r}2[10] \\
+2=22\end{array}$ & $\begin{array}{c}2[-3]+4[10] \\
+2=36\end{array}$ & $7[3]+15=36$ & \\
\hline 32 & $\mathrm{~B}_{11} \mathrm{H}_{11}{ }^{2-}$ & $21(11)$ & $4 n+2$ & closo & $\mathrm{C}^{0}+\mathrm{D}^{11}$ & 0,11 & $\begin{array}{r}2[11] \\
+2=24\end{array}$ & $\begin{array}{c}2[0]+4[11] \\
+2=46\end{array}$ & $\begin{array}{c}11[3] \\
+11+2=46\end{array}$ & \\
\hline 33 & $\mathrm{~B}_{10} \mathrm{H}_{14}$ & $18(10)$ & $4 n+4$ & nido & $\mathrm{C}^{-1}+\mathrm{D}^{11}$ & $-1,11$ & $\begin{array}{r}2[11] \\
+2=24\end{array}$ & $\begin{array}{c}2[-1]+4[11] \\
+2=44\end{array}$ & $10[3]+14=44$ & \\
\hline 34 & $\mathrm{~B}_{9} \mathrm{H}_{15}$ & $15(9)$ & $4 n+6$ & arachno & $\mathrm{C}^{-2}+\mathrm{D}^{11}$ & $-2,11$ & $\begin{array}{r}2[11] \\
+2=24\end{array}$ & $\begin{array}{c}2[-2]+4[11] \\
+2=42\end{array}$ & $9[3]+15=42$ & \\
\hline 35 & $\mathrm{~B}_{8} \mathrm{H}_{16}$ & $12(8)$ & $4 n+8$ & hypho & $\mathrm{C}^{-3}+\mathrm{D}^{11}$ & $-3,11$ & $2[11]$ & $2[-3]+4[11]$ & $8[3]+16=40$ & \\
\hline
\end{tabular}




\begin{tabular}{|c|c|c|c|c|c|c|c|c|c|}
\hline & & & & & & & $+2=24$ & $+2=40$ & \\
\hline 36 & $\mathrm{~B}_{9} \mathrm{H}_{15}$ & $15(9)$ & $4 n+6$ & arachno & $\mathrm{C}^{-2}+\mathrm{D}^{11}$ & $-2,11$ & $\begin{array}{r}2[11] \\
+2=24 \\
\end{array}$ & $\begin{array}{c}2[-2]+4[11] \\
+2=42\end{array}$ & $9[3]+15=42$ \\
\hline 37 & $\mathrm{~B}_{10} \mathrm{H}_{14}$ & $18(10)$ & $4 n+4$ & nido & $\mathrm{C}^{-1}+\mathrm{D}^{11}$ & $-1,11$ & $\begin{array}{r}2[11] \\
+2=24\end{array}$ & $\begin{array}{c}2[-1]+4[11] \\
+2=44\end{array}$ & $10[3]+14=44$ \\
\hline 38 & $\mathrm{~B}_{12} \mathrm{H}_{12}{ }^{2-}$ & $23(12)$ & $4 n+2$ & closo & $\mathrm{C}^{0}+\mathrm{D}^{12}$ & 0,12 & $\begin{array}{r}2[12] \\
+2=26\end{array}$ & $\begin{array}{c}2[0]+4[12] \\
+2=50\end{array}$ & $\begin{array}{c}12[3] \\
+12+2=50\end{array}$ \\
\hline 39 & $\mathrm{~B}_{11} \mathrm{H}_{15}$ & $20(11)$ & $4 n+4$ & nido & $\mathrm{C}^{-1}+\mathrm{D}^{12}$ & $-1,12$ & $\begin{array}{r}2[12] \\
+2=26\end{array}$ & $\begin{array}{c}2[-1]+4[12] \\
+2=48\end{array}$ & $11[3]+15=48$ \\
\hline 40 & $\mathrm{~B}_{10} \mathrm{H}_{16}$ & $17(10)$ & $4 n+6$ & arachno & $\mathrm{C}^{-2}+\mathrm{D}^{12}$ & $-2,12$ & $\begin{array}{r}2[12] \\
+2=26\end{array}$ & $\begin{array}{c}2[-2]+4[12] \\
+2=46\end{array}$ & $10[3]+16=46$ \\
\hline 41 & $\mathrm{~B}_{9} \mathrm{H}_{17}$ & 14(9) & $4 n+8$ & hypho & $\mathrm{C}^{-3}+\mathrm{D}^{12}$ & $-3,12$ & $\begin{array}{r}2[12] \\
+2=26\end{array}$ & $\begin{array}{c}2[-3]+4[12] \\
+2=44\end{array}$ & $9[3]+17=44$ \\
\hline 42 & $\mathrm{~B}_{8} \mathrm{H}_{18}$ & $11(8)$ & $4 n+10$ & klapo & $\mathrm{C}^{-4}+\mathrm{D}^{12}$ & $-4,12$ & $\begin{array}{r}2[12] \\
+2=26\end{array}$ & $\begin{array}{c}2[-4]+4[12] \\
+2=42\end{array}$ & $8[3]+18=42$ \\
\hline 43 & $\mathrm{~B}_{13} \mathrm{H}_{13}{ }^{2-}$ & $25(13)$ & $4 n+2$ & closo & $\mathrm{C}^{0}+\mathrm{D}^{13}$ & 0,13 & $\begin{array}{r}2[13] \\
+2=28\end{array}$ & $\begin{array}{c}2[0]+4[13] \\
+2=54\end{array}$ & $\begin{array}{c}13[3] \\
+13+2=54\end{array}$ \\
\hline 44 & $\mathrm{~B}_{12} \mathrm{H}_{16}$ & $22(12)$ & $4 n+4$ & nido & $\mathrm{C}^{-1}+\mathrm{D}^{13}$ & $-1,13$ & $\begin{array}{r}2[13] \\
+2=28\end{array}$ & $\begin{array}{c}2[-1]+4[13] \\
+2=52\end{array}$ & $12[3]+16=52$ \\
\hline 45 & $\mathrm{~B}_{11} \mathrm{H}_{17}$ & 19(11) & $4 n+6$ & arachno & $\mathrm{C}^{-2}+\mathrm{D}^{13}$ & $-2,13$ & $\begin{array}{r}2[13] \\
+2=28\end{array}$ & $\begin{array}{c}2[-2]+4[13] \\
+2=50\end{array}$ & $11[3]+17=50$ \\
\hline 46 & $\mathrm{~B}_{10} \mathrm{H}_{18}$ & $16(10)$ & $4 n+8$ & hypho & $\mathrm{C}^{-3}+\mathrm{D}^{13}$ & $-3,13$ & $\begin{array}{r}2[13] \\
+2=28\end{array}$ & $\begin{array}{c}2[-3]+4[13] \\
+2=48\end{array}$ & $10[3]+18=48$ \\
\hline 47 & $\mathrm{~B}_{14} \mathrm{H}_{14}{ }^{2-}$ & $27(14)$ & $4 n+2$ & closo & $\mathrm{C}^{0}+\mathrm{D}^{14}$ & 0,14 & $\begin{array}{c}2[14] \\
+2=30\end{array}$ & $\begin{array}{c}2[0]+4[14] \\
+2=58\end{array}$ & $\begin{array}{c}14[3] \\
+14+2=58\end{array}$ \\
\hline 48 & $\mathrm{~B}_{13} \mathrm{H}_{17}$ & $24(13)$ & $4 n+4$ & nido & $\mathrm{C}^{-1}+\mathrm{D}^{14}$ & $-1,14$ & $\begin{array}{r}2[14] \\
+2=30 \\
\end{array}$ & $\begin{array}{c}2[-1]+4[14] \\
+2=56\end{array}$ & $13[3]+17=56$ \\
\hline 49 & $\mathrm{~B}_{12} \mathrm{H}_{18}$ & $21(12)$ & $4 n+6$ & arachno & $\mathrm{C}^{-2}+\mathrm{D}^{14}$ & $-2,14$ & $\begin{array}{r}2[14] \\
+2=30 \\
\end{array}$ & $\begin{array}{c}2[-2]+4[14] \\
+2=54\end{array}$ & $12[3]+18=54$ \\
\hline 50 & $\mathrm{~B}_{11} \mathrm{H}_{19}$ & $18(11)$ & $4 n+8$ & hypho & $\mathrm{C}^{-3}+\mathrm{D}^{14}$ & $-3,14$ & $\begin{array}{c}2[14] \\
+2=30\end{array}$ & $\begin{array}{c}2[-3]+4[14] \\
+2=52\end{array}$ & $11[3]+19=52$ \\
\hline 51 & $\mathrm{~B}_{15} \mathrm{H}_{15}{ }^{2-}$ & $29(15)$ & $4 n+2$ & closo & $\mathrm{C}^{0}+\mathrm{D}^{15}$ & 0,15 & $\begin{array}{r}2[15] \\
+2=32\end{array}$ & $\begin{array}{c}2[0]+4[15] \\
+2=62\end{array}$ & $\begin{array}{c}15[3] \\
+15+2=62\end{array}$ \\
\hline 52 & $\mathrm{~B}_{14} \mathrm{H}_{18}$ & $26(14)$ & $4 n+4$ & ndo & $\mathrm{C}^{-1}+\mathrm{D}^{15}$ & $-1,15$ & $\begin{array}{r}2[15] \\
+2=32\end{array}$ & $\begin{array}{c}2[-1]+4[15] \\
+2=60\end{array}$ & $14[3]+18=60$ \\
\hline 53 & $\mathrm{~B}_{13} \mathrm{H}_{19}$ & $23(13)$ & $4 n+6$ & arachno & $\mathrm{C}^{-2}+\mathrm{D}^{15}$ & $-2,15$ & $\begin{array}{r}2[15] \\
+2=32\end{array}$ & $\begin{array}{c}2[-2]+4[15] \\
+2=58\end{array}$ & $13[3]+19=58$ \\
\hline 54 & $\mathrm{~B}_{12} \mathrm{H}_{20}$ & $20(12)$ & $4 n+8$ & hypho & $\mathrm{C}^{-3}+\mathrm{D}^{15}$ & $-3,15$ & $\begin{array}{r}2[15] \\
+2=32\end{array}$ & $\begin{array}{c}2[-3]+4[15] \\
+2=56\end{array}$ & $12[3]+20=56$ \\
\hline 55 & $\mathrm{~B}_{11} \mathrm{H}_{21}$ & $17(11)$ & $4 n+10$ & klapo & $\mathrm{C}^{-4}+\mathrm{D}^{15}$ & $-4,15$ & $\begin{array}{r}2[15] \\
+2=32\end{array}$ & $\begin{array}{c}2[-4]+4[15] \\
+2=54\end{array}$ & $11[3]+21=54$ \\
\hline 56 & $\mathrm{~B}_{10} \mathrm{H}_{22}$ & $14(10)$ & $4 n+12$ & & $\mathrm{C}^{-5}+\mathrm{D}^{15}$ & $-5,15$ & $\begin{array}{r}2[15] \\
+2=32\end{array}$ & $\begin{array}{c}2[-5]+4[15] \\
+2=52\end{array}$ & $10[3]+22=52$ \\
\hline 57 & $\mathrm{~B}_{9} \mathrm{H}_{23}$ & 11(9) & $4 n+14$ & & $\mathrm{C}^{-6}+\mathrm{D}^{15}$ & $-6,15$ & $\begin{array}{r}2[15] \\
+2=32 \\
\end{array}$ & $\begin{array}{c}2[-5]+4[15] \\
+2=50\end{array}$ & $9[3]+23=50$ \\
\hline 58 & $\mathrm{~B}_{14} \mathrm{H}_{20}$ & $25(14)$ & $4 n+6$ & arachno & $\mathrm{C}^{-2}+\mathrm{D}^{16}$ & $-2,16$ & $\begin{array}{r}2[16] \\
+2=34\end{array}$ & $\begin{array}{c}2[-2]+4[16] \\
+2=62\end{array}$ & $14[3]+20=62$ \\
\hline 59 & $\mathbf{B}_{20} \mathrm{H}_{16}$ & $42(20)$ & $4 n-4$ & 3-cap & $C^{3}+D^{17}$ & 3,17 & $\begin{array}{c}2[17] \\
+2=36\end{array}$ & $\begin{array}{c}2[3]+4[17] \\
+2=76\end{array}$ & $20[3]+16=76$ \\
\hline 60 & $\mathrm{~B}_{16} \mathrm{H}_{20}$ & $30(16)$ & $4 n+4$ & nido & $\mathrm{C}^{-1}+\mathrm{D}^{17}$ & $-1,17$ & $\begin{array}{c}2[17] \\
+2=36 \\
\end{array}$ & $\begin{array}{c}2[-1]+4[17] \\
+2=68\end{array}$ & $16[3]+20=68$ \\
\hline 61 & $\mathrm{~B}_{15} \mathrm{H}_{23}$ & $26(15)$ & $4 n+8$ & hypho & $\mathrm{C}^{-3}+\mathrm{D}^{18}$ & $-3,18$ & $\begin{array}{r}2[18] \\
+2=38\end{array}$ & $\begin{array}{c}2[-3]+4[18] \\
+2=68\end{array}$ & $15[3]+23=68$ \\
\hline 62 & $\mathbf{B}_{20} H_{20}$ & 40(20) & $4 n+0$ & 1-cp & $\mathrm{C}^{1}+\mathrm{D}^{19}$ & 1,19 & $\begin{array}{c}2[19] \\
+2=40\end{array}$ & $\begin{array}{c}2[1]+4[19] \\
+2=80\end{array}$ & $20[3]+20=80$ \\
\hline 63 & $\mathrm{~B}_{18} \mathrm{H}_{22}$ & $34(18)$ & $4 n+4$ & nido & $\mathrm{C}^{-1}+\mathrm{D}^{19}$ & $-1,19$ & $\begin{array}{c}2[19] \\
+2=40\end{array}$ & $\begin{array}{c}2[-1]+4[19] \\
+2=76\end{array}$ & $18[3]+22=76$ \\
\hline 64 & $\mathrm{~B}_{20} \mathrm{H}_{22}$ & $39(20)$ & $4 n+2$ & closo & $\mathrm{C}^{0}+\mathrm{D}^{20}$ & 0,20 & $\begin{array}{r}2[20] \\
+2=42 \\
\end{array}$ & $\begin{array}{c}2[0]+4[20] \\
+2=82\end{array}$ & $20[3]+22=82$ \\
\hline 65 & $\overline{\mathbf{B}_{23} \mathrm{H}_{19}}$ & $48(23)$ & $4 n-4$ & 3-cap & $\mathrm{C}^{3}+\mathrm{D}^{20}$ & 3,20 & $\begin{array}{r}2[20] \\
+2=42\end{array}$ & $\begin{array}{c}2[3]+4[20] \\
+2=88\end{array}$ & $23[3]+19=88$ \\
\hline 66 & $\mathrm{~B}_{20} \mathrm{H}_{26}$ & $37(20)$ & $4 n+6$ & arachno & $\mathrm{C}^{-2}+\mathrm{D}^{22}$ & $-2,22$ & $\begin{array}{c}2[22] \\
+2=46\end{array}$ & $\begin{array}{c}2[-2]+4[22] \\
+2=86\end{array}$ & $20[3]+26=86$ \\
\hline
\end{tabular}




\section{Conventional Capping in Borane Clusters}

The proper capping based on $4 \mathrm{~N}$ series approach is very rare in borane clusters unlike the clusters such as those found in golden and transition metal complexes. According to the sample of clusters analyzed in this paper, only 3 were found. These are $\mathrm{B}_{20} \mathrm{H}_{16}$, and $\mathrm{K}^{*}=\mathrm{C}^{3}+\mathrm{D}^{17}$ (tri-capped) is given in Figure $66, \mathrm{~B}_{20} \mathrm{H}_{20}, \mathrm{~K}^{*}=\mathrm{C}^{1}+\mathrm{D}^{19}$ (mono-capped) Figure 67 and $\mathrm{B}_{23} \mathrm{H}_{19} ; \mathrm{K}^{*}=\mathrm{C}^{3}+\mathrm{D}^{20}$.

$\mathbf{D}^{17}$

48. $\mathrm{F}=\mathrm{B}_{20} \mathrm{H}_{16}: \mathrm{K}=20[2.5]-8=42, \mathrm{n}=20$

$\mathrm{K}(\mathrm{n})=42(20)$

$2[20]-42=-2$

$\mathrm{S}=4 \mathrm{n}-4$

$\mathrm{K}=2 \mathrm{n}+2$

$\mathrm{Kp}=\mathrm{C}^{3} \mathrm{C}[\mathrm{M} 17]$

$\mathrm{K}^{*}=\mathrm{C}^{3}+\mathrm{D}^{17}$

$\mathrm{y}=3, \mathrm{z}=17$

$\mathrm{VE} 0=2 \mathrm{z}+2=2[17]+2=36$

$\mathrm{VE}=\mathrm{VE} 0+2 \mathrm{n}=36+2[20]=76$

$\mathrm{VEDz}=4 \mathrm{z}+2=4[17]=2=70$

$\mathrm{VE}=\mathrm{VED} z+2 \mathrm{y}=70+2[3]=76$

$\mathrm{VE}=2 \mathrm{y}+4 \mathrm{z}+2=2[3] \mathrm{C}+4[17]+2=76$

$\mathrm{VE}=8 \mathrm{n}-2 \mathrm{~K}=8[20]-2[42]=76$

$\mathrm{VE}=4 \mathrm{n}-4=4[20]-4=76$

$\mathrm{VF}=20[3]+16=76$

$\mathrm{VE}=\mathrm{VE} 0+2 \mathrm{y}+2 \mathrm{z}=36+[3]+2[17]=76$

$K=2 z-1=2[17]-1=33$
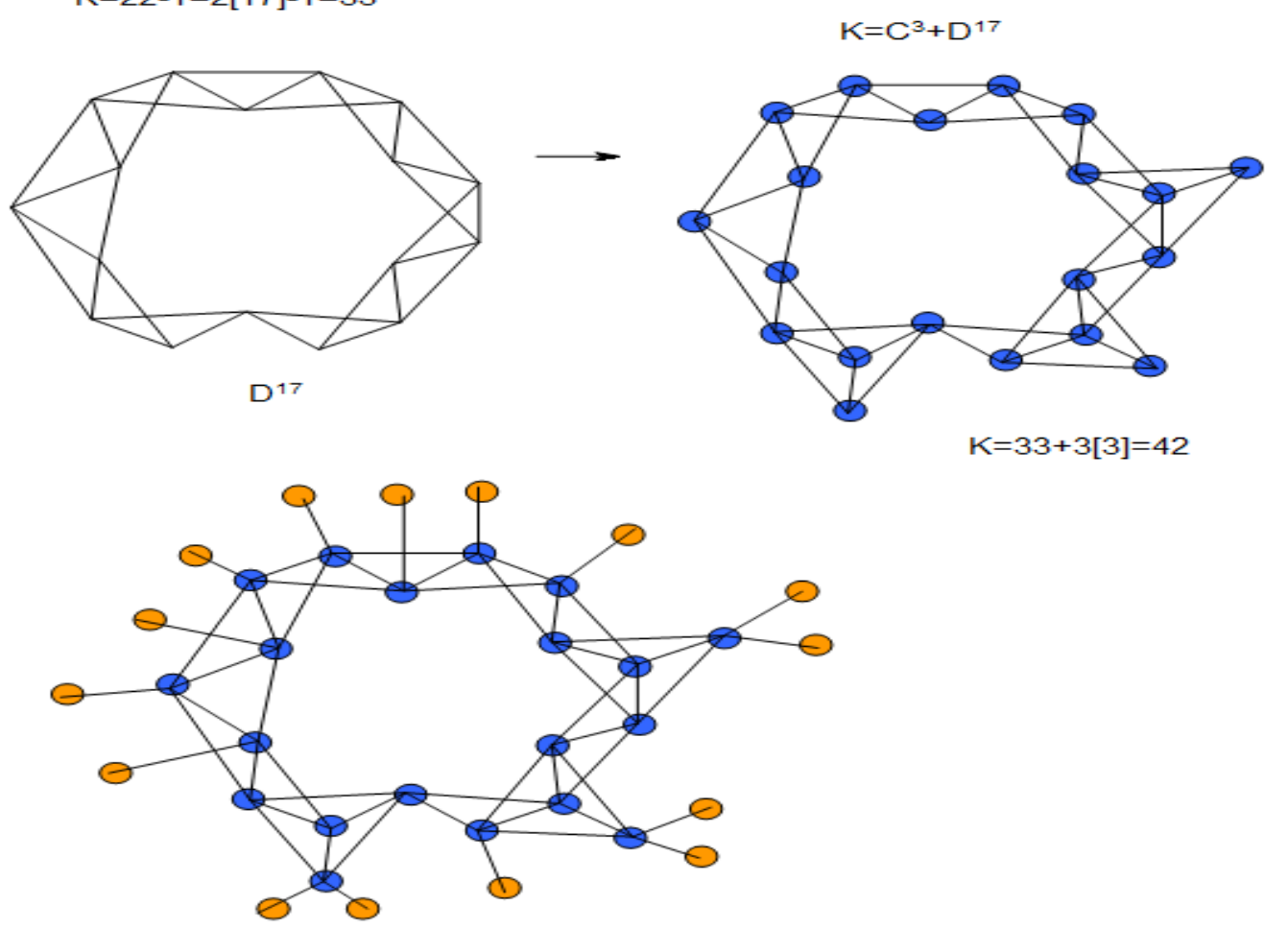

Figure 66. Isomeric graphical structure of $\mathrm{B}_{20} \mathrm{H}_{16}$ 
47. $\mathrm{B}_{20} \mathrm{H}_{20}: \mathrm{K}=20[2.5]-20[0.5]=40, \mathrm{n}=20$
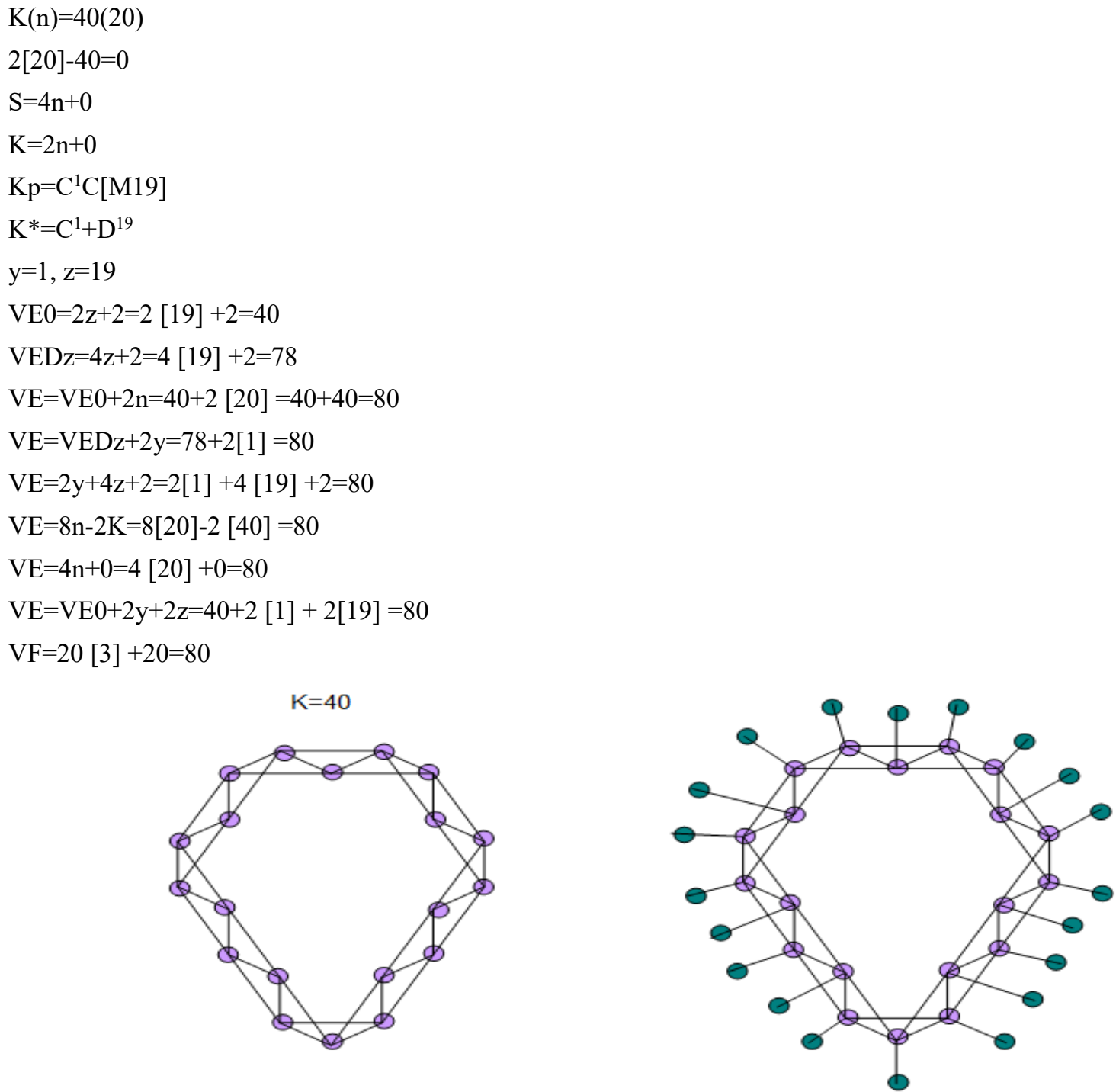

$\mathrm{B}(\mathrm{K}=2.5, \mathrm{~V}=5$

Figure 67. Isomeric graphical structure of $\mathrm{B}_{20} \mathrm{H}_{20}$

\section{Conclusion}

The borane clusters have been categorized into clan series. The six newly discovered equations for calculating cluster valence electrons have been demonstrated. The formation of triangles in borane clusters could be associated with the attainment of 5 linkage nodes at borane nodal points and the polyhedral nature of borane clusters. A few rare normal capping borane have been identified. The number of triangles of borane clusters formed using the $4 \mathrm{~N}$ approach for closo system are the same as nuclearity index of a cluster. The borane formulas follow a well-organized mathematical sequence.

\section{Acknowledgement}

The National Planning Authority, Uganda is highly acknowledged for the provision of facilities.

\section{References}

Hawthorne, M. F., Madema, A., \& Varadarajan, A. (1990). Radiometallacarboranes as tumor imaging reagents. J. Am. Chem. Soc., 112(13), 5365-5366. https://doi.org/10.1021/ja00169a062

Housecroft, C. E., \& Sharpe, A. G. (2005). Inorganic Chemistry, $2^{\text {nd }}$ Ed., Pearson, Prentice Hall, Harlow, England.

Jemmis, E. D., \& Balakrishnarajan, M. M. (2001a). Polyhedral boranes and elemental boron. Direct structural relations and diverse electronic requirements. J. Am. Chem. Soc., 123, 4324-4330. https://doi.org/10.1021/ja0026962

Jemmis, E. D., Balakrishnarajan, M. M., \& Pancharatna, P. D. (2001b). Unifying electron counting rule for 
Macropolyhedral Boranes, Metallaboranes, and Metallocenes. J. Am. Chem. Soc., 123(18), 4313-4323. https://doi.org/10.1021/ja003233z

Kiremire, E. M. (2014). Numerical sequence of Borane Series. Orient. J. Chem., 30(3), 1055-1060. https://doi.org/10.13005/ojc/300317

Kiremire, E. M. R. (2016). A Hypothetical Model for the Formation of Transition Metal Carbonyl Clusters Based Upon 4n Series Skeletal Numbers. Int. J. Chem., 8(4), 78-110. https://doi.org/10.5539/ijc.v8n4p78

Kiremire, E. M. R. (2017a). Outstanding Applications of Skeletal Numbers to Chemical Clusters. Int. J. Chem., 9(3), 2848. https://doi.org/10.5539/ijc.v9n3p28

Kiremire, E. M. R. (2017b). Boranes, Carboranes, Metalloboranes, Transition Metal Carbonyls, and Other Cluster Formulas Obey the Law of Skeletal Numbers and Their Valences. Amer. J. Chem., 7(4), 113-144.

Kiremire, E. M. R. (2017c). Numerical Characterization of Chemical Fragments, Molecules and Clusters Using Skeletal Numbers and Nuclearity Trees. American J. Chem. 7(3), 73-96.

Kiremire, E. M. R. (2018). Graph Theory of Chemical Series and Broad Categorization of Clusters. Int. J. Chem., 10(1), 17-80. https://doi.org/10.5539/ijc.v10n1p17

Kiremire, E. M. R. (2019a). Categorization of Metalloboranes Using Skeletal Numbers. Int. J. Chemistry and Research, l(1), 24-34. https://doi.org/10.18689/ijcr-1000105

Kiremire, E. M. R. (2019b). Categorization of Transition Metal Carbonyl Clusters Using Skeletal Numbers and the Six Fundamental Equations for Calculating Cluster Valence Electrons (CVE). Int. J. Chem. Res., 1(2), 35-45. https://doi.org/10.18689/ijcr-1000106

Kiremire, E. M. R. (2019c). The Double Capping Phenomenon of Chemical Clusters. Am. J. Chem., 9(2), 33-70.

Lipscomb, W. N. (1963). Boron Hydrides. W. A. Bejamin, Inc., New York

Matsui, Y., \& Taylor, R. C. (1989). Vibrational spectra and structure of heptahydrodiborate ion $\left[\mathrm{B}_{2} \mathrm{H}_{7}{ }^{-}\right]$in glycol ethers. Spectrochem. Acta, 45A(2), 299-304. https://doi.org/10.1016/0584-8539(89)80137-0

Miessler, G., Fischer, P., \& Tarr, D. (2014). Inorganic Chemistry, (5th ed.). Pearson Education, Inc., Upper Saddle River.

Mingos, D. M. P. (1972). A General Theory for Cluster and Ring Compounds of the Main Group and Transition Elements. Nature (London), Phys. Sci., 236, 99-102. https://doi.org/10.1038/physci236099a0

Mingos, D. M. P. (1984). Polyhedral Skeletal Electron Pair Approach. Acc. Chem. Res., 17(9), 311-319. https://doi.org/10.1021/ar00105a003

Rudolph, R. W. (1976). Boranes and heteroboranes: a paradigm for the electron requirements of clusters? Acc. Chem. Res., 9(12), 446-452. https://doi.org/10.1021/ar50108a004

Stock, A. (1933). The Hydrides of Boron and Silicon. New York; Cornell University Press.

Teo, B. K., Longoni, G., \& Chung, F. R. K. (1984). Applications of Topological Electron-Counting Theory to Polyhedral Metal Clusters. Inorg. Chem., 23(9), 1257-1266. https://doi.org/10.1021/ic00177a018

Wade, A. (1976). Structural and Bonding Patterns in Cluster Chemistry. Adv. Inorg. Chem. Radiochem., 18, 1-66. https://doi.org/10.1016/S0065-2792(08)60027-8

Wade, K. (1971). The structural significance of the number of skeletal bonding electron-pairs in carboranes, the higher boranes and borane ions and various transition metal carbonyl cluster compounds. Chem. Commun., 792-793. https://doi.org/10.1039/c29710000792

Welch, A. J. (2013). The significance of Wade's rules. Chem. Commun., 49, 3615-3616. https://doi.org/10.1039/c3cc00069a

\section{Copyrights}

Copyright for this article is retained by the author(s), with first publication rights granted to the journal.

This is an open-access article distributed under the terms and conditions of the Creative Commons Attribution license (http://creativecommons.org/licenses/by/4.0/). 